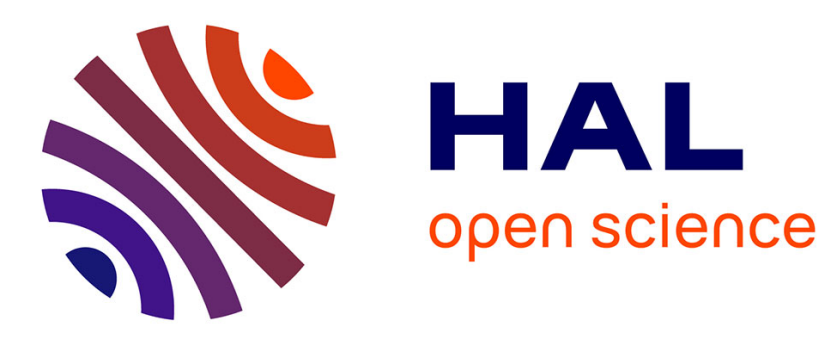

\title{
Kinetic derivation of Cahn-Hilliard fluid models Vincent Giovangigli
}

\section{To cite this version:}

Vincent Giovangigli. Kinetic derivation of Cahn-Hilliard fluid models. 2021. hal-03323739v3

\section{HAL Id: hal-03323739 \\ https://hal.science/hal-03323739v3}

Preprint submitted on 8 Nov 2021

HAL is a multi-disciplinary open access archive for the deposit and dissemination of scientific research documents, whether they are published or not. The documents may come from teaching and research institutions in France or abroad, or from public or private research centers.
L'archive ouverte pluridisciplinaire HAL, est destinée au dépôt et à la diffusion de documents scientifiques de niveau recherche, publiés ou non, émanant des établissements d'enseignement et de recherche français ou étrangers, des laboratoires publics ou privés. 


\title{
Kinetic derivation of Cahn-Hilliard fluid models
}

\author{
Vincent Giovangigli \\ CMAP-CNRS, École Polytechnique, Palaiseau, FRANCE
}

\begin{abstract}
A compressible Cahn-Hilliard fluid model is derived from the kinetic theory of dense gas mixtures. The fluid model involves a van der Waals/Cahn-Hilliard gradient energy, a generalized Korteweg's tensor, a generalized Dunn and Serrin heat flux, and Cahn-Hilliard type diffusive fluxes. Starting from the BBGKY hierarchy for gas mixtures, a Chapman-Enskog method is used-with a proper scaling of the generalized Boltzmann equations - as well as higher order Taylor expansions of pair distribution functions. An Euler/van der Waals model is obtained at zeroth order while the Cahn-Hilliard fluid model is obtained at first order involving viscous, heat and diffusive fluxes. The Cahn-Hilliard extra terms are associated with intermolecular forces and pair interaction potentials.
\end{abstract}

\section{Introduction}

Cahn-Hilliard models of fluid mixtures describe interphases - interfaces between phasesas regions with smooth variations of physical properties $[1,2,3]$. Such diffuse interface models generalize to fluid mixtures previous models developped for single species fluids $[2,3,4,5]$. Diffuse interface models have been used successfully to describe spinodal decomposition, droplets dynamics, three phase contact lines, surface diffusion, as well as transcritical flames $[1,2,3,4,5,6,7,8,9,10,11]$.

For single species fluids, the thermodynamics of diffuse interface models has been built by van der Waals [4] using a gradient squared term in the free energy and the corresponding capillary tensor has been derived by Korteweg [12]. The proper heat flux has been obtained by Dunn and Serrin in the framework of rational thermodynamics [13]. These equations have alternatively been obtained from Hamiltonian considerations by Gavrilyuk and Shugrin [14] and deduced from the kinetic theory of dense gases by Giovangigli [15].

For fluid mixtures, Cahn and Hilliard [1] first used a mole fraction gradient squared term in the free energy and later a density gradient in order to develop a thermodynamic formalism [16]. The Cahn-Hilliard equations have then been obtained in isothermal situations without convection phenomena [1], and later coupled with Navier-Stokes type equations $[17,18]$. The general situation of nonisothermal Cahn-Hilliard fluids with Navier-Stokes type equations has next been investigated using mainly thermodynamical considerations $[2,19,20,21,22,23]$. In this work, we first rederive the Cahn-Hilliard fluid equations from rational thermodynamics for completeness and in order to serve as a comparison with the kinetic theory derivation. The model obtained from rational thermodynamics that is in agreement with the kinetic derivation is found to differ from previous models of the literature $[2,19,20,21,22,23,24]$. The capillary force terms in diffusion fluxes and the capillary-diffusive contributions in the heat flux are found to differ. We establish, however, that the resulting model essentially agrees with those obtained 
in $[2,19,20,23,24]$, while some other models are found to be unphysical. Thermodynamic methods using entropy production rates are incidentally found to be ambiguous for diffuse interface models since there are various terms in the entropy production rate that involve products of several gradients as for single species fluids [15]. Cahn-Hilliard fluid models also yield discontinuous interface models when the capillarity coefficients go to zero [2]. Such heterogeneous fluid limiting models may independently be obtained from rational thermodynamics as investigated by Kjelstrup and Bedeaux [25].

Aside from macroscopic thermodynamical arguments, it is important to investigate Cahn-Hilliard fluids at the molecular level. Statistical mechanics of equilibrium systems that are highly inhomogeneous on the scale of length of intermolecular forces has been used to investigate fluid interfaces $[26,27,28,29,3]$. The links between interface structure, stress tensor, surface tension, pair distribution functions, and intermolecular forces have been deepened and this has led to expressions for the interfacial energy, the capillary pressure tensor and the capillary coefficient [26, 27, 28, 29, 3].

Considering nonequilibrium systems, the kinetic theory of gases has been used in various contexts in order to investigate phase transitions as described in the review paper by Frezzotti and Barbante [30]. Liquid-vapor phase changes have notably been investigated by employing linearized Boltzmann equations with condensation-evaporation boundary conditions [31, 32, 33]. Vlasov-Enskog type equations have also been used to investigate spatial aspects of phase transition [34, 35, 36, 37, 38, 39, 40, 41, 42, 43, 44, 45, 46]. Detailed molecular dynamics of Lennard-Jones fluids have further been performed by Frezzotti et al. [47] and compared to capillary fluid models with a general very good agreement. Concerning the derivation from the kinetic theory of diffuse interface models for single species fluids, a notable achievement was that of Rocard who derived the capillary force acting locally in a fluid using a Taylor expansion of the pair distribution function [5, 48]. Rocard recovered the Korteweg tensor and expressed the capillary coefficient in term of the interaction potential. Piechór also investigated the links between kinetic nonlocal stresses and Korteweg's tensor using an Enskog-Vlasov equation [38]. An elegant minimum BGK-Vlasov model has also been introduced by Takata and Noguchi [40] with nonideal effects mediated through a nonlocal self-consistent force term and collisions acting as a thermal bath. A gradient type energy has been obtained in the continuum limit as well a single-species Cahn-Hilliard type equation in an isothermal framework [40]. These results have then been generalized to the situation of nonisothermal fluids [46]. Still considering single species fluids, a full derivation of nonisothermal capillary fluid equations from the kinetic theory of dense gases has been obtained by Giovangigli [15]. However, to the best of the author's knowledge, a complete derivation of Cahn-Hilliard fluid mixture models from the kinetic theory of dense gas mixtures is still missing is the object of this work. The resulting model will then be compared with those obtained from rational thermodynamics.

The kinetic theory of dense gas mixtures is based on generalized Boltzmann equations for the species one-particle distribution functions [49, 50, 51], generalizing the situation of single species fluids $[52,53,54,55,56,57,58]$. Such Boltzmann equations are obtained from the two first BBGKY hierarchy equations and involve cluster expansions as well as Bogoliubov's functional property. A key point of such generalized Boltzmann equations, allowing the derivation of Cahn-Hilliard fluids, is that the collision terms involve species one-particle distribution functions evaluated at different spatial positions.

With the aim of deriving fluid macroscopic equations from the kinetic model, we introduce a new Enskog scaling for collision operators in the generalized Boltzmann equations. We next revisit and simplify the derivation of nonideal fluid equations - still without cap- 
illary effects - with viscous, heat and diffusion fluxes as investigated by Cohen et al [50]. The new scaling clarifies the analysis of fluid equations and directly yields that the zeroth order one-particle distributions are Maxwellians. Assuming that repulsive potentials are hard, some collision integrals for dense gas mixtures are also simplified in such a way that the diffusive fluxes are directly expressed in terms of chemical potential gradients in agreement with thermodynamics. In comparison, linear system inversions have been used in previous work in order to transform fluxes expressed in terms of density gradients into fluxes expressed in terms of chemical potential gradients [50, 51]. The corresponding matrices, however, may be singular in the situation of thermodynamic instabilities. The resulting macroscopic equations are found to be that of nonideal Navier-Stokes-FourierFick fluids with density dependent transport coefficients $[49,50,51]$, generalizing the results obtained for single species fluids [52, 56, 57, 58, 54].

In order to recover diffuse interface models at the Euler level - the zeroth order of Enskog expansion - we introduce symmetrized zeroth order species pair distribution functions. Higher order Taylor expansions of pair distribution functions are then performed in the conservation equations. For the sake of simplicity, the kinetic derivation is performed in the situation where the capillary coefficients are independent of temperature. As a result, we obtain the Euler/van der Waals equations for fluid mixtures, extending previous results for single species fluids [15]. The internal energy includes density gradient terms, the pressure tensor involves a generalized Korteweg type tensor and the heat flux includes a Dunn and Serrin type contribution. The diffuse interface or capillary coefficients are also related to intermolecular forces and interaction potentials.

The Cahn-Hilliard fluid model is next obtained at the Navier-Stokes level - the first order of Enskog expansion - by using higher order expansions of pair distribution functions in the conservation equations and in the linearized Boltzmann equations. The symmetrized pair distribution functions already used at the Euler level yield all relevant terms of the Cahn-Hilliard diffusion driving forces and conservation equations at first order. The alterations arising from the differences between the Bogoliubov pair distribution functions and the symmetrized pair distribution functions are also investigated and shown to be negligible in the regime investigated. The resulting Cahn-Hilliard fluid equations then coincide with the model derived from rational thermodynamics, that is, the energy density, the zeroth order extra gradient terms in the pressure tensor and the heat flux coincide as well as the Cahn-Hilliard type fluxes and the capillary-diffusive heat fluxes. The energy conservation equations and the species diffusion driving forces differ from that of previous work but the differences with some previous models are found to be unessential. Finally, the Gibbsian entropy is found to coincide with the zeroth order kinetic entropy only in the absence of capillary terms.

The equations governing Cahn-Hilliard fluids are investigated in Section 2 using rational thermodynamics. The kinetic theory of dense gas mixtures and the generalized Boltzmann equations are described in Section 3. The derivation of nonideal multicomponent fluid equations without capillary effects is presented in Section 4. The zeroth-order Euler/van der Waals equations are obtained in Section 5 and the first-order Cahn-Hilliard equations in Section 6. A discussion of the Cahn-Hilliard equations and their derivation is finally presented in Section 7.

\section{Cahn-Hilliard fluids from rational thermodynamics}

We first derive in this section the equations governing Cahn-Hilliard fluids from rational thermodynamics. These equations involve a generalized Cahn-Hilliard/van der Waals' 
energy [4], a Korteweg's tensor [12], a Dunn and Serrin's heat flux [13], Cahn-Hilliard type diffusive fluxes, and a capillary-diffusive heat flux. We also address the ambiguity of rational themodynamics - the thermodynamics of irreversible processes - for such nonlinear problems, with the apparition of various gradients' products terms in the rate of entropy production, leading to artificial alternatives for the expression of transport fluxes.

\subsection{A Cahn-Hilliard type free energy}

The Cahn-Hilliard free energy per unit volume $\mathcal{A}$ is written in the form

$$
\mathcal{A}=\mathcal{A}^{\mathrm{u}}+\sum_{i, j \in \mathfrak{S}} \frac{1}{2} \bar{\varkappa}_{i j} \nabla \rho_{i} \cdot \nabla \rho_{j}
$$

where $\mathfrak{S}=\left\{1, \ldots, \mathrm{n}_{\mathrm{s}}\right\}$ denotes the species indexing set, $\mathrm{n}_{\mathrm{s}}$ the number of species, $\mathcal{A}^{\mathrm{u}}$ the bulk free energy per unit volume, $\rho_{i}$ the partial density of the $i$ th species, $\nabla$ the usual differential operator and $\bar{\varkappa}_{i j}, i, j \in \mathfrak{S}$, the mass based capillary coefficients that are symmetric $\bar{\varkappa}_{i j}=\bar{\varkappa}_{j i}$. The superscript ${ }^{\mathrm{u}}$ is used to denote standard or bulk phase thermodynamic properties that do not involve gradients, i.e., that are also valid for spatially uniform fluids. The free energy $\mathcal{A}^{\mathrm{u}}$ only depends on the partial densities $\rho_{1}, \ldots, \rho_{n}$ and the absolute temperature $T$ whereas the gradient squared term $\sum_{i, j \in \mathfrak{S}} \frac{1}{2} \bar{\varkappa}_{i j} \nabla \rho_{i} \cdot \nabla \rho_{j}$ in $\mathcal{A}$ represents an excess free energy of the interfacial region. Such gradient energies (2.1) have notably been considered by Cahn [16], Rowlinson and Widom [3], Falk [19], Alt and Pawlow [24], Verschueren [20], Liu et al. [23], and Heida et al. [21], and reduce to the van der Waals energy for single species fluids $[2,3,4,5]$. Energies involving mass or mole fraction gradients are also easily rewritten in the general form (2.1) using properly chosen capillary coefficients $\bar{\varkappa}_{i j}$.

Denoting by $\mathcal{S}$ the Gibbsian entropy per unit volume and $\bar{g}_{i}$ the Gibbs function per unit mass of the $i$ th species, we have the thermodynamic relation $\mathrm{d} \mathcal{A}^{\mathrm{u}}=-\mathcal{S}^{\mathrm{u}} \mathrm{d} T+$ $\sum_{i \in \mathfrak{S}} \bar{g}_{i}^{\mathrm{u}} \mathrm{d} \rho_{i}$. Differentiating (2.1), it is obtained that

$$
\begin{aligned}
\mathrm{d} \mathcal{A}=-( & \left.\mathcal{S}^{\mathrm{u}}-\sum_{i, j \in \mathfrak{S}} \frac{1}{2} \partial_{T} \bar{\varkappa}_{i j} \nabla \rho_{i} \cdot \nabla \rho_{j}\right) \mathrm{d} T \\
& +\sum_{k \in \mathfrak{S}}\left(\bar{g}_{k}^{\mathrm{u}}+\sum_{i, j \in \mathfrak{S}} \frac{1}{2} \partial_{\rho_{k}} \bar{\varkappa}_{i j} \nabla \rho_{i} \cdot \nabla \rho_{j}\right) \mathrm{d} \rho_{k}+\sum_{i \in \mathfrak{S}} \bar{\gamma}_{i} \cdot \mathrm{d} \nabla \rho_{i},
\end{aligned}
$$

where $\mathrm{d}$ denotes the differentiation operator and where the mass based vectors $\bar{\gamma}_{i}, i \in \mathfrak{S}$, are defined by

$$
\bar{\gamma}_{i}=\sum_{j \in \mathfrak{S}} \bar{\varkappa}_{i j} \nabla \rho_{j}, \quad i \in \mathfrak{S} .
$$

Using the thermodynamic relations $\partial_{T} \mathcal{A}=-\mathcal{S}$ and $\partial_{\rho_{i}} \mathcal{A}=\bar{g}_{i}, i \in \mathfrak{S}$, the identity (2.2) implies that the Gibbsian entropy $\mathcal{S}$ and the species Gibbs functions par unit mass $\bar{g}_{k}$ are given by

$$
\mathcal{S}=\mathcal{S}^{\mathrm{u}}-\sum_{i, j \in \mathfrak{S}} \frac{1}{2} \partial_{T} \bar{\varkappa}_{i j} \nabla \rho_{i} \cdot \nabla \rho_{j}, \quad \bar{g}_{k}=\bar{g}_{k}^{\mathrm{u}}+\sum_{i, j \in \mathfrak{S}} \frac{1}{2} \partial_{\rho_{k}} \bar{\varkappa}_{i j} \nabla \rho_{i} \cdot \nabla \rho_{j} .
$$

The mixture Gibbs function per unit volume $\mathcal{G}$ is then found to be

$$
\mathcal{G}=\mathcal{G}^{\mathrm{u}}+\sum_{i, j, k \in \mathfrak{S}} \frac{1}{2} \rho_{k} \partial_{\rho_{k}} \bar{\varkappa}_{i j} \nabla \rho_{i} \cdot \nabla \rho_{j}
$$


and the pressure $p=\mathcal{G}-\mathcal{A}$ reads

$$
p=p^{\mathrm{u}}+\sum_{i, j \in \mathfrak{S}} \frac{1}{2}\left(\sum_{k \in \mathfrak{S}} \rho_{k} \partial_{\rho_{k}} \bar{\varkappa}_{i j}-\bar{\varkappa}_{i j}\right) \nabla \rho_{i} \cdot \nabla \rho_{j} .
$$

The equality of the Gibbsian entropy $\mathcal{S}$ with the bulk entropy $\mathcal{S}^{\mathrm{u}}$ when capillarity coefficients are independent of temperature is in agreement with van der Waals derivation in the situation of single species fluids $[4,3]$. We also note that when capillarity coefficients only depend on temperature, as will be obtained from the kinetic theory, there are no capillary contributions in the species Gibbs functions $\bar{g}_{k}=\bar{g}_{k}^{\mathrm{u}}, k \in \mathfrak{S}$. Denoting by $\mathcal{E}$ the energy per unit volume and using the thermodynamic relation $\mathcal{E}=\mathcal{A}+T \mathcal{S}$, it is further obtained that

$$
\mathcal{E}=\mathcal{E}^{\mathrm{u}}+\sum_{i, j \in \mathfrak{S}} \frac{1}{2}\left(\bar{\varkappa}_{i j}-T \partial_{T} \bar{\varkappa}_{i j}\right) \nabla \rho_{i} \cdot \nabla \rho_{j}
$$

The generalized volumetric Gibbs relation is finally obtained in the form

$$
T \mathrm{~d} \mathcal{S}=\mathrm{d} \mathcal{E}-\sum_{i \in \mathfrak{S}} \bar{g}_{i} \mathrm{~d} \rho_{i}-\sum_{i \in \mathfrak{S}} \bar{\gamma}_{i} \cdot \mathrm{d} \nabla \rho_{i}
$$

and involves the extra gradient variables $\nabla \rho_{i}, i \in \mathfrak{S}$.

\subsection{Entropy production and fluid equations}

The conservation equations for the partial densities $\rho_{i}, i \in \mathfrak{S}$, are in the form

$$
\partial_{t} \rho_{i}+\nabla \cdot\left(\rho_{i} \boldsymbol{v}\right)+\nabla \cdot \overline{\mathcal{F}}_{i}=0, \quad i \in \mathfrak{S},
$$

where $\boldsymbol{v}$ denotes the fluid velocity and $\overline{\mathcal{F}}_{i}$ the mass flux of the $i$ th species. For the sake of simplicity, no chemical source terms have been considered in the species equations. The diffusive fluxes $\overline{\mathcal{F}}_{i}, i \in \mathfrak{S}$, satisfy the mass conservation constraints $\sum_{i \in \mathfrak{S}} \overline{\mathcal{F}}_{i}=0$ so that summing the $\mathrm{n}_{\mathrm{s}}$ species conservation equations (2.9) yields the total mass conservation equation $\partial_{t} \rho+\nabla \cdot(\rho \boldsymbol{v})=0$.

The momentum conservation equation reads

$$
\partial_{t}(\rho \boldsymbol{v})+\nabla \cdot(\rho \boldsymbol{v} \otimes \boldsymbol{v})+\boldsymbol{\nabla} \cdot \mathcal{P}=0,
$$

where $\otimes$ is the tensor product between vectors and $\mathcal{P}$ the total pressure tensor, and the total energy conservation equation is in the form

$$
\partial_{t}\left(\mathcal{E}+\frac{1}{2} \rho|\boldsymbol{v}|^{2}\right)+\nabla \cdot\left(\boldsymbol{v}\left(\mathcal{E}+\frac{1}{2} \rho|\boldsymbol{v}|^{2}\right)\right)+\boldsymbol{\nabla} \cdot(\mathcal{Q}+\mathcal{P} \cdot \boldsymbol{v})=0
$$

where $\mathcal{Q}$ denotes the total heat flux. Multiplying the momentum conservation equation (2.10) by the velocity vector $\boldsymbol{v}$ and subtracting the result from the total energy conservation equation (2.11) also yields a conservation equation for the internal energy $\mathcal{E}$ as in standard fluids.

Combining the conservation equations and Gibbs relation (2.8), the following expres- 
sion for the rate of entropy production (A.7) may be obtained as detailed in Appendix A

$$
\begin{aligned}
\partial_{t} \mathcal{S}+\boldsymbol{\nabla} \cdot(\boldsymbol{v} \mathcal{S})+ & \boldsymbol{\nabla} \cdot\left(\frac{\mathcal{Q}}{T}-\sum_{i \in \mathfrak{S}} \frac{\bar{\gamma}_{i}}{T}\left(\rho_{i} \boldsymbol{\nabla} \cdot \boldsymbol{v}+\boldsymbol{\nabla} \cdot \overline{\mathcal{F}}_{i}\right)-\sum_{i \in \mathfrak{S}}\left(\frac{\bar{g}_{i}}{T}-\frac{\boldsymbol{\nabla} \cdot \overline{\boldsymbol{\gamma}}_{i}}{T}\right) \overline{\mathcal{F}}_{i}\right) \\
= & -\frac{1}{T}\left(\mathcal{P}-p \boldsymbol{I}-\sum_{i \in \mathfrak{S}}\left(\boldsymbol{\nabla} \rho_{i} \otimes \overline{\boldsymbol{\gamma}}_{i}-\rho_{i} \boldsymbol{\nabla} \cdot \overline{\boldsymbol{\gamma}}_{i} \boldsymbol{I}\right)\right): \boldsymbol{\nabla} \boldsymbol{v} \\
& -\left(\mathcal{Q}-\sum_{i \in \mathfrak{S}} \bar{\gamma}_{i}\left(\rho_{i} \boldsymbol{\nabla} \cdot \boldsymbol{v}+\boldsymbol{\nabla} \cdot \overline{\mathcal{F}}_{i}\right)+\sum_{i \in \mathfrak{S}} \boldsymbol{\nabla} \cdot \overline{\boldsymbol{\gamma}}_{i} \overline{\mathcal{F}}_{i}\right) \cdot \boldsymbol{\nabla}\left(\frac{-1}{T}\right) \\
& -\sum_{i \in \mathfrak{S}} \overline{\mathcal{F}}_{i} \cdot\left(\nabla \frac{\bar{g}_{i}}{T}-\frac{\boldsymbol{\nabla}\left(\boldsymbol{\nabla} \cdot \overline{\boldsymbol{\gamma}}_{i}\right)}{T}\right),
\end{aligned}
$$

where $\boldsymbol{I}$ denotes the unit tensor. Proceeding as in the thermodynamics of irreversible processes [59] and using the Curie principle, we deduce from the expression of entropy production (2.12) that the pressure tensor and the total heat flux are in the form

$$
\begin{aligned}
& \mathcal{P}=p \boldsymbol{I}+\sum_{i \in \mathfrak{S}} \nabla \rho_{i} \otimes \bar{\gamma}_{i}-\sum_{i \in \mathfrak{S}} \rho_{i} \boldsymbol{\nabla} \cdot \bar{\gamma}_{i} \boldsymbol{I}+\mathcal{P}^{\mathrm{d}} \\
& \mathcal{Q}=\sum_{i \in \mathfrak{S}} \bar{\gamma}_{i} \rho_{i} \boldsymbol{\nabla} \cdot \boldsymbol{v}+\sum_{i \in \mathfrak{S}} \bar{\gamma}_{i} \boldsymbol{\nabla} \cdot \overline{\mathcal{F}}_{i}-\sum_{i \in \mathfrak{S}} \boldsymbol{\nabla} \cdot \bar{\gamma}_{i} \overline{\mathcal{F}}_{i}+\mathcal{Q}^{\mathrm{d}},
\end{aligned}
$$

where $\mathcal{P}^{\mathrm{d}}$ is the viscous tensor and $\mathcal{Q}^{\mathrm{d}}$ the dissipative heat flux. The diffusion fluxes reduce to their dissipative part $\overline{\mathcal{F}}_{i}=\overline{\mathcal{F}}^{\mathrm{d}}, i \in \mathfrak{S}$ and the vectors $\bar{\gamma}_{i}, i \in \mathfrak{S}$, are given by (2.3). We also deduce that the dissipative fluxes $\mathcal{P}^{\mathrm{d}}, \overline{\mathcal{F}}_{i}, i \in \mathfrak{S}$, and $\mathcal{Q}^{\mathrm{d}}$ are in the form

$$
\begin{aligned}
\mathcal{P}^{\mathrm{d}} & =-\mathfrak{v} \boldsymbol{\nabla} \cdot \boldsymbol{v} \boldsymbol{I}-\eta\left(\boldsymbol{\nabla} \boldsymbol{v}+\boldsymbol{\nabla} \boldsymbol{v}^{t}-\frac{2}{3} \boldsymbol{\nabla} \cdot \boldsymbol{v} \boldsymbol{I}\right), \\
\overline{\mathcal{F}}_{i} & =-\sum_{j \in \mathfrak{S}} L_{i j}\left(\boldsymbol{\nabla}\left(\frac{\bar{g}_{j}}{T}\right)-\frac{\boldsymbol{\nabla} \boldsymbol{\nabla} \cdot \bar{\gamma}_{i}}{T}\right)-L_{i \mathrm{e}} \boldsymbol{\nabla}\left(\frac{-1}{T}\right), \\
\mathcal{Q}^{\mathrm{d}} & =-\sum_{i \in \mathfrak{S}} L_{\mathrm{e} i}\left(\boldsymbol{\nabla}\left(\frac{\bar{g}_{j}}{T}\right)-\frac{\boldsymbol{\nabla} \boldsymbol{\nabla} \cdot \bar{\gamma}_{i}}{T}\right)-L_{\mathrm{ee}} \boldsymbol{\nabla}\left(\frac{-1}{T}\right),
\end{aligned}
$$

where $\mathfrak{v}$ denotes the volume viscosity, $\eta$ the shear viscosity and $L_{i j}, i, j \in \mathfrak{S} \cup\{\mathrm{e}\}$, the mass and heat transport coefficients. The matrix of mass and heat transport coefficients $L$ defined by $L=\left(L_{i j}\right)_{i, j \in \mathfrak{S} \cup\{\mathrm{e}\}}$ is symmetric positive semi-definite with a nullspace spanned by the vector $(1, \ldots, 1,0)^{t}$ as for ordinary fluids $[59,60]$. The fluxes $\overline{\mathcal{F}}_{i}=\overline{\mathcal{F}}_{i}^{\mathrm{d}}, i \in \mathfrak{S}$, and $\mathcal{Q}^{\mathrm{d}}$ are obtained with (2.16) and (2.17) in their thermodynamic form $[59,61,60]$ and the link with the usual form is discussed in Section 4.4.

In the following, we will denote for convenience by $\mathcal{P}^{\mathrm{KO}}$ the Korteweg type tensor, i.e., the zeroth order gradient part of the pressure tensor $\mathcal{P}$, and by $\mathcal{Q}^{\mathrm{DS}}$ the Dunn and Serrin type heat flux, i.e., the zeroth order gradient part of the total heat flux $\mathcal{Q}$, given by

$$
\begin{aligned}
\mathcal{P}^{\mathrm{KO}}= & \sum_{i, j \in \mathfrak{S}} \frac{1}{2}\left(\sum_{k \in \mathfrak{S}} \rho_{k} \partial_{\rho_{k}} \bar{\varkappa}_{i j}-\bar{\varkappa}_{i j}\right) \nabla \rho_{i} \cdot \nabla \rho_{j} \boldsymbol{I} \\
& \quad+\sum_{i, j \in \mathfrak{S}} \bar{\varkappa}_{i j} \nabla \rho_{i} \otimes \nabla \rho_{j}-\sum_{i, j \in \mathfrak{S}} \rho_{i} \boldsymbol{\nabla} \cdot\left(\bar{\varkappa}_{i j} \nabla \rho_{j}\right) \boldsymbol{I}, \\
\mathcal{Q}^{\mathrm{DS}}= & \sum_{i, j \in \mathfrak{S}} \bar{\varkappa}_{i j} \rho_{i} \nabla \rho_{j} \boldsymbol{\nabla} \cdot \boldsymbol{v} .
\end{aligned}
$$


We will also denote by $\mathcal{Q}^{\mathrm{CD}}$ the extra capillary-diffusive first-order term given by

$$
\mathcal{Q}^{\mathrm{CD}}=-\sum_{i \in \mathfrak{S}} \nabla \cdot \bar{\gamma}_{i} \overline{\mathcal{F}}_{i}+\sum_{i \in \mathfrak{S}} \bar{\gamma}_{i} \nabla \cdot \overline{\mathcal{F}}_{i}
$$

These fluxes $\mathcal{P}^{\mathrm{KO}}, \mathcal{Q}^{\mathrm{DS}}$, and $\mathcal{Q}^{\mathrm{CD}}$ will have to be recovered from the kinetic theory as well as the expression of the dissipative fluxes. Some simplifications may further be obtained in the situation of free energies expressed in terms of mass or mole fraction gradients as discussed in Appendix B.

The mass and heat transport coefficients $L_{i j}, i, j \in \mathfrak{S} \cup\{\mathrm{e}\}$, may also be rescaled in the form

$$
\mathcal{L}_{i j}=\frac{L_{i j}}{T \rho_{i} \rho_{j}}, \quad i, j \in \mathfrak{S}, \quad \mathcal{L}_{\mathrm{e} i}=\mathcal{L}_{i \mathrm{e}}=\frac{\widehat{L}_{i \mathrm{e}}}{T \rho_{i}} \quad i \in \mathfrak{S}, \quad \mathcal{L}_{\mathrm{ee}}=\frac{\widehat{L}_{\mathrm{ee}}}{T^{2}}
$$

and we define for convenience the force term $\boldsymbol{X}_{i}$ as the opposite of the force on the $i$ th species per unit volume

$$
\boldsymbol{X}_{i}=\rho_{i} T\left(\nabla\left(\frac{\bar{g}_{i}}{T}\right)-\frac{\nabla \nabla \cdot \bar{\gamma}_{i}}{T}\right), \quad i \in \mathfrak{S} .
$$

This force term may be split as $\boldsymbol{X}_{i}=\boldsymbol{X}_{i}^{\text {th }}+\boldsymbol{X}_{i}^{\text {ch }}$ where $\boldsymbol{X}_{i}^{\text {th }}$ and $\boldsymbol{X}_{i}^{\text {ch }}$ are the opposite of the thermodynamic force and the capillary force on the $i$ th species per unit volume

$$
\boldsymbol{X}_{i}^{\text {th }}=\rho_{i} T \nabla\left(\frac{\bar{g}_{i}}{T}\right), \quad \boldsymbol{X}_{i}^{\text {ch }}=-\rho_{i} \nabla \nabla \cdot \bar{\gamma}_{i}, \quad i \in \mathfrak{S} .
$$

The thermodynamic force per unit mass of the $i$ th species is also given by $-T \nabla\left(\bar{g}_{i} / T\right)$ [59] and the capillary force per unit mass by $\nabla \nabla \cdot \bar{\gamma}_{i}$. Defining then the species diffusion velocities $\mathcal{V}_{i}, i \in \mathfrak{S}$, by $[56,57]$

$$
\mathcal{V}_{i}=\frac{\mathcal{F}_{i}}{\rho_{i}}, \quad, i \in \mathfrak{S},
$$

we may rewrite the species diffusion velocities and the heat flux in the form

$$
\begin{aligned}
\mathcal{V}_{i} & =-\sum_{j \in \mathfrak{S}} \mathcal{L}_{i j} \boldsymbol{X}_{j}-\mathcal{L}_{i \mathrm{e}} \boldsymbol{\nabla} \log T \\
\mathcal{Q}^{\mathrm{d}} & =-\sum_{i \in \mathfrak{S}} \mathcal{L}_{\mathrm{e} i} \boldsymbol{X}_{i}-\mathcal{L}_{\mathrm{ee}} \boldsymbol{\nabla} T
\end{aligned}
$$

One may also introduce the diffusion driving forces $\boldsymbol{d}_{i}=\boldsymbol{X}_{i} / n k_{\mathrm{B}} T, i \in \mathfrak{S}$, where $n$ is the total number density and $k_{\mathrm{B}}$ the Boltzmann constant. The diffusion velocities are easily rewritten in terms of such diffusion driving forces that may also be split between the thermodynamic diffusion driving forces $\boldsymbol{d}_{i}^{\text {th }}=\boldsymbol{X}_{i}^{\text {th }} / n k_{\mathrm{B}} T$ and the Cahn-Hilliard diffusion driving forces $\boldsymbol{d}_{i}^{\mathrm{ch}}=\boldsymbol{X}_{i}^{\mathrm{ch}} / n k_{\mathrm{B}} T$. Finally, in order to relate the thermodynamic form of dissipative fluxes $(2.16)(2.17)$ or $(2.25)(2.26)$ to more traditional forms, it is necessary to subtract a temperature gradient from the thermodynamic force terms $\boldsymbol{X}_{i}^{\text {th }}, i \in \mathfrak{S}$. This generally involves pressure based thermodynamic functions that explode at mechanical unstable states [9] as addressed in Section 4.5.2. 


\subsection{Ambiguity of rational thermodynamics}

An important difficulty associated with rational thermodynamics when applied to phase field models is the presence in the rate of entropy production of quantities involving products of several gradients. More specifically, in the expression (2.12) of entropy production, all terms in the form

$$
\rho_{i} \boldsymbol{\nabla} \cdot \boldsymbol{v} \bar{\gamma}_{i} \cdot \boldsymbol{\nabla}\left(\frac{-1}{T}\right), \quad i \in \mathfrak{S},
$$

involve both temperature and velocity gradients. Considering these terms as temperature gradient terms one recovers the Korteweg's type tensor $\mathcal{P}^{\mathrm{KO}}$ and Dunn and Serrin's type heat flux $\mathcal{Q}^{\text {DS }}$. However, using $\boldsymbol{\nabla} \cdot \boldsymbol{v}=\boldsymbol{\nabla} \boldsymbol{v}: \boldsymbol{I}$, these gradients' product terms (2.27) may also be considered as velocity derivative terms. This then leads to an alternative form of entropy production as well as to unphysical transport fluxes as already established in the situation of a single species fluid [15].

Similarly, various terms may be regrouped differently in the expression of the entropy production rate, as notably $\overline{\mathcal{F}}_{i} \cdot \boldsymbol{\nabla}\left(\boldsymbol{\nabla} \cdot \bar{\gamma}_{i}\right) / T, i \in \mathfrak{S}$. More specifically, the variant entropy production rate (A.6) has been used in the literature $[2,20,23]$ as well as the other variant (A.8) in [19, 24]. The resulting flux expressions only differ in the first order capillary flux $\mathcal{Q}^{\mathrm{CD}}$ and in the force terms $\boldsymbol{X}_{i}, i \in \mathfrak{S}$, all the other terms being unchanged. The first variant (A.6) leads to the flux

$$
\mathcal{Q}^{\mathrm{CD}}=\sum_{i \in \mathfrak{S}} \bar{\gamma}_{i} \nabla \cdot \overline{\mathcal{F}}_{i}
$$

with the terms $-\sum_{i \in \mathfrak{S}} \nabla \cdot \bar{\gamma}_{i} \overline{\mathcal{F}}_{i}$ missing, and to the force terms

$$
\boldsymbol{X}_{i}^{\prime}=\rho_{i} T\left(\nabla \frac{\bar{g}_{i}}{T}-\nabla \frac{\nabla \cdot \bar{\gamma}_{i}}{T}\right)=\rho_{i} T \nabla\left(\frac{\bar{g}_{i}}{T}-\frac{\nabla \cdot \bar{\gamma}_{i}}{T}\right), \quad i \in \mathfrak{S},
$$

with the factor $1 / T$ inside the gradient operator. The other variant (A.8) leads to the heat flux

$$
\mathcal{Q}^{\prime \prime \mathrm{CD}}=0,
$$

with both terms $-\sum_{i \in \mathfrak{S}} \nabla \cdot \bar{\gamma}_{i} \overline{\mathcal{F}}_{i}$ and $\sum_{i \in \mathfrak{S}} \bar{\gamma}_{i} \boldsymbol{\nabla} \cdot \overline{\mathcal{F}}_{i}$ missing, and to the force terms

$$
\boldsymbol{X}_{i}^{\prime \prime}=\rho_{i} T\left(\nabla \frac{\bar{g}_{i}}{T}-\nabla \nabla \cdot \frac{\bar{\gamma}_{i}}{T}\right)=\rho_{i} T \nabla\left(\frac{\bar{g}_{i}}{T}-\nabla \cdot \frac{\bar{\gamma}_{i}}{T}\right), \quad i \in \mathfrak{S},
$$

with the factor $1 / T$ inside the gradient and divergence operators. Even though these fluxes differ from the results obtained from the kinetic theory, we will see in Section 7.1 that these models essentially agree since temperature variations and diffusive fluxes are modest in interphase fronts.

Finally, the other variant (A.9) leads to the flux

$$
\mathcal{Q}^{\prime \prime C D}=-\sum_{i \in \mathfrak{S}} \bar{g}_{i} \overline{\mathcal{F}}_{i}-\sum_{i \in \mathfrak{S}} \nabla \cdot \bar{\gamma}_{i} \overline{\mathcal{F}}_{i}+\sum_{i \in \mathfrak{S}} \bar{\gamma}_{i} \nabla \cdot \overline{\mathcal{F}}_{i}
$$

that contains the unphysical term $-\sum_{i \in \mathfrak{S}} \bar{g}_{i} \overline{\mathcal{F}}_{i}$. Even in the absence of capillarity terms, the heat flux $\mathcal{Q}=\mathcal{Q}^{\mathrm{d}}-\sum_{i \in \mathfrak{S}} \bar{g}_{i} \overline{\mathcal{F}}_{i}$ is in contradiction with classical models of fluid mixtures $[59,62,63,64,60]$.

Rational thermodynamics, that is usually a faithful tool for linear-like problems, is here unable to determine unambiguously the proper transport fluxes. For such nonlinear problems, there is a failure of rational thermodynamics in the sense that simple algebraic reorderings of the entropy production rate seem to lead to different physical fluxes and thus to different physics. This is why only finer physical theories, like the kinetic theory of dense gas mixtures, may determine unambiguously the proper fluxes and equations. 


\subsection{Equilibrium interfaces}

The structure of equilibrium phase interfaces at zero velocity may be obtained extremalizing the entropy $\mathcal{S}$ for given amounts of species mass $\rho_{k}, k \in \mathfrak{S}$, and energy $\mathcal{E}[65,9]$.

Using the method of Lagrange multipliers, the infinitesimal variation of interfacial entropy reads

$$
\delta \int\left(\mathcal{S}-\alpha_{e} \mathcal{E}-\sum_{k \in \mathfrak{S}} \alpha_{k} \rho_{k}\right) \mathrm{d} \boldsymbol{x}=0
$$

where $\alpha_{k}, k \in \mathfrak{S}$, and $\alpha_{e}$ are Lagrange multipliers and $\delta$ is the variational symbol. Using Gibbs relation (2.8) and a few integration by parts, it is obtained from (2.33) that

$$
\int\left\{\left(\frac{1}{T}-\alpha_{e}\right) \delta \mathcal{E}+\sum_{k \in \mathfrak{S}}\left(-\frac{\bar{g}_{k}}{T}+\nabla \cdot \frac{\bar{\gamma}_{k}}{T}-\alpha_{k}\right) \delta \rho_{k}\right\} \mathrm{d} \boldsymbol{x}=0 .
$$

Since the variations $\delta \mathcal{E}$ and $\delta \rho_{k}, k \in \mathfrak{S}$, are arbitrary in (2.34), we conclude that

$$
\begin{aligned}
& \frac{1}{T}-\alpha_{e}=0 \\
& -\frac{\bar{g}_{k}}{T}+\nabla \cdot \frac{\bar{\gamma}_{k}}{T}-\alpha_{k}=0, \quad k \in \mathfrak{S} .
\end{aligned}
$$

The interface is thus isothermal and the generalized chemical potentials $\bar{g}_{k}-\nabla \cdot \bar{\gamma}_{k}$ are also constant through the interface. Taking the gradient of these relations, we obtain that $\boldsymbol{\nabla} T=0$ and that the force terms $(2.22)$ vanish $\boldsymbol{X}_{k}=0, k \in \mathfrak{S}$. As a consequence, the heat flux $\mathcal{Q}$ as well as the species mass fluxes $\overline{\mathcal{F}}_{k}, k \in \mathfrak{S}$, vanish through the interface that may also be convected at any velocity.

Assuming that capillarity coefficients $\bar{\varkappa}_{i j}, i, j \in \mathfrak{S}$, are constant for the sake of simplicity, multiplying (2.36) by $\boldsymbol{\nabla} \rho_{k}$, summing over $k \in \mathfrak{S}$, and keeping in mind that the interface is isothermal, we obtain that $\boldsymbol{\nabla} \mathcal{A}^{\mathrm{u}}-\sum_{k \in \mathfrak{S}} \nabla \rho_{k} \boldsymbol{\nabla} \cdot \bar{\gamma}_{k}+T \sum_{k \in \mathfrak{S}} \nabla \rho_{k} \alpha_{k}=0$. Multiplying (2.36) by $\rho_{k}$, summing over $k \in \mathfrak{S}$, we also deduce that $\mathcal{G}^{\mathrm{u}}-\sum_{k \in \mathfrak{S}} \rho_{k} \boldsymbol{\nabla}$. $\bar{\gamma}_{k}+T \sum_{k \in \mathfrak{S}} \rho_{k} \alpha_{k}=0$. Taking the gradient of this identity and subtracting the former relation then yields that $\nabla p^{\mathrm{u}}-\sum_{k \in \mathfrak{S}} \rho_{k} \boldsymbol{\nabla} \nabla \cdot \bar{\gamma}_{k}=0$ that is the mechanical equilibrium condition. Such a structure of equilibrium fronts shows that, in an interphase, typically a vaporization front, density gradients may be very large, but we may assume that temperature variations as well as diffusion fluxes remain modest in a macroscopic regime. Considering also two phases at equilibrium denoted with the superscript $\sharp$ and ${ }^{b}$, and letting the gradients go to zero far from the interface, we obtain the classical equilibrium conditions $T^{\sharp}=T^{b}, \bar{g}_{k}^{\sharp}=\bar{g}_{k}^{b}, k \in \mathfrak{S}$, and $p^{\sharp}=p^{b}$ between the phases.

In the situation of single species fluids, we may further investigate the structure of a one-dimensional vaporization front $[4,3,6]$. Denoting by $l$ and $g$ the indices of the liquid and its vapor at equilibrium, the equilibrium relations yields $T_{1}=T_{\mathrm{g}}, p_{1}=p_{\mathrm{g}}$, and $\bar{g}_{1}=\bar{g}_{\mathrm{g}}$. Denoting by $z$ the normal coordinate, the isothermal interface is such that $\frac{1}{2} \varkappa(\mathrm{d} \rho / \mathrm{d} z)^{2}=\mathcal{A}-\mathcal{A}_{\mathrm{g}}-\bar{g}_{\mathrm{g}}\left(\rho-\rho_{\mathrm{g}}\right)$. The function $\mathcal{A}-\mathcal{A}_{\mathrm{g}}-\bar{g}_{\mathrm{g}}\left(\rho-\rho_{\mathrm{g}}\right)$ is the difference between $\mathcal{A}$ and its bi-tangent line, since $\mathcal{A}_{\mathrm{l}}=\mathcal{A}_{\mathrm{g}}-\bar{g}_{\mathrm{g}}\left(\rho_{\mathrm{l}}-\rho_{\mathrm{g}}\right)$ from the equality of pressures and since $\partial_{\rho} \mathcal{A}=\bar{g}$ at constant temperature. Approximating the excess function $\mathcal{A}-\mathcal{A}_{\mathrm{g}}-\bar{g}_{\mathrm{g}}\left(\rho-\rho_{\mathrm{g}}\right)$ as $\overline{\mathcal{A}}\left(\rho-\rho_{\mathrm{l}}\right)^{2}\left(\rho-\rho_{\mathrm{g}}\right)^{2}$, where $\overline{\mathcal{A}}$ is a constant, the density is found in the form $\rho(z)=\frac{1}{2}\left(\rho_{\mathrm{l}}+\rho_{\mathrm{g}}\right)+\frac{1}{2}\left(\rho_{\mathrm{l}}-\rho_{\mathrm{g}}\right) \tanh (z / 2 \bar{z})$ with the characteristic length $\bar{z}=(\varkappa / 2 \overline{\mathcal{A}})^{1 / 2} /\left(\rho_{\mathrm{l}}-\rho_{\mathrm{g}}\right)$. 


\subsection{Terminology}

The traditional terminology used for fluids involving gradient terms in free energies is not without criticisms. The word capillary is traditionally used to denote the extra gradient terms in the energy, the pressure tensor, and the heat or mass fluxes. Historically, this terminology is associated with fluid rising in thin tubes or capillaries. However, there are not necessary thin tubes in a given fluid and discontinous multiphase models also imply the rise of liquids in thin tubes provided Laplace's law is used and three point boundary conditions are applied at contact lines. In other words, the rise of fluids in thin tubes is due to curvature effects, Laplace's law, and to the interaction with tube walls [3], instead of being a property of the bulk fluid.

A much better terminology is that of diffuse interface. The higher order derivative terms indeed lead to transition zones between phases where thermodynamic and fluid properties are smooth, thereby the terminology diffuse. Such terms are also mainly important in thin transition zones between phases and thus the vocable interface is also an excellent denomination. The gradient energy terms, that are due to potential interaction between particles, are also present in supercritical fluids where in principle, strictly speaking, there are no interfaces, but where one may still find sharp transition zones [9].

Another very good terminology is that of cohesive fluids. All the gradient terms are indeed due to cohesive forces that are also independent of the state of the fluid. This terminology is often used with cohesion of a fluid or cohesive forces and could also be used for such capillary or diffuse interface fluids. The only possible ambiguity is that attractive terms in van der Waals type equations are also sometimes termed cohesion coefficients. In this work, we will equivalently use capillary, diffuse interface or cohesive.

\section{$3 \quad$ A dense gas mixture kinetic model}

A kinetic theory describing moderately dense gas mixtures is summarized in this section as well as the corresponding macroscopic fluid equations [50].

\subsection{The BBGKY hierarchy}

We consider a dense gas mixture and the associated BBGKY hierarchy-also termed the YBG hierarchy [3] - governing multiple-particle distribution functions [50]. It is assumed, for the sake of simplicity, that there are no external forces acting on the particles, that the species are monatomic, and that no chemical reactions are involved between the species. The two first equations of the BBGKY hierarchy for fluid mixtures are then in the form

$$
\begin{aligned}
& \partial_{t} f_{i}+\boldsymbol{c}_{i} \cdot \nabla_{\mathbf{r}_{i}} f_{i}=\sum_{j \in \mathfrak{S}} \int \theta_{i j} f_{i j} \mathrm{~d} \boldsymbol{x}_{j}, \\
& \partial_{t} f_{i j}+\boldsymbol{c}_{i} \cdot \nabla_{\mathbf{r}_{i}} f_{i j}+\boldsymbol{c}_{j} \cdot \nabla_{\mathbf{r}_{j}} f_{i j}-\theta_{i j} f_{i j}=\sum_{k \in \mathfrak{S}} \int\left(\theta_{i k}+\theta_{j k}\right) f_{i j k} \mathrm{~d} \boldsymbol{x}_{k},
\end{aligned}
$$

where $\partial_{t}$ denotes the time derivative operator, $f_{i}$ the one-particle distribution of the $i$ th species, $\mathbf{r}_{i}$ the spatial position of a particle of the $i$ th species, $\boldsymbol{c}_{i}$ the velocity of a particle of the $i$ th species, $f_{i j}$ the pair distribution of the $(i, j)$ th pair of species, $f_{i j k}$ the triplet distribution of the $(i, j, k)$ th triplet of species, $\boldsymbol{\nabla}_{\mathbf{r}_{i}}$ the derivative operator with respect to the spatial coordinate $\mathbf{r}_{i}$, and $\boldsymbol{\nabla}_{\boldsymbol{c}_{i}}$ the derivative operator with respect to the velocity 
$\boldsymbol{c}_{i}$. We also denote for short by $\boldsymbol{x}_{i}$ the pair of vectors $\boldsymbol{x}_{i}=\left(\mathbf{r}_{i}, \boldsymbol{c}_{i}\right)$ and by d $\boldsymbol{x}_{i}$ the volume element $\mathrm{d} \mathbf{r}_{i} \mathrm{~d} \boldsymbol{c}_{i}$. The differential operator $\theta_{i j}$ is given by

$$
\theta_{i j}=\frac{1}{m_{i}} \nabla_{\mathbf{r}_{i}} \varphi_{i j} \cdot \nabla_{\boldsymbol{c}_{i}}+\frac{1}{m_{j}} \nabla_{\mathbf{r}_{j}} \varphi_{i j} \cdot \nabla_{\boldsymbol{c}_{j}}
$$

where $m_{i}$ denotes the mass of a particle of the $i$ th species and $\varphi_{i j}$ the interaction potential between particles of the $i$ th and $j$ th species that only depends on the interparticle distance $r_{i j}=\left|\mathbf{r}_{j}-\mathbf{r}_{i}\right|$ and we denote by $\mathbf{r}_{i j}=\mathbf{r}_{j}-\mathbf{r}_{i}$ the difference between the particle spatial positions. Strictly speaking, we should denote differently the spatial positions of two colliding particles when $i=j$, but these subtleties will be left implicit for the sake of notational simplicity [50]. We will also sometimes denote by $\mathbf{r}$ a common coordinate for all distributions $f_{i}, i \in \mathfrak{S}$. The traditional distribution functions are $f_{i}=f_{i}\left(\boldsymbol{x}_{i}, t\right)=$ $f_{i}\left(\mathbf{r}_{i}, \boldsymbol{c}_{i}, t\right)$, the particle pair distribution functions are $f_{i j}=f_{i j}\left(\boldsymbol{x}_{i}, \boldsymbol{x}_{j}, t\right)$ and satisfy the symmetry relations $f_{i j}\left(\boldsymbol{x}_{i}, \boldsymbol{x}_{j}, t\right)=f_{j i}\left(\boldsymbol{x}_{j}, \boldsymbol{x}_{i}, t\right)$. The triplet particle distribution functions $f_{i j k}$ similarly depend on $\left(\boldsymbol{x}_{i}, \boldsymbol{x}_{j}, \boldsymbol{x}_{k}, t\right)$ but triple collision effects are not taken into account in this work. For a single gas, the equations of the BBGKY hierarchy have been derived independently by Bogoliubov [66], Born and Green [67], Kirkwood [68], and introduced by Yvon [69], and we refer to the literature [50, 51, 56, 57, 70] for more details.

The aim of this work being the derivation of macroscopic equations governing CahnHilliard type fluids, we only use a simplified kinetic theory of moderately dense gas mixtures. In particular internal states, triple collisions or chemical reactions are not taken into account and we will also simplify some of the collision integrals associated with dense gases. We refer to the literature for various difficulties associated with dense gas mixtures like bound states, divergences with quadruple collisions in three dimensions, long time tails for time correlation functions $[52,56,57,58,54]$ as well as for internal states and chemical reactions [62, 63, 64].

\subsection{Macroscopic equations}

The gas number density of the $i$ th species $n_{i}$ is given by

$$
n_{i}\left(\mathbf{r}_{i}, t\right)=\int f_{i}\left(\mathbf{r}_{i}, \boldsymbol{c}_{i}, t\right) \mathrm{d} \boldsymbol{c}_{i}
$$

the total number density $n$ by $n(\mathbf{r}, t)=\sum_{i \in \mathfrak{S}} n_{i}(\mathbf{r}, t)$, and the pair number densities $n_{i j}$ read

$$
n_{i j}\left(\mathbf{r}_{i}, \mathbf{r}_{j}, t\right)=\int f_{i j}\left(\mathbf{r}_{i}, \boldsymbol{c}_{i}, \mathbf{r}_{j}, \boldsymbol{c}_{j}, t\right) \mathrm{d} \boldsymbol{c}_{i} \mathrm{~d} \boldsymbol{c}_{j} .
$$

The partial density of the $i$ th species $\rho_{i}$ is given by $\rho_{i}=m_{i} n_{i}$, the total mass density by $\rho(\mathbf{r}, t)=\sum_{i \in \mathfrak{S}} \rho_{i}(\mathbf{r}, t)$ and the gas velocity $\boldsymbol{v}$ is defined by

$$
\rho(\mathbf{r}, t) \boldsymbol{v}(\mathbf{r}, t)=\sum_{i \in \mathfrak{S}} \int m_{i} \boldsymbol{c}_{i} f_{i}\left(\mathbf{r}, \boldsymbol{c}_{i}, t\right) \mathrm{d} \boldsymbol{c}_{i},
$$

where the distributions are evaluated at a common location $\mathbf{r}=\mathbf{r}_{i}, i \in \mathfrak{S}$.

The internal energy per unit volume of the fluid is decomposed into

$$
\mathcal{E}=\mathcal{E}^{\mathrm{K}}+\mathcal{E}^{\mathrm{P}}
$$

where $\mathcal{E}^{\mathrm{K}}$ is the kinetic part and originates from the peculiar motion of the particles whereas $\mathcal{E}^{\mathrm{P}}$ is the potential part due to the species pair interaction potentials $[50,56,57]$. 
The kinetic part $\mathcal{E}^{\mathrm{K}}$ and the potential part $\mathcal{E}^{\mathrm{P}}$ may be written

$$
\begin{array}{r}
\mathcal{E}^{\mathrm{K}}(\mathbf{r}, t)=\sum_{i \in \mathfrak{S}} \int \frac{1}{2} m_{i}\left|\boldsymbol{c}_{i}-\boldsymbol{v}\right|^{2} f_{i}\left(\mathbf{r}, \boldsymbol{c}_{i}, t\right) \mathrm{d} \boldsymbol{c}_{i}, \\
\mathcal{E}^{\mathrm{P}}(\mathbf{r}, t)=\sum_{i, j \in \mathfrak{S}} \int \frac{1}{2} \varphi_{i j}\left(r_{i j}\right) n_{i j}\left(\mathbf{r}, \mathbf{r}+\mathbf{r}_{i j}, t\right) \mathrm{d} \mathbf{r}_{i j},
\end{array}
$$

where we have used $\mathrm{d} \boldsymbol{x}_{j}=\mathrm{d} \mathbf{r}_{j} \mathrm{~d} \boldsymbol{c}_{j}=\mathrm{d} \mathbf{r}_{i j} \mathrm{~d} \boldsymbol{c}_{j}$.

The general macroscopic equations in unclosed form may further be obtained by taking appropriate moments of (3.1) and (3.2) as detailed in Appendix C. The mass conservation equation is derived by integrating (3.1) with respect to $\boldsymbol{c}_{i}$ and reads

$$
\partial_{t} \rho_{i}+\nabla \cdot\left(\rho_{i} \boldsymbol{v}\right)+\nabla \cdot \overline{\mathcal{F}}_{i}=0
$$

where the mass diffusion flux of the $i$ th species $\overline{\mathcal{F}}_{i}$ is given by

$$
\overline{\mathcal{F}}_{i}=\int m_{i}\left(\boldsymbol{c}_{i}-\boldsymbol{v}\right) f_{i} \mathrm{~d} \boldsymbol{c}_{i}
$$

The momentum equation, obtained by multiplying (3.1) by $m_{i} \boldsymbol{c}_{i}$, integrating with respect to $\boldsymbol{c}_{i}$, and summing over $i \in \mathfrak{S}$, is in the form

$$
\partial_{t}(\rho \boldsymbol{v})+\boldsymbol{\nabla} \cdot(\rho \boldsymbol{v} \otimes \boldsymbol{v})+\boldsymbol{\nabla} \cdot \mathcal{P}=0
$$

and the total pressure tensor $\mathcal{P}$ is decomposed into

$$
\mathcal{P}=\mathcal{P}^{\mathrm{K}}+\mathcal{P}^{\mathrm{P}}
$$

The tensor $\mathcal{P}^{\mathrm{K}}$ is the traditional kinetic part

$$
\mathcal{P}^{\mathrm{K}}(\mathbf{r}, t)=\sum_{i \in \mathfrak{S}} \int m_{i}\left(\boldsymbol{c}_{i}-\boldsymbol{v}\right) \otimes\left(\boldsymbol{c}_{i}-\boldsymbol{v}\right) f_{i}\left(\mathbf{r}, \boldsymbol{c}_{i}, t\right) \mathrm{d} \boldsymbol{c}_{i},
$$

whereas $\mathcal{P}^{\mathrm{P}}$ is the potential part

$$
\mathcal{P}^{\mathrm{P}}(\mathbf{r}, t)=-\sum_{i, j \in \mathfrak{S}} \frac{1}{2} \int \frac{\varphi_{i j}^{\prime}\left(r_{i j}\right)}{r_{i j}} \mathbf{r}_{i j} \otimes \mathbf{r}_{i j} n_{i j}\left(\mathbf{r}-(1-\alpha) \mathbf{r}_{i j}, \mathbf{r}+\alpha \mathbf{r}_{i j}, t\right) \mathrm{d} \alpha \mathrm{d} \mathbf{r}_{i j},
$$

and the scalar $\alpha$ must be integrated over $(0,1)$. The potential part of the pressure tensor $\mathcal{P}^{\mathrm{P}}(\mathbf{r}, t)$ has been obtained in a form similar to that of single species fluids [57] that is more convenient than the expressions presented by Cohen et al. [50]. The tensor $\mathcal{P}^{\mathrm{K}}$ corresponds to the transfer of momentum due to the flow of particles and $\mathcal{P}^{\mathrm{P}}$ to the transfer of momentum among particles due to intermolecular forces between species pairs $[56,57]$.

The conservation equation for internal energy $\mathcal{E}$ is next derived in several steps and may also be combined with the kinetic energy conservation equation in order to derive the total energy conservation equation. One first multiply (3.1) by $\frac{1}{2} m_{i}\left|\boldsymbol{c}_{i}-\boldsymbol{v}\right|^{2}$, integrate with respect to $\boldsymbol{c}_{i}$, and sum over $i \in \mathfrak{S}$, in order to obtain a balance equation for the kinetic part $\mathcal{E}^{\mathrm{K}}$ of the internal energy. The balance equation for the potential part of the energy $\mathcal{E}^{\mathrm{P}}$ is then obtained by multiplying the two-particle distribution function equation (3.2) by the potential energy $\frac{1}{2} \varphi_{i j}\left(r_{i j}\right)$, integrating with respect to $\boldsymbol{c}_{i}, \boldsymbol{c}_{j}$, and $\mathbf{r}_{i j}=\mathbf{r}_{j}-\mathbf{r}_{i}$, and 
summing over $i, j \in \mathfrak{S}$. After lengthy calculations, the governing equation for $\mathcal{E}=\mathcal{E}^{\mathrm{K}}+\mathcal{E}^{\mathrm{P}}$ is found in the form

$$
\partial_{t} \mathcal{E}+\nabla \cdot(\boldsymbol{v} \mathcal{E})+\nabla \cdot \mathcal{Q}=-\mathcal{P}: \nabla \boldsymbol{v}
$$

where the total heat flux is decomposed into

$$
\mathcal{Q}=\mathcal{Q}^{\mathrm{K}}+\mathcal{Q}_{1}^{\mathrm{P}}+\mathcal{Q}_{2}^{\mathrm{P}}
$$

The kinetic part $\mathcal{Q}^{\mathrm{K}}$ is in the form

$$
\mathcal{Q}^{\mathrm{K}}(\mathbf{r}, t)=\sum_{i \in \mathfrak{S}} \frac{1}{2} \int m_{i}\left|\boldsymbol{c}_{i}-\boldsymbol{v}\right|^{2}\left(\boldsymbol{c}_{i}-\boldsymbol{v}\right) f_{i}\left(\mathbf{r}, \boldsymbol{c}_{i}, t\right) \mathrm{d} \boldsymbol{c}_{i},
$$

the potential parts $\mathcal{Q}_{1}^{\text {P }}$ and $\mathcal{Q}_{2}^{\text {P }}$ are given by

$$
\begin{aligned}
\mathcal{Q}_{1}^{\mathrm{P}}(\mathbf{r}, t) & =\sum_{i, j \in \mathfrak{S}} \frac{1}{2} \int \varphi_{i j}\left(r_{i j}\right)\left(\boldsymbol{c}_{i}-\boldsymbol{v}\right) f_{i j}\left(\mathbf{r}, \boldsymbol{c}_{i}, \mathbf{r}+\mathbf{r}_{i j}, \boldsymbol{c}_{j}, t\right) \mathrm{d} \boldsymbol{c}_{i} \mathrm{~d} \mathbf{r}_{i j} \mathrm{~d} \boldsymbol{c}_{j}, \\
\mathcal{Q}_{2}^{\mathrm{P}}(\mathbf{r}, t)= & -\sum_{i, j \in \mathfrak{S}} \frac{1}{4} \int \frac{\varphi_{i j}^{\prime}\left(r_{i j}\right)}{r_{i j}} \mathbf{r}_{i j} \mathbf{r}_{i j} \cdot\left(\boldsymbol{c}_{i}-\boldsymbol{v}+\boldsymbol{c}_{j}-\boldsymbol{v}\right) \\
& \times f_{i j}\left(\mathbf{r}-(1-\alpha) \mathbf{r}_{i j}, \boldsymbol{c}_{i}, \mathbf{r}+\alpha \mathbf{r}_{i j}, \boldsymbol{c}_{j}, t\right) \mathrm{d} \alpha \mathrm{d} \boldsymbol{c}_{i} \mathrm{~d} \mathbf{r}_{i j} \mathrm{~d} \boldsymbol{c}_{j},
\end{aligned}
$$

and the scalar $\alpha$ must be integrated over $(0,1)$. The potential parts of the heat flux $\mathcal{Q}_{2}^{\text {P }}$ is written here in a form similar to that of single species fluids [57] that is more convenient than the expression presented by Cohen et al. [50]. The heat flow vector $\mathcal{Q}^{\mathrm{K}}$ is similar to that of a dilute gas and represents the transfer of thermal energy due to the flow of particles. The first potential part $\mathcal{Q}_{1}^{\mathrm{P}}$ represents the flow of potential energy carried by the particles and $\mathcal{Q}_{2}^{\text {P }}$ represents the flux of energy associated with long range forces between species pairs and thermal agitation. The scalar product of $\mathcal{Q}_{2}^{\text {P }}$ with a normal vector to a surface element represents the work per unit time done on all species particles on one side, that move with their peculiar motion, by all species particles on the other side, and both fluxes $\mathcal{Q}_{1}^{\mathrm{P}}$ and $\mathcal{Q}_{2}^{\mathrm{P}}$ only depend on the species peculiar velocities $\boldsymbol{c}_{i}-\boldsymbol{v}$ and $\boldsymbol{c}_{j}-\boldsymbol{v}$.

\subsection{Generalized Boltzmann equations}

The kinetic theory of dense gas mixtures involves generalized Boltzmann equations governing the species one-particle distribution functions $f_{i}, i \in \mathfrak{S}$. These equations are obtained by expanding multiple distribution functions with clusters of independant particles and using Bogoliubov's method $[49,50,51]$, in a similar way as for single species fluids [52, 56, 57, 58, 54].

Neglecting the contributions arising from triple distributions or more [57, 54], the pair distribution functions $f_{i j}$ are first written in the form $f_{i j}=\mathfrak{S}_{i j} f_{i} f_{j}$ where the streaming operators $\mathfrak{S}_{i j}$ read

$$
\mathfrak{S}_{i j}=\exp \left(-t \mathfrak{H}_{i j}\right) \exp \left(t \mathfrak{H}_{i}\right) \exp \left(t \mathfrak{H}_{j}\right)
$$

with $\mathfrak{H}_{i}=\boldsymbol{c}_{i} \cdot \boldsymbol{\nabla}_{\mathbf{r}_{i}}$ denoting the Hamiltonian operator of the $i$ th particle alone and

$$
\mathfrak{H}_{i j}=\boldsymbol{c}_{i} \cdot \nabla_{\mathbf{r}_{i}}+\boldsymbol{c}_{j} \cdot \nabla_{\mathbf{r}_{j}}-\theta_{i j}
$$


the Hamiltonian operator of the $(i, j)$ th pair of particles. The streaming operators $\exp \left(t \mathfrak{H}_{s}\right)$ form Abelian one parameter groups of operators and correspond to the streaming of systems of $s$ independant particle under the influence of the Hamiltonian $\mathfrak{H}_{s}$. It has been assumed that the initial distribution is a product of uncorrelated distributions $f_{i j}\left(\boldsymbol{x}_{i}, \boldsymbol{x}_{j}, 0\right)=f_{i}\left(\boldsymbol{x}_{i}, 0\right) f_{j}\left(\boldsymbol{x}_{j}, 0\right)$. A fundamental assumption in the model is that the operators $\mathfrak{S}_{i j}\left(\boldsymbol{x}_{i}, \boldsymbol{x}_{j}, t\right)$ have finite limits as $t$ is large with respect to the collision time

$$
\tau_{i j}\left(\mathbf{r}_{i}, \boldsymbol{c}_{i}, \mathbf{r}_{j}, \boldsymbol{c}_{j}\right)=\lim _{t \rightarrow \infty} \mathfrak{S}_{i j}\left(\mathbf{r}_{i}, \boldsymbol{c}_{i}, \mathbf{r}_{j}, \boldsymbol{c}_{j}, t\right),
$$

so that the pair distribution functions are finally in the form

$$
f_{i j}=\tau_{i j} f_{i} f_{j}, \quad i, j \in \mathfrak{S},
$$

and we refer for more details to Bennett and Curtiss [49], Cohen et al. [50] and Braun et al. [51] as well as to Choh and Uhlenbeck [52], Ferziger and Kaper [57], Dorfman and van Beijeren [58], and García-Colín et al. [54] for the single species situation.

The generalized Boltzmann equations governing one-particle distribution functions $f_{i}$, $i \in \mathfrak{S}$, are then in the form

$$
\partial_{t} f_{i}+\boldsymbol{c}_{i} \cdot \nabla_{\mathbf{r}_{i}} f_{i}=\mathcal{J}_{i}(f),
$$

where $f=\left(f_{i}\right)_{i \in \mathfrak{S}}$ and the $i$ th collision operator $\mathcal{J}_{i}$ reads

$$
\mathcal{J}_{i}(f)=\sum_{j \in \mathfrak{S}} \int \theta_{i j} \tau_{i j} f_{i}\left(\mathbf{r}_{i}, \boldsymbol{c}_{i}, t\right) f_{j}\left(\mathbf{r}_{j}, \boldsymbol{c}_{j}, t\right) \mathrm{d} \boldsymbol{x}_{j} .
$$

A key point in these operators is that the one-particle species distribution functions are taken at different positions $\mathbf{r}_{i}$ and $\mathbf{r}_{j}$.

The action of the operators $\tau_{i j}$ is notably discussed in $[52,53,54,57,58,71]$ and we let for convenience

$$
\begin{aligned}
\mathbf{r}_{i}^{\prime} & =\tau_{i j} \mathbf{r}_{i}, & \mathbf{r}_{j}^{\prime} & =\tau_{i j} \mathbf{r}_{j}, \\
\boldsymbol{c}_{i}^{\prime} & =\tau_{i j} \boldsymbol{c}_{i}, & \boldsymbol{c}_{j}^{\prime} & =\tau_{i j} \boldsymbol{c}_{j} .
\end{aligned}
$$

From the conservation of momentum during a collision we have

$$
m_{i} \boldsymbol{c}_{i}+m_{j} \boldsymbol{c}_{j}=m_{i} \boldsymbol{c}_{i}^{\prime}+m_{j} \boldsymbol{c}_{j}^{\prime},
$$

and from the conservation of energy

$$
\frac{1}{2} m_{i}\left|\boldsymbol{c}_{i}\right|^{2}+\frac{1}{2} m_{j}\left|\boldsymbol{c}_{j}\right|^{2}+\varphi_{i j}\left(r_{i j}\right)=\frac{1}{2} m_{i}\left|\boldsymbol{c}_{i}^{\prime}\right|^{2}+\frac{1}{2} m_{j}\left|\boldsymbol{c}_{j}^{\prime}\right|^{2} .
$$

From the relation $f_{i j}=\tau_{i j} f_{i} f_{j}$ we further obtain the Bogoliubov distribution $[66,56]$

$$
f_{i j}\left(\mathbf{r}_{i}, \boldsymbol{c}_{i}, \mathbf{r}_{j}, \boldsymbol{c}_{j}, t\right)=f_{i}\left(\mathbf{r}_{i}^{\prime}, \boldsymbol{c}_{i}^{\prime}, t\right) f_{j}\left(\mathbf{r}_{j}^{\prime}, \boldsymbol{c}_{j}^{\prime}, t\right),
$$

and we define $\mathbf{r}_{i j}=\mathbf{r}_{j}-\mathbf{r}_{i}$ and $\mathbf{r}_{i j}^{\prime}=\mathbf{r}_{j}^{\prime}-\mathbf{r}_{i}^{\prime}$. In addition, when $r_{i j}$ is large, there is no interaction between the particles, in such a way that $\tau_{i j}$ reduces to the identity operator and the pair distribution function $f_{i j}\left(\boldsymbol{x}_{i}, \boldsymbol{x}_{j}, t\right)$ is then the product of two independant distributions $f_{i}\left(\boldsymbol{x}_{i}, t\right) f_{j}\left(\boldsymbol{x}_{j}, t\right)$.

Decomposing $f_{i j}$ in the form

$$
f_{i j}=f_{i}\left(\mathbf{r}_{i}, \boldsymbol{c}_{i}^{\prime}, t\right) f_{j}\left(\mathbf{r}_{i}, \boldsymbol{c}_{j}^{\prime}, t\right)+\left(f_{i}\left(\mathbf{r}_{i}^{\prime}, \boldsymbol{c}_{i}^{\prime}, t\right) f_{j}\left(\mathbf{r}_{j}^{\prime}, \boldsymbol{c}_{j}^{\prime}, t\right)-f_{i}\left(\mathbf{r}_{i}, \boldsymbol{c}_{i}^{\prime}, t\right) f_{j}\left(\mathbf{r}_{i}, \boldsymbol{c}_{j}^{\prime}, t\right)\right),
$$


we may accordingly split the operator $\mathcal{J}_{i}(f)$ into

$$
\mathcal{J}_{i}(f)=\mathcal{J}_{i}^{(0)}(f)+\mathcal{J}_{i}^{(1)}(f),
$$

where

$$
\begin{gathered}
\mathcal{J}_{i}^{(0)}(f)=\sum_{j \in \mathfrak{S}} \int \theta_{i j} f_{i}\left(\mathbf{r}_{i}, \boldsymbol{c}_{i}^{\prime}, t\right) f_{j}\left(\mathbf{r}_{i}, \boldsymbol{c}_{j}^{\prime}, t\right) \mathrm{d} \boldsymbol{x}_{j}, \\
\mathcal{J}_{i}^{(1)}(f)=\sum_{j \in \mathfrak{S}} \int \theta_{i j}\left(f_{i}\left(\mathbf{r}_{i}^{\prime}, \boldsymbol{c}_{i}^{\prime}, t\right) f_{j}\left(\mathbf{r}_{i}^{\prime}, \boldsymbol{c}_{j}^{\prime}, t\right)-f_{i}\left(\mathbf{r}_{i}, \boldsymbol{c}_{i}^{\prime}, t\right) f_{j}\left(\mathbf{r}_{i}, \boldsymbol{c}_{j}^{\prime}, t\right)\right) \mathrm{d} \boldsymbol{x}_{j} .
\end{gathered}
$$

The generalized Boltzmann equations may finally be written in the form

$$
\partial_{t} f_{i}+\boldsymbol{c}_{i} \cdot \nabla_{\mathbf{r}_{i}} f_{i}=\mathcal{J}_{i}^{(0)}(f)+\mathcal{J}_{i}^{(1)}(f),
$$

and Bogoliubov has established that the operators $\mathcal{J}_{i}^{0}(f)$ coincide with Boltzmann operators $[66,57]$. One may also note that the operator $\mathcal{J}_{i}^{(0)}$ only involves the particular distributions $f_{i}\left(\mathbf{r}_{i}, \boldsymbol{c}_{i}^{\prime}, t\right) f_{j}\left(\mathbf{r}_{i}, \boldsymbol{c}_{j}^{\prime}, t\right)$ whereas the terms $\mathcal{J}_{i}^{(1)}(f)$ may be interpreted as nonlocal perturbations arising from the difference of spatial positions during collisions.

\subsection{Differential expressions for $\mathcal{J}_{i}^{(1)}$}

We rewrite in this section the perturbed collision operators $\mathcal{J}_{i}^{(1)}, i \in \mathfrak{S}$, as sums of integro-differential operators for future use. To this aim, we first describe the traditional splitting of $\mathbf{r}_{i}^{\prime}$ and $\mathbf{r}_{j}^{\prime}$ using the invariance of centers of mass during collisions $[57,50]$. The center of mass $\mathbf{r}_{i j}^{\mathrm{m}}$ during a collision between particles of the $i$ th and $j$ th species may be written

$$
\mathbf{r}_{i j}^{\mathrm{m}}=\mu_{i} \mathbf{r}_{i}+\mu_{j} \mathbf{r}_{j}
$$

where $\mu_{i}$ and $\mu_{j}$ are the mass ratios for the $(i, j)$ th pair

$$
\mu_{i}=\frac{m_{i}}{m_{i}+m_{j}}, \quad \mu_{j}=\frac{m_{j}}{m_{i}+m_{j}} .
$$

From the invariance of $\mathbf{r}_{i j}^{\mathrm{m}}$ in a collision we have $\tau_{i j} \mathbf{r}_{i j}^{\mathrm{m}}=\mathbf{r}_{i j}^{\mathrm{m}}$ and from $\mathbf{r}_{i j}=\mathbf{r}_{j}-\mathbf{r}_{i}$ we also have $\mathbf{r}_{i j}^{\mathrm{m}}=\mathbf{r}_{i}+\mu_{j} \mathbf{r}_{i j}$. After some algebra, it is obtained that

$$
\begin{aligned}
& \mathbf{r}_{i}^{\prime}=\mathbf{r}_{i}+\mu_{j} \mathbf{r}_{i j}-\mu_{j} \mathbf{r}_{i j}^{\prime} \\
& \mathbf{r}_{j}^{\prime}=\mathbf{r}_{i}+\mu_{j} \mathbf{r}_{i j}+\mu_{i} \mathbf{r}_{i j}^{\prime},
\end{aligned}
$$

where $\mathbf{r}_{i j}^{\prime}=\mathbf{r}_{j}^{\prime}-\mathbf{r}_{i}^{\prime}$. In order to split $\mathcal{J}_{i}^{(1)}, i \in \mathfrak{S}$, we then write that

$$
\begin{gathered}
\delta \mathbf{r}_{i}=\mathbf{r}_{i}^{\prime}-\mathbf{r}_{i}=\mathbf{r}_{i}^{\prime}-\mathbf{r}_{i j}^{\mathrm{m}}+\mathbf{r}_{i j}^{\mathrm{m}}-\mathbf{r}_{i}, \\
\delta \mathbf{r}_{j}=\mathbf{r}_{j}^{\prime}-\mathbf{r}_{i}=\mathbf{r}_{j}^{\prime}-\mathbf{r}_{i j}^{\mathrm{m}}+\mathbf{r}_{i j}^{\mathrm{m}}-\mathbf{r}_{i},
\end{gathered}
$$

and use that $\mathbf{r}_{i j}^{\mathrm{m}}-\mathbf{r}_{i}=\mu_{j} \mathbf{r}_{i j}$ whereas $\mathbf{r}_{i}^{\prime}-\mathbf{r}_{i j}^{\mathrm{m}}=-\mu_{j} \mathbf{r}_{i j}^{\prime}$ and $\mathbf{r}_{j}^{\prime}-\mathbf{r}_{i j}^{\mathrm{m}}=\mu_{i} \mathbf{r}_{i j}^{\prime}$.

From the expression (3.34) of $\mathcal{J}_{i}^{(1)}(f)$ as well as (3.39) and (3.40), we obtain

$$
\mathcal{J}_{i}^{(1)}(f)=\mathcal{J}_{i}^{(1), s}(f)+\mathcal{J}_{i}^{(1), a}(f),
$$

where

$$
\mathcal{J}_{i}^{(1), a}(f)=\sum_{j \in \mathfrak{S}} \int \theta_{i j}\left(f_{i}\left(\mathbf{r}_{i}^{\prime}, \boldsymbol{c}_{i}^{\prime}, t\right) f_{j}\left(\mathbf{r}_{j}^{\prime}, \boldsymbol{c}_{j}^{\prime}, t\right)-f_{i}\left(\mathbf{r}_{i j}^{\mathrm{m}}, \boldsymbol{c}_{i}^{\prime}, t\right) f_{j}\left(\mathbf{r}_{i j}^{\mathrm{m}}, \boldsymbol{c}_{j}^{\prime}, t\right)\right) \mathrm{d} \boldsymbol{x}_{j},
$$




$$
\mathcal{J}_{i}^{(1), s}(f)=\sum_{j \in \mathfrak{S}} \int \theta_{i j}\left(f_{i}\left(\mathbf{r}_{i j}^{\mathrm{m}}, \boldsymbol{c}_{i}^{\prime}, t\right) f_{j}\left(\mathbf{r}_{i j}^{\mathrm{m}}, \boldsymbol{c}_{j}^{\prime}, t\right)-f_{i}\left(\mathbf{r}_{i}, \boldsymbol{c}_{i}^{\prime}, t\right) f_{j}\left(\mathbf{r}_{i}, \boldsymbol{c}_{j}^{\prime}, t\right)\right) \mathrm{d} \boldsymbol{x}_{j} .
$$

The operators $\mathcal{J}_{i}^{(1), a}$ measure the collision effects with respect to the centers of mass whereas the operators $\mathcal{J}_{i}^{(1), s}$ measure the shift between the local coordinates and the centers of mass.

Letting for convenience

$$
f_{i j}^{\mathrm{cl}}\left(\mathbf{r}, \boldsymbol{c}_{i}, \boldsymbol{c}_{j}, t\right)=f_{i}\left(\mathbf{r}, \boldsymbol{c}_{i}^{\prime}, t\right) f_{j}\left(\mathbf{r}, \boldsymbol{c}_{j}^{\prime}, t\right),
$$

and using that $\mathbf{r}_{i j}^{\mathrm{m}}-\mathbf{r}_{i}=\mu_{j} \mathbf{r}_{i j}$, we obtain after some calculus that

$$
\mathcal{J}_{i}^{(1), s}(f)=\sum_{j \in \mathfrak{S}} \mu_{j} \int \theta_{i j} \partial_{\mathbf{r}} f_{i j}^{\mathrm{cl}}\left(\alpha \mathbf{r}_{i j}^{\mathrm{m}}+(1-\alpha) \mathbf{r}_{i}, \boldsymbol{c}_{i}, \boldsymbol{c}_{j}, t\right) \cdot \mathbf{r}_{i j} \mathrm{~d} \alpha \mathrm{d} \boldsymbol{x}_{j}
$$

Similarly, since $\mathbf{r}_{i}^{\prime}-\mathbf{r}_{i j}^{\mathrm{m}}=-\mu_{j} \mathbf{r}_{i j}^{\prime}$ and $\mathbf{r}_{j}^{\prime}-\mathbf{r}_{i j}^{\mathrm{m}}=\mu_{i} \mathbf{r}_{i j}^{\prime}$, we have

$$
\mathcal{J}_{i}^{(1), a}(f)=-\sum_{j \in \mathfrak{S}} \mu_{i j} \int \theta_{i j} \Xi_{i j}\left(\alpha, \mathbf{r}_{i j}^{\mathrm{m}}, \mathbf{r}_{i j}^{\prime}, \boldsymbol{c}_{i}^{\prime}, \boldsymbol{c}_{j}^{\prime}, t\right) \cdot \mathbf{r}_{i j}^{\prime} \mathrm{d} \alpha \mathrm{d} \boldsymbol{x}_{j}
$$

where

$$
\begin{aligned}
\Xi_{i j}\left(\alpha, \mathbf{r}_{i j}^{\mathrm{m}}, \mathbf{r}_{i j}^{\prime}, \boldsymbol{c}_{i}^{\prime}, \boldsymbol{c}_{j}^{\prime}, t\right)= & \frac{1}{m_{i}} \partial_{\mathbf{r}} f_{i}\left(\mathbf{r}_{i j}^{\mathrm{m}}-\alpha \mu_{j} \mathbf{r}_{i j}^{\prime}, \boldsymbol{c}_{i}^{\prime}, t\right) f_{j}\left(\mathbf{r}_{i j}^{\mathrm{m}}+\alpha \mu_{i} \mathbf{r}_{i j}^{\prime}, \boldsymbol{c}_{j}^{\prime}, t\right) \\
& -f_{i}\left(\mathbf{r}_{i j}^{\mathrm{m}}-\alpha \mu_{j} \mathbf{r}_{i j}^{\prime}, \boldsymbol{c}_{i}^{\prime}, t\right) \frac{1}{m_{j}} \partial_{\mathbf{r}} f_{j}\left(\mathbf{r}_{i j}^{\mathrm{m}}+\alpha \mu_{i} \mathbf{r}_{i j}^{\prime}, \boldsymbol{c}_{j}^{\prime}, t\right)
\end{aligned}
$$

The perturbed collision operator $\mathcal{J}_{i}^{(1)}(f)$ may thus be written as the sum of two integrodifferential operators $\mathcal{J}_{i}^{(1), a}$ and $\mathcal{J}_{i}^{(1), s}$.

The transformed coordinates $\mathbf{r}_{i}^{\prime}$ and $\mathbf{r}_{j}^{\prime}$, and the transformed velocities $\boldsymbol{c}_{i}^{\prime}$ and $\boldsymbol{c}_{j}^{\prime}$, are often used in kinetic developments concerning dense gases because they cannot be expressed simply in terms of $\mathbf{r}_{i}, \mathbf{r}_{j}, \boldsymbol{c}_{i}$ and $\boldsymbol{c}_{j}$. Only the terms arising from the center of mass $\mathbf{r}_{i j}^{\mathrm{m}}$ and associated with $\mathcal{J}_{i}^{(1), s}$ appear to be convenient to manipulate.

\subsection{Kinetic entropy}

A kinetic entropy for dense gas mixtures has been introduced by Cohen et al. [50] and reads

$$
\begin{aligned}
\mathcal{S}^{\mathrm{K}}=-\sum_{i \in \mathfrak{S}} & k_{\mathrm{B}} \int f_{i}\left(\log \left(\mathrm{h}_{\mathrm{P}}^{3} f_{i} / m_{i}^{3}\right)-1\right) \mathrm{d} \boldsymbol{c}_{i} \\
& \quad-\frac{1}{2} \sum_{i, j \in \mathfrak{S}} k_{\mathrm{B}} \int\left(f_{i j}\left(\log \left(f_{i j} / f_{i} f_{j}\right)-1\right)+f_{i} f_{j}\right) \mathrm{d} \boldsymbol{c}_{i} \mathrm{~d} \mathbf{r}_{i j} \mathrm{~d} \boldsymbol{c}_{j},
\end{aligned}
$$

where $h_{P}$ is the Planck constant. In the situation of single species fluids, similar entropies have been introduced in a pioneering work by Stratonovich [73] and investigated by Nettleton and Green [74], and the entropy (3.47) has also been used by Klimontovich [75]

An important point about such kinetic entropies is that, contrarily to the case of dilute fluids, there is no known $\mathrm{H}$ theorem for spatially nonuniform systems governed by the generalized Boltzmann equations (3.24). An H-theorem has only been established for 
single species fluids in the special situation of spatially homogeneous fluids by Klimontovich [75]. The entropies of systems of particles for the BBGKY hierarchy have also been investigated by Martynov who established that there are entropy exchanges between the different levels of approximations [76] and by Singer who discussed different truncations of Kirkwood type for the BBGKY hierarchy [77]

\section{Nonideal multicomponent fluids with normal diffusion}

We investigate in this section the equation governing nonideal multicomponent fluids with normal diffusive processes, i.e., without diffuse interface terms. To this aim, we introduce a proper scaling for the generalized Boltzmann equations (3.35) and use the ChapmanEnskog method. This new scaling clarifies the apparition of nonzero contributions from the collision terms $\mathcal{J}_{i}$ in the fluid equations. It also directly yields that equilibrium distributions are Maxwellian distributions at zeroth order and will allow the derivation of Cahn-Hilliard fluid equations. This derivation of nonideal fluid governing equations only uses first order Taylor expansions of pair distribution functions in the fluxes. The resulting nonideal thermodynamic properties will be denoted with the superscript ${ }^{\mathrm{u}}$ to be consistent with Section 2. We also simplify some of the collision integrals for dense gas mixtures, typically assuming that repulsive potentials between pair of particles are hard. As a result, the diffusive fluxes are directly expressed in terms of chemical potential gradients, as expected from thermodynamics, without linear system inversions as in previous work $[50,51]$. We also avoid the use of pressure based thermodynamic functions-like the species enthalpies or entropies - for expressing the diffusion fluxes [50, 51], since such thermodynamic functions may explode at unstable thermodynamic states [9].

\subsection{A new Enskog scaling}

The generalized Boltzmann equations (3.35) are written in the form

$$
\partial_{t} f_{i}+\boldsymbol{c}_{i} \cdot \nabla_{\mathbf{r}_{i}} f_{i}=\frac{1}{\epsilon} \mathcal{J}_{i}^{(0)}(f)+\mathcal{J}_{i}^{(1)}(f)
$$

where $\epsilon$ is the formal parameter associated with Enskog expansion. The distributions $f_{i}$, $i \in \mathfrak{S}$, are correspondingly expanded in the form

$$
f_{i}=f_{i}^{(0)}\left(1+\epsilon \phi_{i}^{(1)}+\mathcal{O}\left(\epsilon^{2}\right)\right),
$$

where $\phi_{i}^{(1)}$ is the perturbed distribution for the $i$ th species in the Navier-Stokes regime.

The traditional scaling of the collision operators $\mathcal{J}_{i}$ used in previous work has been in the form $\mathcal{J}_{i} / \epsilon$ instead of $\mathcal{J}_{i}^{(0)} / \epsilon$. This leads to conceptual difficulties since there are invariably nonzero gradient terms arising from $\mathcal{J}_{i}$ in the Euler and Navier-Stokes equations $[52,56,57,58,54]$. Since these terms arise from $\mathcal{J}_{i}^{(1)}$, the new scaling leads to a clearer derivation. Moreover, with the traditional scaling, it is necessary to assume at zeroth order that the distributions are Maxwellians [56, 57, 50], whereas it will be simply derived by using (4.1). Finally, we do not use a distinguished limit where the Enskog parameter is taken to be equal to a typical rescaled macroscopic gradient $[52,56,57,58,54]$. This will notably allows the derivation of Cahn-Hilliard type equations where typically first order derivatives are mixed with third order derivatives. 


\subsection{The Euler regime}

We investigate in this section the zeroth order or Euler regime and the corresponding kinetic equations are

$$
\mathcal{J}_{i}^{(0)}\left(f^{(0)}\right)=0, \quad i \in \mathfrak{S},
$$

where $f^{(0)}=\left(f_{i}^{(0)}\right)_{i \in \mathfrak{S}}$. Keeping in mind that the operators $\mathcal{J}_{i}^{(0)}$ coincide with the Boltzmann collision operators [66, 57], and using Boltzmann $\mathrm{H}$ theorem, we obtain that the distributions $f_{i}^{(0)}$ are local Maxwellians

$$
f_{i}^{(0)}=n_{i}\left(\frac{m_{i}}{2 \pi k_{\mathrm{B}} T}\right)^{\frac{3}{2}} \exp \left(-\frac{m_{i}\left|\boldsymbol{c}_{i}-\boldsymbol{v}\right|^{2}}{2 k_{\mathrm{B}} T}\right), \quad i \in \mathfrak{S},
$$

where $n_{i}$ denotes the number density of the $i$ th species, $m_{i}$ the mass of the particles of the $i$ th species, $\boldsymbol{v}$ the local fluid velocity and $T$ the local fluid temperature. The temperature $T$ insures that the local value of the internal energy density $\mathcal{E}$ is recovered with $f^{(0)}=\left(f_{i}^{(0)}\right)_{i \in \mathfrak{S}}$, including its potential part [50, 51, 54, 57].

The zeroth order pair distributions $f_{i j}^{(0)}$ are then evaluated from (3.44) and are in the form $[57,54,55,50]$

$$
f_{i j}^{(0), \mathrm{cl}}=f_{i}^{(0)}\left(\mathbf{r}_{i}, \boldsymbol{c}_{i}^{\prime}, t\right) f_{j}^{(0)}\left(\mathbf{r}_{i}, \boldsymbol{c}_{j}^{\prime}, t\right),
$$

with both distributions $f_{i}^{(0)}$ and $f_{j}^{(0)}$ evaluated at $\mathbf{r}=\mathbf{r}_{i}=\mathbf{r}_{j}$ as suggested by the kernel of $\mathcal{J}^{(0)}=\left(\mathcal{J}_{i}^{(0)}\right)_{i \in \mathfrak{S}}[52,57,54,50]$. From the conservation of momentum (3.28) and energy (3.29) these zeroth order distribution $f_{i j}^{(0), c l}$ may be rewritten as

$$
f_{i j}^{(0), \mathrm{cl}}=f_{i}^{(0)}\left(\mathbf{r}_{i}, \boldsymbol{c}_{i}, t\right) f_{j}^{(0)}\left(\mathbf{r}_{i}, \boldsymbol{c}_{j}, t\right) g_{i j}\left(\mathbf{r}_{i}, \mathbf{r}_{i j}\right),
$$

where $g_{i j}\left(\mathbf{r}_{i}, \mathbf{r}_{i j}\right)$ denotes the equilibrium correlation function

$$
g_{i j}\left(\mathbf{r}_{i}, r_{i j}\right)=\exp \left(-\frac{\varphi_{i j}\left(r_{i j}\right)}{k_{\mathrm{B}} T\left(\mathbf{r}_{i}\right)}\right)
$$

evaluated at temperature $T\left(\mathbf{r}_{i}\right)$.

The nonideal multicomponent Euler fluid equations are then obtained at zeroth order $[52,56,57,58,54,50]$. In order to derive these equations, the most convenient method is to use the Maxwellian distributions (4.4), the pair correlation functions (4.5), and the macroscopic equations of Section 3.2 with first order Taylor expansions of pair distribution functions. This method is equivalent to directly take moments of the kinetic equations and use the differential expressions of the perturbed collision operators in order to evaluate $\mathcal{J}_{i}^{(1)}\left(f^{(0)}\right)$. The multicomponent Euler equations are obtained in the form [50]

$$
\begin{aligned}
& \partial_{t} \rho_{i}+\nabla \cdot\left(\rho_{i} \boldsymbol{v}\right)=0, \quad i \in \mathfrak{S}, \\
& \partial_{t}(\rho \boldsymbol{v})+\boldsymbol{\nabla} \cdot(\rho \boldsymbol{v} \otimes \boldsymbol{v})+\nabla p^{\mathrm{u}}=0, \\
& \partial_{t} \mathcal{E}^{\mathrm{u}}+\boldsymbol{\nabla} \cdot\left(\boldsymbol{v} \mathcal{E}^{\mathrm{u}}\right)+p^{\mathrm{u}} \boldsymbol{\nabla} \cdot \boldsymbol{v}=0,
\end{aligned}
$$

where the internal energy $\mathcal{E}^{\mathrm{u}}$ and the pressure $p^{\mathrm{u}}$ are detailed in the next section. The zeroth order pressure tensor thus reduces to $\mathcal{P}^{(0)}=p^{\mathrm{u}} \boldsymbol{I}$ and the zeroth order mass and heat diffusion fluxes vanish $\overline{\mathcal{F}}_{i}^{(0)}=0, i \in \mathfrak{S}$, and $\mathcal{Q}^{(0)}=0$. 


\subsection{Thermodynamic properties}

Using the one and two-particle distribution functions (4.4) and (4.5), and the macroscopic equations of Section (3.2), the pressure is found in the form

$$
p^{\mathrm{u}}=\sum_{i \in \mathfrak{S}} n_{i} k_{\mathrm{B}} T-\sum_{i, j \in \mathfrak{S}} \frac{1}{6} n_{i} n_{j} \int \varphi_{i j}^{\prime}\left(r_{i j}\right) r_{i j} g_{i j}\left(r_{i j}\right) \mathrm{d} \mathbf{r}_{i j}
$$

Integrating by parts the integrals in (4.10), it is obtained that

$$
\begin{aligned}
\frac{1}{6} \int \varphi_{i j}^{\prime}\left(r_{i j}\right) r_{i j} g_{i j}\left(\mathbf{r}_{i}, r_{i j}\right) \mathrm{d} \mathbf{r}_{i j} & =\frac{2 \pi}{3} \int \varphi_{i j}^{\prime}\left(r_{i j}\right) r_{i j}^{3} g_{i j}\left(\mathbf{r}_{i}, r_{i j}\right) \mathrm{d} r_{i j} \\
& =2 \pi k_{\mathrm{B}} T \int \mathfrak{f}_{i j} r_{i j}^{2} \mathrm{~d} r_{i j}=\frac{1}{2} k_{\mathrm{B}} T \int \mathfrak{f}_{i j} \mathrm{~d} \mathbf{r}_{i j},
\end{aligned}
$$

where $\mathfrak{f}_{i j}$ denote the $(i, j)$ th species pair Mayer function

$$
\mathfrak{f}_{i j}\left(\mathbf{r}_{i}, \mathbf{r}_{i j}\right)=\exp \left(-\frac{\varphi_{i j}\left(r_{i j}\right)}{k_{\mathrm{B}} T\left(\mathbf{r}_{i}\right)}\right)-1 .
$$

Denoting by $\beta_{i j}$ the $(i, j)$ th species pair second virial coefficients

$$
\beta_{i j}=\int \mathfrak{f}_{i j} \mathrm{~d} \mathbf{r}_{i j},
$$

the pressure is then in the form [50]

$$
p^{\mathrm{u}}=\sum_{i \in \mathfrak{S}} n_{i} k_{\mathrm{B}} T-\sum_{i, j \in \mathfrak{S}} \frac{1}{2} n_{i} n_{j} \beta_{i j} k_{\mathrm{B}} T
$$

The internal energy is derived with a similar procedure and reads

$$
\mathcal{E}^{\mathrm{u}}=\frac{3}{2} \sum_{i \in \mathfrak{S}} n_{i} k_{\mathrm{B}} T+\frac{1}{2} \sum_{i, j \in \mathfrak{S}} n_{i} n_{j} \int \varphi_{i j}\left(r_{i j}\right) g_{i j}\left(r_{i j}\right) \mathrm{d} \mathbf{r}_{i j}
$$

so that [50]

$$
\mathcal{E}^{\mathrm{u}}=\frac{3}{2} \sum_{i \in \mathfrak{S}} n_{i} k_{\mathrm{B}} T+\frac{1}{2} \sum_{i, j \in \mathfrak{S}} n_{i} n_{j} \beta_{i j}^{\prime}\left(k_{\mathrm{B}} T\right)^{2},
$$

where $\beta_{i j}^{\prime}=\mathrm{d} \beta_{i j} / \mathrm{d}\left(k_{\mathrm{B}} T\right)$.

The fluid entropy is obtained by evaluating the kinetic entropy (3.47) with Maxwellian distributions (4.4) and the pair distribution functions (4.5). After some algebra, it is found that the volumetric entropy $\mathcal{S}^{\mathrm{u}}$ is in the form [50]

$$
\mathcal{S}^{\mathrm{u}}=-\sum_{i \in \mathfrak{S}} k_{\mathrm{B}} n_{i}\left(\log \left(n_{i} \Lambda_{i}^{3}\right)-\frac{5}{2}\right)+\sum_{i, j \in \mathfrak{S}} \frac{1}{2} k_{\mathrm{B}} n_{i} n_{j}\left(\beta_{i j}+k_{\mathrm{B}} T \beta_{i j}^{\prime}\right),
$$

where $\Lambda_{i}$ is the De Broglie thermal wavelength $\Lambda_{i}=\mathrm{h}_{\mathrm{P}}\left(2 \pi m_{i} k_{\mathrm{B}} T\right)^{-1 / 2}$ of the $i$ th species. This entropy has been shown to be second order accurate by Cohen et al. [50], that is, one may establish that formally $\int \mathcal{S}^{\mathrm{K}} \mathrm{d} \boldsymbol{c}_{i}-\mathcal{S}^{\mathrm{u}}=\mathcal{O}\left(\epsilon^{2}\right)$.

The corresponding species Gibbs functions per molecule are next obtained in the form

$$
g_{i}^{\mathrm{u}}=k_{\mathrm{B}} T \log \left(n_{i} \Lambda_{i}^{3}\right)-\sum_{j \in \mathfrak{S}} k_{\mathrm{B}} T n_{j} \beta_{i j},
$$


and play an important role in diffusive processes. The mixture Gibbs function $\mathcal{G}^{\mathrm{u}}$ is then given by $\mathcal{G}^{\mathrm{u}}=\sum_{k \in \mathfrak{S}} n_{k} g_{i}^{\mathrm{u}}$. Cohen et al. [50] have also established that the volumetric Gibbs relation $\mathrm{d} \mathcal{S}^{\mathrm{u}}=\mathrm{d} \mathcal{E}^{\mathrm{u}}-\sum_{k \in \mathfrak{S}} g_{k}^{\mathrm{u}} \mathrm{d} n_{k}$ holds at second order.

Another interesting property of the species Gibbs functions $g_{i}^{\mathrm{u}}, i \in \mathfrak{S}$, is that they are naturally pressure based, that is, the derivative of $g^{\mathrm{u}}=\mathcal{G}^{\mathrm{u}} / n$ with respect to the mole fraction of the $i$ th species $\mathrm{x}_{i}=n_{i} / n$ at fixed temperature, pressure, and other mole fractions coincides with $g_{i}^{\mathrm{u}}[78]$. These species Gibbs functions $g_{i}^{\mathrm{u}}, i \in \mathfrak{S}$, notably remain finite at states of mechanical instability when $\partial_{n} p^{\mathrm{u}}=0$. On the contrary, various traditional pressure-based thermodynamic functions like species enthalpies, entropies, or specific heats explode at unstable thermodynamic state where $\partial_{n} p^{\mathrm{u}}=0$, that is, when $\sum_{i, j \in \mathfrak{S}} n_{i} n_{j} \beta_{i j}=n[9]$. Such exploding thermodynamic functions have notably been used by Cohen et al. [50] and Braun et al. [51] in order to express the mass and heat fluxes, so that such expressions are only valid when thermodynamic stability holds.

\subsection{Linearized equations}

At first order, taking into account the operator decomposition (3.32), the linearized equations governing the perturbed distribution functions $\phi^{(1)}=\left(\phi_{i}^{(1)}\right)_{i \in \mathfrak{S}}$ are in the form

$$
\mathcal{I}_{i}\left(\phi^{(1)}\right)=\psi_{i}^{(1)}, \quad i \in \mathfrak{S},
$$

where the $i$ th linearized operator $\mathcal{I}_{i}$ reads

$$
\mathcal{I}_{i}\left(\phi^{(1)}\right)=-\frac{1}{f_{i}^{(0)}} \sum_{j \in \mathfrak{S}} \int \theta_{i j} f_{i}^{(0)}\left(\mathbf{r}_{i}, \boldsymbol{c}_{i}^{\prime}, t\right) f_{j}^{(0)}\left(\mathbf{r}_{i}, \boldsymbol{c}_{j}^{\prime}, t\right)\left(\phi_{i}^{(1)}\left(\mathbf{r}_{i}, \boldsymbol{c}_{i}^{\prime}, t\right)+\phi_{j}^{(1)}\left(\mathbf{r}_{i}, \boldsymbol{c}_{j}^{\prime}, t\right)\right) \mathrm{d} \boldsymbol{x}_{j},
$$

and the right hand sides $\psi_{i}$ are

$$
\psi_{i}^{(1)}=-\left(\partial_{t} \log f_{i}^{(0)}+\boldsymbol{c}_{i} \cdot \nabla_{\mathbf{r}_{i}} \log f_{i}^{(0)}\right)+\frac{1}{f_{i}^{(0)}} \mathcal{J}_{i}^{(1)}\left(f^{(0)}\right), \quad i \in \mathfrak{S} .
$$

A fundamental property is now that the linearized operators $\mathcal{I}_{i}$ coincide with Boltzmann linearized collision operators $[66,57]$.

The right hand sides $\psi^{(1)}=\left(\psi_{i}^{(1)}\right)_{i \in \mathfrak{S}}$ are evaluated in Appendix D and may be written

$$
\psi_{i}^{(1)}=-\psi_{i}^{\eta}: \nabla \boldsymbol{v}-\psi_{i}^{\kappa} \nabla \cdot \boldsymbol{v}-\sum_{j \in \mathfrak{S}} \psi_{i}^{D_{j}} \cdot \boldsymbol{X}_{j}^{\mathrm{th}}-\psi_{i}^{\widehat{\lambda}} \cdot \boldsymbol{\nabla}\left(\frac{-1}{k_{\mathrm{B}} T}\right),
$$

where $\psi_{i}^{\eta}$ are symmetric traceless second order tensors, $\psi_{i}^{\kappa}$ are scalars, and $\psi^{D_{j}}, j \in \mathfrak{S}$, $\psi_{i}^{\widehat{\lambda}}$, are vectors. The quantity $\boldsymbol{X}_{i}^{\text {th }}$ denotes the normal force term of the $i$ th species arising solely from thermodynamics, i.e., without capillary effects

$$
\boldsymbol{X}_{i}^{\mathrm{th}}=n_{i} k_{\mathrm{B}} T \nabla\left(\frac{g_{i}^{\mathrm{u}}}{k_{\mathrm{B}} T}\right)=\rho_{i} T \nabla\left(\frac{\bar{g}_{i}^{\mathrm{u}}}{T}\right),
$$

where $g_{i}^{\mathrm{u}}$ is the Gibbs function of the $i$ th species per particle and $\bar{g}_{i}^{\mathrm{u}}=g_{i}^{\mathrm{u}} / m_{i}$ the Gibbs function per unit mass. This force term $\boldsymbol{X}_{i}^{\text {th }}$ is the thermodynamic part of the total force term $\boldsymbol{X}_{i}$ obtained in (2.22). This force term $\boldsymbol{X}_{i}^{\text {th }}$ is also directly related to the thermodynamic diffusion driving force $\boldsymbol{X}_{i}^{\text {th }}=n k_{\mathrm{B}} T \boldsymbol{d}_{i}^{\text {th }}, i \in \mathfrak{S}$. As detailed in Appendix D, the 
functions $\psi_{i}^{\eta}, \psi_{i}^{\kappa}, \psi_{i}^{D_{j}}, j \in \mathfrak{S}$, and $\psi_{i}^{\widehat{\lambda}}$, are given by

$$
\begin{aligned}
& \psi_{i}^{\eta}=\frac{m_{i}}{k_{\mathrm{B}} T}\left(\left(\boldsymbol{c}_{i}-\boldsymbol{v}\right) \otimes\left(\boldsymbol{c}_{i}-\boldsymbol{v}\right)-\frac{1}{3}\left|\boldsymbol{c}_{i}-\boldsymbol{v}\right|^{2} \boldsymbol{I}\right)\left(1-\sum_{j \in \mathfrak{S}} \frac{n_{j} m_{j} \beta_{i j}}{m_{i}+m_{j}}\right)+\sum_{j \in \mathfrak{S}} \mu_{i j} n_{j} \overline{\mathfrak{a}}_{i j}^{\eta}, \\
& \psi_{i}^{\kappa}=-\left(\frac{3}{2}-\frac{m_{i}\left|\boldsymbol{c}_{i}-\boldsymbol{v}\right|^{2}}{2 k_{\mathrm{B}} T}\right)\left(\frac{2}{3}\left(1-\sum_{j \in \mathfrak{S}} \frac{n_{j} m_{j} \beta_{i j}}{m_{i}+m_{j}}\right)\right. \\
& \left.\quad+\frac{1}{T \partial_{T} \mathcal{E}^{\mathrm{u}}}\left(\sum_{l \in \mathfrak{S}} n_{l} \partial_{n_{l}} \mathcal{E}^{\mathrm{u}}-\mathcal{E}^{\mathrm{u}}-p^{\mathrm{u}}\right)\right)+\sum_{j \in \mathfrak{S}} \mu_{i j} n_{j} \overline{\mathfrak{a}}_{i j}^{\kappa}, \\
& \psi_{i}^{D_{j}}=\frac{1}{n_{i} k_{\mathrm{B}} T}\left(\delta_{i j}-\mathrm{y}_{i}\right)\left(\boldsymbol{c}_{i}-\boldsymbol{v}\right), \\
& \psi_{i}^{\widehat{\lambda}}=-\left(\boldsymbol{c}_{i}-\boldsymbol{v}\right)\left(\frac{3}{2} k_{\mathrm{B}} T-\frac{m_{i}\left|\boldsymbol{c}_{i}-\boldsymbol{v}\right|^{2}}{2}\right)\left(1-\sum_{j \in \mathfrak{S}} \frac{n_{j} m_{j} \beta_{i j}}{m_{i}+m_{j}}\right) \\
& \quad+\left(\boldsymbol{c}_{i}-\boldsymbol{v}\right)\left(\frac{3}{2} k_{\mathrm{B}} T+\sum_{j \in \mathfrak{S}} \frac{n_{j} m_{i}}{m_{i}+m_{j}} \beta_{i j}^{\prime}\left(k_{\mathrm{B}} T\right)^{2}-\frac{m_{i}}{\rho}\left(\mathcal{E}^{\mathrm{u}}+p^{\mathrm{u}}\right)\right)+\sum_{j \in \mathfrak{S}} \mu_{i j} n_{j} \overline{\mathfrak{a}}_{i j}^{\widehat{\lambda}},
\end{aligned}
$$

where $\mu_{i j}=m_{i} m_{j} /\left(m_{i}+m_{j}\right)$ denotes the reduced mass of the $(i, j)$ th pair of particles, $\mathrm{y}_{i}=\rho_{i} / \rho$ the mass fraction of the $i$ th species and $\overline{\mathfrak{a}}_{i j}^{\eta}, \overline{\mathfrak{a}}_{i j}^{\kappa}$ and $\overline{\mathfrak{a}}_{i j}^{\widehat{\lambda}}$ are collision integrals associated with dense gas mixtures. Some collision integrals associated with dense gases $\overline{\mathfrak{a}}_{i j}^{D}, i, j \in \mathfrak{S}$, have been simplified by assuming that interaction potentials between pairs of particles are hard (D.11) as detailed in Appendix D. The first order equations (4.18)(4.21) are essentially similar to classical first order relations obtained for dilute gases, except that there are extra gradient terms arising from the perturbed collision operators $\mathcal{J}_{i}^{(1)}$ and that the force terms $\boldsymbol{X}_{i}^{\text {th }}, i \in \mathfrak{S}$, involve chemical potential gradients.

The distributions $\phi_{i}^{(1)}$ are then uniquely determined by imposing Enskog type constraints so that the true value of the local macroscopic variables $n_{i}, \boldsymbol{v}$ and $T$, or equivalently $\mathcal{E}$, are determined by the distributions $f_{i}^{(0)}$ alone [57, 54]. These constraints, summarized in Appendix E, have been shown to reduce to $[54,79,50]$

$$
\begin{array}{r}
\int f_{i}^{(0)} \phi_{i}^{(1)} \mathrm{d} \boldsymbol{c}_{i}=0, \\
\sum_{i \in \mathfrak{S}} \int m_{i}\left(\boldsymbol{c}_{i}-\boldsymbol{v}\right) f_{i}^{(0)} \phi_{i}^{(1)} \mathrm{d} \boldsymbol{c}_{i}=0, \\
\sum_{i \in \mathfrak{S}} \int \frac{1}{2} m_{i}\left|\boldsymbol{c}_{i}-\boldsymbol{v}\right|^{2} f_{i}^{(0)} \phi_{i}^{(1)} \mathrm{d} \boldsymbol{c}_{i}=0 .
\end{array}
$$

In particular, the potential and gradient parts are eliminated in the constraints [50]. By linearity and isotropy of the collision operator, we may decompose $\phi_{i}^{(1)}$ in the form

$$
\phi_{i}=-\phi_{i}^{\eta}: \nabla \boldsymbol{v}-\phi_{i}^{\kappa} \boldsymbol{\nabla} \cdot \boldsymbol{v}-\sum_{j \in \mathfrak{S}} \phi_{i}^{D_{j}} \cdot \boldsymbol{X}_{j}^{\mathrm{th}}-\phi_{i}^{\hat{\lambda}} \cdot \boldsymbol{\nabla}\left(\frac{-1}{k_{\mathrm{B}} T}\right),
$$

where $\phi_{i}^{\eta}$ are traceless second order symmetric tensor, $\phi_{i}^{\kappa}$ are scalars, $\phi^{D_{j}}, j \in \mathfrak{S}$, and $\phi_{i}^{\widehat{\lambda}}$, are vectors. 
Each unknown $\phi^{\mu}=\left(\phi_{i}^{\mu}\right)_{i \in \mathfrak{S}}$ for $\mu \in\left\{\eta, \kappa, D_{j}, \widehat{\lambda}\right\}$ then satisfies a system of tensorial integral equations $\mathcal{I}_{i}\left(\phi^{\mu}\right)=\psi_{i}^{\mu}, i \in \mathfrak{S}$, and the $\phi^{\mu}=\left(\phi_{i}^{\mu}\right)_{i \in \mathfrak{S}}$ have the following structure [50]

$$
\begin{aligned}
\phi_{i}^{\eta} & =\varphi_{i}^{\eta}\left(\left|\boldsymbol{c}_{i}-\boldsymbol{v}\right|^{2}\right)\left(\left(\boldsymbol{c}_{i}-\boldsymbol{v}\right) \otimes\left(\boldsymbol{c}_{i}-\boldsymbol{v}\right)-\frac{1}{3}\left|\boldsymbol{c}_{i}-\boldsymbol{v}\right|^{2} \boldsymbol{I}\right), \\
\phi_{i}^{\kappa} & =\varphi_{i}^{\kappa}\left(\left|\boldsymbol{c}_{i}-\boldsymbol{v}\right|^{2}\right), \\
\phi_{i}^{D_{j}} & =\varphi_{i}^{D_{j}}\left(\left|\boldsymbol{c}_{i}-\boldsymbol{v}\right|^{2}\right)\left(\boldsymbol{c}_{i}-\boldsymbol{v}\right), \quad j \in \mathfrak{S}, \\
\phi_{i}^{\widehat{\lambda}} & =\varphi_{i}^{\widehat{\lambda}}\left(\left|\boldsymbol{c}_{i}-\boldsymbol{v}\right|^{2}\right)\left(\boldsymbol{c}_{i}-\boldsymbol{v}\right),
\end{aligned}
$$

where $\varphi_{i}^{\eta}, \varphi_{i}^{\kappa}, \varphi_{i}^{D_{j}}$ and $\varphi_{i}^{\widehat{\lambda}}$ are scalar isotropic functions of the velocities, i.e., that only depend on $\left|\boldsymbol{c}_{i}-\boldsymbol{v}\right|^{2}$. The tensorial distributions $\phi^{\mu}=\left(\phi_{i}^{\mu}\right)_{i \in \mathfrak{S}}$ further satisfy the induced constraints derived from (4.29)-(4.31) according to their tensorial rank

$$
\begin{aligned}
& \int f_{i}^{(0)} \phi_{i}^{\kappa} \mathrm{d} \boldsymbol{c}_{i}=0, \quad i \in \mathfrak{S}, \quad \sum_{i \in \mathfrak{S}} \int \frac{1}{2} m_{i}\left|\boldsymbol{c}_{i}-\boldsymbol{v}\right|^{2} f_{i}^{(0)} \phi_{i}^{\kappa} \mathrm{d} \boldsymbol{c}_{i}=0, \\
& \sum_{i \in \mathfrak{S}} \int m_{i}\left(\boldsymbol{c}_{i}-\boldsymbol{v}\right) f_{i}^{(0)} \phi_{i}^{D_{j}} \mathrm{~d} \boldsymbol{c}_{i}=0, \quad j \in \mathfrak{S}, \\
& \sum_{i \in \mathfrak{S}} \int m_{i}\left(\boldsymbol{c}_{i}-\boldsymbol{v}\right) f_{i}^{(0)} \phi_{i}^{\hat{\lambda}} \mathrm{d} \boldsymbol{c}_{i}=0 .
\end{aligned}
$$

\subsection{The Navier-Stokes-Fourier-Fick regime}

\subsubsection{Dissipative fluxes for all thermodynamic states}

The resulting macroscopic equations at first order of Enskog expansion are of NavierStokes-Fourier-Fick type [50]. The situation of single species fluids, that only involves the fluid and energy equations, has been discussed in $[52,56,57,58,54]$.

By substituting the expansion (4.32) into the expression of the diffusion fluxes (3.11), pressure tensors (3.14)(3.15), and heat fluxes (3.19)(3.20), it is obtained after some algebra that

$$
\mathcal{P}=p^{\mathrm{u}} \boldsymbol{I}+\mathcal{P}^{\mathrm{d}}, \quad \overline{\mathcal{F}}_{i}=\overline{\mathcal{F}}_{i}^{\mathrm{d}}, \quad \mathcal{Q}=\mathcal{Q}^{\mathrm{d}},
$$

where

$$
\begin{aligned}
\mathcal{P}^{\mathrm{d}} & =-\mathfrak{v} \boldsymbol{\nabla} \cdot \boldsymbol{v} \boldsymbol{I}-\eta\left(\boldsymbol{\nabla} \boldsymbol{v}+\boldsymbol{\nabla} \boldsymbol{v}^{t}-\frac{2}{3} \boldsymbol{\nabla} \cdot \boldsymbol{v} \boldsymbol{I}\right), \\
\overline{\mathcal{F}}_{i}^{\mathrm{d}} & =-\sum_{j \in \mathfrak{S}} L_{i j} \boldsymbol{\nabla}\left(\frac{\bar{g}_{j}^{\mathrm{u}}}{T}\right)-L_{i \mathrm{e}} \boldsymbol{\nabla}\left(\frac{-1}{T}\right), \\
\mathcal{Q}^{\mathrm{d}} & =-\sum_{i \in \mathfrak{S}} L_{\mathrm{e} i} \boldsymbol{\nabla}\left(\frac{\bar{g}_{i}^{\mathrm{u}}}{T}\right)-L_{\mathrm{ee}} \boldsymbol{\nabla}\left(\frac{-1}{T}\right),
\end{aligned}
$$

and $\mathfrak{v}$ denotes the volume viscosity, $\eta$ the shear viscosity, $L_{i j}, i, j \in \mathfrak{S} \cup\{e\}$ the mass and heat transport coefficients, and $\bar{g}_{i}^{\mathrm{u}}$ the Gibbs function per unit mass of the $i$ th species. This thermodynamic form (4.38) and (4.39) for the diffusion and heat fluxes is then fully compatible with rational thermodynamics [59, 61].

Focusing in more details on the species mass fluxes $\overline{\mathcal{F}}_{i}=\int m_{i}\left(\boldsymbol{c}_{i}-\boldsymbol{v}\right) f_{i}^{(0)} \phi_{i}^{(1)} \mathrm{d} \boldsymbol{c}_{i}$, $i \in \mathfrak{S}$, using the expression of the right hand sides (4.26), and using standard kinetic 
theory techniques, it is obtained that

$$
\mathcal{V}_{i}=-\sum_{j \in \mathfrak{S}} \frac{1}{3} k_{\mathrm{B}} T\left[\phi^{D_{i}}, \phi^{D_{j}}\right] \boldsymbol{X}_{j}^{\mathrm{th}}-\frac{1}{3} k_{\mathrm{B}} T\left[\phi^{D_{i}}, \phi^{\lambda}\right] \nabla\left(\frac{-1}{k_{\mathrm{B}} T}\right),
$$

where for any $\xi=\left(\xi_{i}\right)_{i \in \mathfrak{S}}$ and $\zeta=\left(\zeta_{i}\right)_{i \in \mathfrak{S}}$, with $\xi_{i}$ and $\zeta_{i}$ functions of $\boldsymbol{c}_{i}$, the bracket $[\xi, \zeta]$ reads

$$
[\xi, \zeta]=\left\langle\left\langle f^{(0)} \xi, \mathcal{I} \zeta\right\rangle\right\rangle=\left\langle\left\langle f^{(0)} \mathcal{I} \xi, \zeta\right\rangle\right\rangle
$$

with $\mathcal{I} \zeta=\left(\mathcal{I}_{i}(\zeta)\right)_{i \in \mathfrak{S}}, f^{(0)} \xi=\left(f_{i}^{(0)} \xi_{i}\right)_{i \in \mathfrak{S}}$, and the scalar product $\langle\langle\xi, \zeta\rangle\rangle$ is given by

$$
\langle\langle\xi, \zeta\rangle\rangle=\sum_{i \in \mathfrak{S}} \int \xi_{i} \odot \zeta_{i} \mathrm{~d} \boldsymbol{c}_{i},
$$

with $\odot$ denoting the tensor maximum contraction symbol. The expressions (4.38) are then directly obtained upon letting

$$
\begin{gathered}
L_{i j}=\frac{1}{3} \rho_{i} \rho_{j} k_{\mathrm{B}} T^{2}\left[\phi^{D_{i}}, \phi^{D_{j}}\right], \quad i, j \in \mathfrak{S}, \\
L_{i \mathrm{e}}=\frac{1}{3} \rho_{i} T\left[\phi^{D_{i}}, \phi^{\widehat{\lambda}}\right], \quad i \in \mathfrak{S} .
\end{gathered}
$$

The resulting expressions for transport coefficients in dense gases are generally highly complex and we refer to the literature for more details [50]. The symmetry relations have notably been investigated by Ernst [80], including triple collisions.

The coefficients $L=\left(L_{i j}\right)_{i, j \in \mathfrak{S} \cup\{\mathrm{e}\}}$ are usually termed phenomenological coefficients in the thermodynamic literature but are not anymore phenomenological when obtained from the kinetic theory of dense gases and the link with the traditional transport coefficients is addressed in the next section.

\subsubsection{Dissipative fluxes with thermodynamic stability}

We discuss in this section the link between the matrix $L$ and the traditional fluid mixture transport coefficients. In order to define the standard diffusion coefficients, it is necessary to subtract a temperature gradient from the forces $\boldsymbol{X}_{i}^{\text {th }}, i \in \mathfrak{S}$, and this procedure traditionally involves pressure based thermodynamic property. The traditional transport coefficients thus require the mechanical stability condition $\partial_{\rho} p<0$.

In the situation of mechanical stability, the map $\left(T, \rho, \mathrm{y}_{1}, \ldots, \mathrm{y}_{\mathrm{n}_{\mathrm{s}}}\right) \mapsto\left(T, p, \mathrm{y}_{1}, \ldots, \mathrm{y}_{\mathrm{n}_{\mathrm{s}}}\right)$ is locally invertible and one may introduce thermodynamic properties as functions of $\left(T, p, \mathrm{y}_{1}, \ldots, \mathrm{y}_{\mathrm{n}_{\mathrm{s}}}\right)$. For any thermodynamic function $\overline{\mathcal{A}}^{\mathrm{u}}\left(T, \rho, \mathrm{y}_{1}, \ldots, \mathrm{y}_{\mathrm{n}_{\mathrm{s}}}\right)$ we may then define the pressure based thermodynamic property

$$
\widetilde{\mathcal{A}}^{\mathrm{u}}\left(T, p, \mathrm{y}_{1}, \ldots, \mathrm{y}_{\mathrm{s}}\right)=\overline{\mathcal{A}}^{\mathrm{u}}\left(T, \rho, \mathrm{y}_{1}, \ldots, \mathrm{y}_{\mathrm{n}_{\mathrm{s}}}\right),
$$

and the pressure-based Gibbs relation is in the form

$$
\mathrm{d} \widetilde{g}^{\mathrm{u}}=\widetilde{\nu}^{\mathrm{u}} \mathrm{d} p^{\mathrm{u}}-\widetilde{s}^{\mathrm{u}} \mathrm{d} T+\sum_{k \in \mathfrak{S}} \widetilde{g}_{k}^{\mathrm{u}} \mathrm{dy} y_{k}
$$

Letting then for any thermodynamic property $\mathcal{A}^{\mathrm{u}}$

$$
\widetilde{\mathcal{A}}_{k}^{\mathrm{u}}=\partial_{\mathrm{y}_{k}} \widetilde{\mathcal{A}}^{\mathrm{u}}\left(T, p, \mathrm{y}_{1}, \ldots, \mathrm{y}_{\mathrm{n}_{\mathrm{s}}}\right),
$$


we recover from (4.45) that the Gibbs functions $\bar{g}_{k}^{\mathrm{u}}, k \in \mathfrak{S}$, are naturally pressure based $\widetilde{g}_{k}^{\mathrm{u}}=\bar{g}_{k}, k \in \mathfrak{S}$. We also obtain from (4.45) after some calculus

$$
\mathrm{d}\left(\frac{\widetilde{g}_{k}^{\mathrm{u}}}{T}\right)=\frac{\widetilde{\nu}_{k}^{\mathrm{u}}}{T} \mathrm{~d} p-\frac{\widetilde{h}_{k}^{\mathrm{u}}}{T^{2}} \mathrm{~d} T+\sum_{l \in \mathfrak{S}} \frac{\partial_{\mathrm{y}_{l}} \widetilde{g}_{k}^{\mathrm{u}}}{T} \mathrm{dy}_{l},
$$

where $\widetilde{h}_{k}^{\mathrm{u}}=\partial_{\mathrm{y}_{k}} h^{\mathrm{u}}\left(T, p, \mathrm{y}_{1}, \ldots, \mathrm{y}_{\mathrm{n}_{\mathrm{s}}}\right)$ is the enthalpy of the $k$ th species, $h^{\mathrm{u}}=e^{\mathrm{u}}+p^{\mathrm{u}} / \rho$ the mixture enthalpy per unit mass and $\rho h^{\mathrm{u}}=\mathcal{H}^{\mathrm{u}}=\mathcal{E}^{\mathrm{u}}+p^{\mathrm{u}}$. The classical diffusion driving forces $\boldsymbol{d}_{i}^{\mathrm{cl}}, i \in \mathfrak{S}$, are then obtained by subtracting from the thermodynamic diffusion driving forces $\boldsymbol{d}_{i}^{\text {th }}=\mathrm{x}_{i} m_{i} \boldsymbol{\nabla}\left(\bar{g}^{\mathrm{u}} / k_{\mathrm{B}} T\right)$ the proper temperature gradient terms

$$
\boldsymbol{d}_{i}^{\mathrm{cl}}=\boldsymbol{d}_{i}^{\mathrm{th}}+\frac{\mathrm{x}_{i} m_{i} \widetilde{h}_{i}^{\mathrm{u}}}{k_{\mathrm{B}} T^{2}} \nabla T, \quad i \in \mathfrak{S} .
$$

The resulting traditional diffusion driving forces $\boldsymbol{d}_{i}^{\mathrm{cl}}$ are then linear combinations of mole fractions and pressure gradients.

In order to relate the mass and heat transport coefficients $L_{i j}, i, j \in \mathfrak{S} \cup\{\mathrm{e}\}$, to the traditional transport coefficients, we denote by $L$ the matrix

$$
L=\left(\begin{array}{cccc}
L_{11} & \cdots & L_{1 \mathrm{n}_{\mathrm{s}}} & L_{1 \mathrm{e}} \\
\vdots & \ddots & \vdots & \vdots \\
L_{\mathrm{n}_{\mathrm{s}} 1} & \cdots & L_{\mathrm{n}_{\mathrm{s}} \mathrm{n}_{\mathrm{s}}} & L_{\mathrm{n}_{\mathrm{s}} \mathrm{e}} \\
L_{\mathrm{e} 1} & \cdots & L_{\mathrm{en}_{\mathrm{s}}} & L_{\mathrm{ee}}
\end{array}\right)
$$

The matrix $L$ is symmetric positive semi-definite and has a one-dimensional nullspace spanned by $(0,1, \ldots, 1)^{t}$. We introduce then the modified matrix $\widehat{L}=A^{t} L A$ with

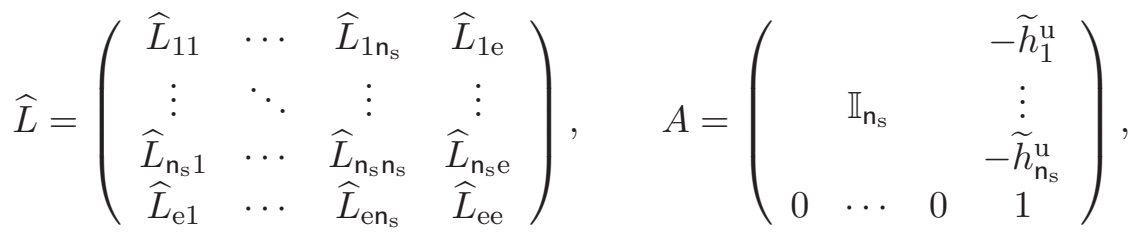

where $\mathbb{I}_{\mathrm{n}_{\mathrm{s}}}$ denotes the identity matrix in $\mathbb{R}^{\mathrm{n}_{\mathrm{s}}}$. The high pressure multicomponent diffusion coefficients $D_{i j}, i, j \in \mathfrak{S}$, thermal diffusion coefficients $D_{i}^{\mathrm{TD}}, i \in \mathfrak{S}$, and partial thermal conductivity $\widehat{\lambda}$ are next defined by $[81,60]$

$$
D_{i j}=\frac{n k_{\mathrm{B}} \widehat{L}_{i j}}{\rho_{i} \rho_{j}}, \quad i, j \in \mathfrak{S}, \quad D_{i}^{\mathrm{TD}}=\frac{\widehat{L}_{i \mathrm{e}}}{\rho_{i} T}=\frac{\widehat{L}_{\mathrm{e} i}}{\rho_{i} T}, \quad i \in \mathfrak{S}, \quad \widehat{\lambda}=\frac{\widehat{L}_{\mathrm{ee}}}{T^{2}} .
$$

Using the high pressure coefficients (4.49) and the traditional diffusion driving forces (4.46), the fluxes $\overline{\mathcal{F}}_{i}, i \in \mathfrak{S}$, and $\boldsymbol{Q}$ may be rewritten in the more familiar form

$$
\begin{aligned}
\overline{\mathcal{F}}_{i} & =-\sum_{j \in \mathfrak{S}} \rho_{i} D_{i j} \boldsymbol{d}_{j}^{\mathrm{cl}}-\rho_{i} D_{i}^{\mathrm{TD}} \nabla \ln T, \quad i \in \mathfrak{S}, \\
\boldsymbol{Q} & =-n k_{\mathrm{B}} T \sum_{j \in \mathfrak{S}} \theta_{j} \boldsymbol{d}_{j}^{\mathrm{cl}}-\widehat{\lambda} \boldsymbol{\nabla} T+\sum_{i \in \mathfrak{S}} \widetilde{h}_{i}^{\mathrm{u}} \mathcal{F}_{i},
\end{aligned}
$$

where $D_{i j}$ are the symmetric multicomponent diffusion coefficients, $D_{i}^{\mathrm{TD}}$ the thermal diffusion coefficients, and $\widehat{\lambda}$ the partial thermalconductivity. The symmetric multicomponent diffusion coefficients $D_{i j}$ have notably been considered by Kurochkin et al. [81] and generalize the coefficients introduced for dilute gases by Waldmann [82, 83, 56]. 


\subsection{Van der Waals equation of state}

In order to recover the van der Waals equation of state, we may assume that the interaction potentials $\varphi_{i j}$ are such that

$$
\varphi_{i j}\left(r_{i j}\right)= \begin{cases}+\infty & \text { if } 0 \leq r_{i j} \leq \sigma_{i j} \\ \varphi_{i j}\left(r_{i j}\right)<\infty & \text { if } \sigma_{i j}<r_{i j}\end{cases}
$$

for some positive collision diameters $\sigma_{i j}$ depending on the species pairs $(i, j)$. One may also assume that $\varphi_{i j}$ is attractive $\varphi_{i j}<0$ for $2 \sigma_{i j}<r_{i j}$, and increases towards zero as $r_{i j} \rightarrow \infty$. One may then recover the van der Waals equation of state by decomposing the integrals $2 \pi \int \mathfrak{f}_{i j} \mathrm{~d} r_{i j}$ in two zones and proceeding as for single species fluids [84, 85, 86]. More specifically, one may write that

$$
\int \mathfrak{f}_{i j} r_{i j}^{2} \mathrm{~d} r_{i j}=\int_{0}^{2 \sigma_{i j}} \mathfrak{f}_{i j} r_{i j}^{2} \mathrm{~d} r_{i j}+\int_{2 \sigma_{i j}}^{\infty} \mathfrak{f}_{i j} r_{i j}^{2} \mathrm{~d} r_{i j}
$$

use $\mathfrak{f}_{i j}=-1$ over $\left(0,2 \sigma_{i j}\right)$, linearize the Mayer functions $\mathfrak{f}_{i j} \approx-\varphi_{i j} / k_{\mathrm{B}} T$ for $2 \sigma_{i j}<r_{i j}$, to get that

$$
2 \pi k_{\mathrm{B}} T \int \mathfrak{f}_{i j} \mathrm{~d} r_{i j} \approx-4 k_{\mathrm{B}} T \frac{4 \pi \sigma_{i j}^{3}}{3}-2 \pi \int_{2 \sigma_{i j}}^{\infty} \varphi_{i j}\left(\mathbf{r}_{i j}\right) r_{i j}^{2} \mathrm{~d} r_{i j}
$$

and the corresponding pressure law reads

$$
p^{\mathrm{u}}=\sum_{i \in \mathfrak{S}} n_{i} k_{\mathrm{B}} T+\sum_{i, j \in \mathfrak{S}} n_{i} n_{j} k_{\mathrm{B}} T \frac{16 \pi \sigma_{i j}^{3}}{3}+\sum_{i, j \in \mathfrak{S}} n_{i} n_{j} 2 \pi \int_{2 \sigma_{i j}}^{\infty} \varphi_{i j}\left(\mathbf{r}_{i j}\right) r_{i j}^{2} \mathrm{~d} r_{i j} .
$$

Assuming naturally that $\sigma_{i j}$ is in the form $\sigma_{i j}=\frac{1}{2}\left(\sigma_{i}+\sigma_{j}\right), i, j \in \mathfrak{S}$, where $\sigma_{i}$ is a typical collision diameter of the $i$ th species, the sum $\sum_{i, j \in \mathfrak{S}} n_{i} n_{j} \sigma_{i j}^{3}$ may be rewritten $\sum_{i, j \in \mathfrak{S}} \frac{1}{4} n_{i} n_{j}\left(\sigma_{j}^{3}+3 \sigma_{j}^{2} \sigma_{i}\right)$ and approximated as $\sum_{i, j \in \mathfrak{S}} n_{i} n_{j} \sigma_{j}^{3}$. Assuming then that the volume occupied by the particules is small

$$
\sum_{j \in \mathfrak{S}} n_{j} \frac{4 \pi \sigma_{j}^{3}}{3}<<1
$$

we obtain the state law

$$
p^{\mathrm{u}}=\sum_{i \in \mathfrak{S}} \frac{n_{i} k_{\mathrm{B}} T}{1-\sum_{j \in \mathfrak{S}} \mathrm{b}_{j} n_{j}}-\sum_{i, j \in \mathfrak{S}} n_{i} n_{j} \mathrm{a}_{i j}
$$

where

$$
\mathrm{b}_{j}=4 \frac{4 \pi \sigma_{j}^{3}}{3}, \quad \mathrm{a}_{i j}=-2 \pi \int_{2 \sigma_{i j}}^{\infty} \varphi_{i j}\left(\mathbf{r}_{i j}\right) r_{i j}^{2} \mathrm{~d} r_{i j}
$$

and the van der Waals equation of state for mixtures has been recovered from (4.10) in a similar way as in statistical physics $[84,85,86]$. Moreover, we have also obtained from (4.10) the traditional mixing rules for the covolume $\sum_{j \in \mathfrak{S}} \mathrm{b}_{j} n_{j}$ as well as for the attractive term $\sum_{i, j \in \mathfrak{S}} n_{i} n_{j} \mathrm{a}_{i j}$ introduced by Berthelot [87]. 


\subsection{Orders of magnitude}

We estimate in this section the order of magnitude of nonideal terms in thermodynamic functions. To this aim, we introduce a characteristic temperature $T^{\star}$, a characteristic interparticle distance $r^{\star}$, and a characteristic particle mass $m^{\star}$. The characteristic number density is then $n^{\star}=1 / r^{\star 3}$ and the particle thermal velocity $c^{\star}$ such that $m^{\star} c^{\star 2}=k_{\mathrm{B}} T^{\star}$. We also denote by $\tau_{\mathrm{K}}^{\star}$ a characteristic collision time, by $l_{\mathrm{K}}^{\star}=\tau_{\mathrm{K}}^{\star} c^{\star}$ the mean free path, by $\tau_{\mathrm{H}}^{\star}$ a characteristic hydrodynamic time or fluid time and by $l_{\mathrm{H}}^{\star}=\tau_{\mathrm{H}}^{\star} c^{\star}$ the corresponding fluid length. We further introduce a characteristic collision diameter $\sigma^{\star}$, also typical of the range of the interaction potentials, and we generally have $\sigma^{\star} \ll r^{\star} \ll l_{\mathrm{K}}^{\star} \ll l_{\mathrm{H}}^{\star}$ as well as $\sigma^{\star 2} l_{\mathrm{K}}^{\star}=r^{\star 3}$ and the Knudsen number is given by $l_{\mathrm{K}}^{\star} / l_{\mathrm{H}}^{\star}$.

From the expression (4.12) of the second virial coefficients $\beta_{i j}, i, j \in \mathfrak{S}$, we then obtain that $\beta^{\star}=\beta_{i j}^{\star}=\sigma^{\star 3}$. We may next estimate that the nonideal terms in the pressure (4.13) are typically of the order $k_{\mathrm{B}} T^{\star} \sigma^{\star 3} / r^{\star 6}$. The ratio of the nonideal terms $\sum_{i, j \in \mathfrak{S}} \frac{1}{2} n_{i} n_{j} \beta_{i j} k_{\mathrm{B}} T$ to the ideal terms $\sum_{i \in \mathfrak{S}} n_{i} k_{\mathrm{B}} T$ may then be estimated to be of the order $\left(\sigma^{\star} / r^{\star}\right)^{3}$ as discussed notably by landau, Lifchitz and Pitaevskii [85, 70]. The nonideal to ideal term ratio is thus usually modest but increase with the number density $n^{\star}=1 / r^{\star 3}$ and is ultimately of order unity for liquid like states where the interparticle distance $r^{\star}$ is of the order of the collision diameter $\sigma^{\star}$.

\section{$5 \quad$ Euler/van-der-Waals fluid equations}

We derive in this section the Euler/van der Waals fluid equations at zeroth order of Enskog expansion. The method combines symmetrized zeroth order pair distribution functions as well as higher order Taylor expansions of pair distribution functions.

\subsection{The symmetrized pair correlation functions}

Previous work concerning single species fluids has shown that higher order expansions of pair distribution functions are required in order to derive diffuse interface models [15]. It is thus important to select proper pair distribution functions before using Taylor expansions.

The zeroth order pair distribution functions (4.5) of Section 4.2 have traditionally been used in the literature for investigating gas mixtures $[49,50,51]$ as well as for investigating single species fluids $[52,56,57,58,54]$. When substituted in the governing equations and when using first order Taylor expansions, the nonideal fluid equations with normal diffusive processes [50], presented in Section 4, are obtained. However, these pair distribution functions $f_{i j}^{(0), c l}$ are not without criticism. A first point is that $f_{i j}^{(0), c l}$ is not symmetric in its arguments as it should be for a pair distribution function. Moreover, when $r_{i j}$ is large, $g_{i j}\left(\mathbf{r}_{i}, r_{i j}\right)$ becomes unity and $f_{i j}^{(0), c l}$ reduces to $f_{i}^{(0)}\left(\mathbf{r}_{i}, \boldsymbol{c}_{i}, t\right) f_{j}^{(0)}\left(\mathbf{r}_{i}, \boldsymbol{c}_{j}, t\right)$ that has no physical meaning.

In this section, instead of using the distributions $f_{i j}^{(0), c l}$, the following symmetrized pair distribution functions $f_{i j}^{(0), \text { sy }}$ are used [15]

$$
f_{i j}^{(0), \mathrm{sy}}=f_{i}^{(0)}\left(\mathbf{r}_{i}, \boldsymbol{c}_{i}, t\right) f_{j}^{(0)}\left(\mathbf{r}_{j}, \boldsymbol{c}_{j}, t\right) g_{i j}\left(\overline{\mathbf{r}}_{i, j}, r_{i j}\right), \quad i, j \in \mathfrak{S},
$$

where $\overline{\mathbf{r}}_{i, j}$ denotes a symmetric average of $\mathbf{r}_{i}$ and $\mathbf{r}_{j}$ like the center of mass $\overline{\mathbf{r}}_{i, j}=\mu_{i} \mathbf{r}_{i}+\mu_{j} \mathbf{r}_{j}$ or the midpoint $\frac{1}{2}\left(\mathbf{r}_{i}+\mathbf{r}_{j}\right)$. We first note that $f_{i j}^{(0) \text {,sy }}$ and $f_{i j}^{(0), \text { cl }}$ coincide at zeroth order when $\mathbf{r}_{i}=\mathbf{r}_{j}=\overline{\mathbf{r}}_{i, j}$ so that $f_{i j}^{(0) \text {,sy }}$ is also in the kernel of the operator $\mathcal{J}_{i}^{(0)}$ that only 
consider the values of $f_{i j}$ on the linear subspace $\mathbf{r}_{i}=\mathbf{r}_{j}$. Moreover, $f_{i j}^{(0) \text {,sy }}$ is symmetric in its argument and the limit for large $r_{i j}$ is the natural uncorrelated pair distribution function $f_{i}^{(0)}\left(\mathbf{r}_{i}, \boldsymbol{c}_{i}, t\right) f_{i}^{(0)}\left(\mathbf{r}_{j}, \boldsymbol{c}_{j}, t\right)$. Previous work has also shown that diffuse interface fluid models may be recovered from such symmetrized distributions in the situation of single species fluids [15]. In order to derive the macroscopic equations, the symmetrized zeroth order pair distribution functions $f_{i j}^{(0) \text {,sy }}$ are thus used with the general balance equations established in Section 3.2 as well as with higher order Taylor expansions of pair distribution functions.

Although a nonequilibrium correlation function $g_{i j}$ could in principle also be used, we will nevertheless use a correlation function (4.6) in the simplified form $g_{i j}\left(\mathbf{r}_{i}, r_{i j}\right)$ as if the temperature were locally uniform. There is indeed no clear expression for a nonequilibrium correlation function at interfaces and many solutions have been proposed like using various average temperatures, or the center of mass temperature, or else solving integral equations $[3,88,89]$. In addition, temperature variations during phase change are modest, keeping in mind that equilibrium fronts are isotherms, at variance with large density variations. Therefore, using either $\mathbf{r}_{i}$ or a symmetric average $\overline{\mathbf{r}}_{i, j}$ would yield the same results and we will frequently denote by $g_{i j}\left(r_{i j}\right)$ the corresponding function, the dependence on $\mathbf{r}_{i}$ being left implicit. Using a locally uniform correlation function $g_{i j}$ will imply that the capillary coefficients are naturally independent of temperature in agreement with the van der Waals and Rayleigh approximate expressions.

In summary, we have to revisit the Chapman-Enskog method for mixtures of dense gases using the Enskog scaling (4.1), the zeroth pair distribution function (5.1), as well as higher order Taylor expansions of pair distribution functions, following the derivation of single species diffuse interface fluid equations [15]. Since new thermodynamic properties depending on derivatives will be obtained, we keep the superscript ${ }^{\mathrm{u}}$ to denote the nonideal bulk phase thermodynamic properties obtained in Section 4 that do not involve gradients. As a general result, when pair distribution functions are expanded in the potential parts of the internal energy and the pressure tensor, nonideal terms are obtained at zeroth order whereas diffuse interface terms involving density gradients are obtained at second order.

\subsection{A generalized Korteweg tensor}

We consider the tensor $\mathcal{P}^{\mathrm{P}}$ arising from interaction potentials (3.15) and look for extra terms with two spatial derivatives $\mathcal{P}^{\mathrm{ex}}$ arising from second order expansions of the distribution functions $f_{i j}^{(0), \text { sy }}\left(\mathbf{r}_{i}, \boldsymbol{c}_{i}, \mathbf{r}_{j}, \boldsymbol{c}_{j}, t\right)=f_{i}^{(0)}\left(\mathbf{r}_{i}, \boldsymbol{c}_{i}, t\right) f_{j}^{(0)}\left(\mathbf{r}_{j}, \boldsymbol{c}_{j}, t\right) g_{i j}\left(r_{i j}\right)$. We use Taylor expansions of $f_{i j}^{(0) \text {,sy }}$ and only keep the extra terms involving two derivatives.

The relevant integrand factors in (3.15) may first be expanded in the form

$$
\begin{aligned}
n_{i j}\left(\mathbf{r}_{i}-\right. & \left.(1-\alpha) \mathbf{r}_{i j}, \mathbf{r}_{i}+\alpha \mathbf{r}_{i j}, t\right) \approx \\
& \left(n_{i}\left(\mathbf{r}_{i}\right)-(1-\alpha) \boldsymbol{\nabla} n_{i}\left(\mathbf{r}_{i}\right) \cdot \mathbf{r}_{i j}+\frac{1}{2}(1-\alpha)^{2} \boldsymbol{\nabla}^{2} n_{i}\left(\mathbf{r}_{i}\right):\left(\mathbf{r}_{i j} \otimes \mathbf{r}_{i j}\right)\right) \\
& \times\left(n_{j}\left(\mathbf{r}_{i}\right)+\alpha \boldsymbol{\nabla} n_{j}\left(\mathbf{r}_{i}\right) \cdot \mathbf{r}_{i j}+\frac{1}{2} \alpha^{2} \nabla^{2} n_{j}\left(\mathbf{r}_{i}\right):\left(\mathbf{r}_{i j} \otimes \mathbf{r}_{i j}\right)\right) \times g_{i j}\left(r_{i j}\right),
\end{aligned}
$$

where the dependencies on time $t$ have been left implicit. Extracting the terms with two derivatives, the relevant integrands involve the factors

$$
-\alpha(1-\alpha) \nabla n_{i}\left(\mathbf{r}_{i}\right) \cdot \mathbf{r}_{i j} \nabla n_{j}\left(\mathbf{r}_{i}\right) \cdot \mathbf{r}_{i j}
$$

and

$$
\frac{1}{2}(1-\alpha)^{2} n_{j}\left(\mathbf{r}_{i}\right) \nabla^{2} n_{i}\left(\mathbf{r}_{i}\right):\left(\mathbf{r}_{i j} \otimes \mathbf{r}_{i j}\right)+\frac{1}{2} \alpha^{2} n_{i}\left(\mathbf{r}_{i}\right) \nabla^{2} n_{j}\left(\mathbf{r}_{i}\right):\left(\mathbf{r}_{i j} \otimes \mathbf{r}_{i j}\right) .
$$


Incidentally, the products $n_{i}\left(\mathbf{r}_{i}\right) n_{j}\left(\mathbf{r}_{i}\right)$ are already taken into account in the state law (4.13) whereas odd terms yield zero contributions. Odd terms only contribute in first order linearized equations, that is, in the dissipative fluxes [57, 54, 50].

Focusing on the first integrands, we note that

$$
\boldsymbol{\nabla} n_{i}\left(\mathbf{r}_{i}\right) \cdot \mathbf{r}_{i j} \boldsymbol{\nabla} n_{j}\left(\mathbf{r}_{i}\right) \cdot \mathbf{r}_{i j}=\nabla n_{i}\left(\mathbf{r}_{i}\right) \otimes \nabla n_{j}\left(\mathbf{r}_{i}\right):\left(\mathbf{r}_{i j} \otimes \mathbf{r}_{i j}\right),
$$

and using $\int_{0}^{1} \alpha(1-\alpha) \mathrm{d} \alpha=\frac{1}{6}$ we obtain a first extra contribution $\mathfrak{p}_{1}$ to the pressure tensor in the form

$$
\mathfrak{p}_{1}=\sum_{i, j \in \mathfrak{S}} \frac{1}{12} \int \frac{\varphi_{i j}^{\prime}\left(r_{i j}\right)}{r_{i j}} g_{i j}\left(r_{i j}\right) \mathbf{r}_{i j} \otimes \mathbf{r}_{i j} \nabla n_{i} \otimes \nabla n_{j}:\left(\mathbf{r}_{i j} \otimes \mathbf{r}_{i j}\right) \mathrm{d} \mathbf{r}_{i j}
$$

We may now use the identity (F.3) from Appendix F in order to perform the integrations over $\mathbf{r}_{i j} \in \mathbb{R}^{3}$, and, using elementary symmetries, we obtain that

$$
\mathfrak{p}_{1}=\sum_{i, j \in \mathfrak{S}} \frac{\varkappa_{i j}}{6}\left(2 \nabla n_{i} \otimes \nabla n_{j}+\nabla n_{i} \cdot \nabla n_{j} \boldsymbol{I}\right),
$$

where the diffuse interface or capillarity coefficients $\varkappa_{i j}$ are defined by

$$
\varkappa_{i j}=\frac{1}{30} \int \varphi_{i j}^{\prime}\left(r_{i j}\right) g_{i j}\left(r_{i j}\right) r_{i j}^{3} \mathrm{~d} \mathbf{r}_{i j}=\frac{2 \pi}{15} \int \varphi_{i j}^{\prime}\left(r_{i j}\right) g_{i j}\left(r_{i j}\right) r_{i j}^{5} \mathrm{~d} r_{i j}
$$

Focusing next on integrands from the Hessian matrices $\nabla^{2} n_{i}$, using $\int_{0}^{1} \alpha^{2} \mathrm{~d} \alpha=\int_{0}^{1}(1-$ $\alpha)^{2} \mathrm{~d} \alpha=\frac{1}{3}$, and using elementary symmetries, we obtain a second extra contribution $\mathfrak{p}_{2}$ to the pressure tensor in the form

$$
\mathfrak{p}_{2}=-\sum_{i, j \in \mathfrak{S}} \frac{n_{i}}{6} \int \frac{\varphi_{i j}^{\prime}\left(r_{i j}\right)}{r_{i j}} g_{i j}\left(r_{i j}\right) \mathbf{r}_{i j} \otimes \mathbf{r}_{i j} \nabla^{2} n_{j}:\left(\mathbf{r}_{i j} \otimes \mathbf{r}_{i j}\right) \mathrm{d} \mathbf{r}_{i j}
$$

Using again the identity (F.3) in order to perform the integrations over $\mathbf{r}_{i j} \in \mathbb{R}^{3}$, and using elementary symmetries, we obtain that

$$
\mathfrak{p}_{2}=-\sum_{i, j \in \mathfrak{S}} \frac{\varkappa_{i j}}{3}\left(2 n_{i} \nabla^{2} n_{j}+n_{i} \Delta n_{j} \boldsymbol{I}\right)
$$

Collecting previous results, the extra pressure tensor $\mathcal{P}^{\text {ex }}$ is found in the form

$$
\mathcal{P}^{\mathrm{ex}}=\sum_{i, j \in \mathfrak{S}} \frac{\varkappa_{i j}}{6}\left(2 \nabla n_{i} \otimes \nabla n_{j}+\nabla n_{i} \cdot \nabla n_{j} \boldsymbol{I}-4 n_{i} \nabla^{2} n_{j}-2 n_{i} \Delta n_{j} \boldsymbol{I}\right) .
$$

We may then simplify $\mathcal{P}^{\text {ex }}$ with an equivalent expression $\overline{\mathcal{P}}^{\text {ex }}$, that is, we seek $\overline{\mathcal{P}}^{\text {ex }}$ such that

$$
\nabla \cdot \mathcal{P}^{\mathrm{ex}}=\nabla \cdot \overline{\mathcal{P}}^{\mathrm{ex}}
$$

Expressing the divergence of the Hessian matrices contributions $n_{i} \nabla^{2} n_{j}$ with the help of differential identity (F.7), we indeed obtain the simplified pressure tensor

$$
\overline{\mathcal{P}}^{\mathrm{ex}}=\sum_{i, j \in \mathfrak{S}} \varkappa_{i j}\left(\nabla n_{i} \otimes \nabla n_{j}-\frac{1}{2} \nabla n_{i} \cdot \nabla n_{j} \boldsymbol{I}-n_{i} \Delta n_{j} \boldsymbol{I}\right) .
$$


Introducing the mass based diffuse interface coefficient $\bar{\varkappa}_{i j}=\varkappa_{i j} / m_{i} m_{j}$ and using $\rho_{i}=$ $m_{i} n_{i}$ we may also rewrite the extra pressure tensor $\boldsymbol{\nabla} \cdot \overline{\mathcal{P}}^{\mathrm{ex}}$ as

$$
\overline{\mathcal{P}}^{\mathrm{ex}}=\sum_{i, j \in \mathfrak{S}} \bar{\varkappa}_{i j}\left(\nabla \rho_{i} \otimes \nabla \rho_{j}-\frac{1}{2} \nabla \rho_{i} \cdot \nabla \rho_{j} \boldsymbol{I}-\rho_{i} \Delta \rho_{j} \boldsymbol{I}\right),
$$

that coincides with the generalized Korteweg tensor $\overline{\mathcal{P}}^{\mathrm{ex}}=\mathcal{P}^{\text {KO }}$ given in $(2.18)$ and derived from rational thermodynamics in the simplified situation of constant diffuse interface coefficients $\varkappa_{i j}, i, j \in \mathfrak{S}$. This tensor also coincide with the traditional Korteweg tensor derived for single species fluids [15].

\subsection{Diffuse interface coefficients}

The diffuse interface or capillary coefficients $\varkappa_{i j}$ have been found in the form (5.2) where $g_{i j}\left(r_{i j}\right)=\exp \left(-\varphi_{i j}\left(r_{i j}\right) / k_{\mathrm{B}} T\left(\mathbf{r}_{i}\right)\right)$. We may integrate by part the integral in (5.2) to get that

$$
\varkappa_{i j}=\frac{1}{6} k_{\mathrm{B}} T \int \mathfrak{f}_{i j} r_{i j}^{2} \mathrm{~d} \mathbf{r}_{i j}=\frac{2 \pi}{3} k_{\mathrm{B}} T \int \mathfrak{f}_{i j} r_{i j}^{4} \mathrm{~d} r_{i j},
$$

where $\mathfrak{f}_{i j}$ is the Mayer function (4.11) of the $(i, j)$ th species pair. The zeroth moments of the Mayer functions thus yield the second virial coefficients $\beta_{i j}$ whereas the second moments yield the diffuse interface coefficients $\varkappa_{i j}, i, j \in \mathfrak{S}$, up to the $\frac{1}{6} k_{\mathrm{B}} T$ factor. A notable property of the diffuse interface coefficients $\varkappa_{i j}$ given by (5.2) is that they are independent of the species number densities $n_{k}, k \in \mathfrak{S}$.

We may also simplify (5.5) in order to recover the van der Waals and Rayleigh capillarity coefficient. To this aim, we assume that the interaction potentials are like (4.52). We may simplify then the integral in (5.5) by neglecting the integrals over $\left(0, \sigma_{i j}\right)$ and by linearizing $\mathfrak{f}_{i j}$ in the form $\mathfrak{f}_{i j} \approx-\varphi_{i j} / k_{\mathrm{B}} T$ for $r_{i j}>\sigma_{i j}$, and this yields

$$
\varkappa_{i j}=-\frac{1}{6} \int_{r_{i j}>\sigma_{i j}} \varphi_{i j} r_{i j}^{2} \mathrm{~d} \mathbf{r}_{i j}, \quad i, j \in \mathfrak{S},
$$

that coincide with the van der Waals and Rayleigh formula for single species fluids [3].

The van der Waals and Rayleigh simplified capillarity coefficients (5.6) are then independent of species number densities and temperature. In a kinetic framework, the independence on temperature notably requires to neglect temperature derivative contributions of capillarity that would arise through the correlation function $g_{i j}$, and that are typically in the form $\int \varphi_{i j}^{\prime}\left(r_{i j}\right) \varphi_{i j}^{k}\left(r_{i j}\right) \exp \left(-\frac{\varphi_{i j}}{k_{\mathrm{B}} T}\right) r_{i j}^{3} \mathrm{~d} \mathbf{r}_{i j}$ where $k \geq 1$ is an integer. We will assume in the kinetic derivation of Cahn-Hilliard equations that the diffuse interface coefficients are independent of temperature for the sake of simplicity.

We further deduce from the expression (5.5) that the diffuse interface coefficients $\varkappa_{i j}$ are of the order $\varkappa^{\star}=\varkappa_{i j}^{\star}=k_{\mathrm{B}} T^{\star} \sigma^{\star 5}$ and thus vary like the fifth power of the collision diameter $\sigma^{\star}$ wheras the second virial coefficients scale as $\beta^{\star}=\sigma^{\star 3}$. We may thus estimate that the diffuse interface terms in the pressure (5.3) are typically of the order $\varkappa^{\star}(\nabla n)^{\star 2}=k_{\mathrm{B}} T^{\star} n^{\star}\left(\frac{\sigma^{\star}}{r^{\star}}\right)^{3}\left(\frac{\sigma^{\star}}{l_{\nabla n}^{\star}}\right)^{2}$ where $l_{\nabla n}^{\star}$ is a characteristic length typical of density gradients. The ratio of the diffuse interface terms $\sum_{i, j \in \mathfrak{S}} \varkappa_{i j} \boldsymbol{\nabla} n_{i} \otimes \nabla n_{j}$ to the ideal terms $\sum_{i \in \mathfrak{S}} n_{i} k_{\mathrm{B}} T$ may then be estimated to be

$$
\left(\frac{\sigma^{\star}}{r^{\star}}\right)^{3}\left(\frac{\sigma^{\star}}{l_{\nabla n}^{\star}}\right)^{2}
$$


The capillary terms are thus generally very small unless the interparticle distance $\mathrm{r}^{\star}$ is becoming close to the collision diameter $\sigma^{\star}$, i.e., within a liquid-like fluid, and, simultaneously, the density gradients length $l_{\nabla n}^{\star}$ is also becoming close to $\sigma^{\star}$, i.e., in a phase change interface like a vaporization front.

\subsection{The energy density}

We have to investigate the extra terms $\mathcal{E}^{\text {ex }}$ arising from the energy density $\mathcal{E}^{\mathrm{P}}$ of potential origin (3.9) using the pair distribution functions $f_{i j}^{(0), s y}$. Expanding the zeroth order twoparticle distribution functions $f_{i j}^{(0) \text {,sy }}=f_{i}\left(\boldsymbol{x}_{i}, t\right) f_{i}\left(\boldsymbol{x}_{j}, t\right) g_{i j}\left(r_{i j}\right)$ around $\mathbf{r}_{j}=\mathbf{r}_{i}+\mathbf{r}_{i j}$, the relevant extra terms involving two derivatives are in the form

$$
\frac{1}{2} \int \varphi_{i j}\left(r_{i j}\right) g_{i j}\left(r_{i j}\right) n_{i}\left(\mathbf{r}_{i}\right) \frac{1}{2} \nabla^{2} n_{j}\left(\mathbf{r}_{i}\right):\left(\mathbf{r}_{i j} \otimes \mathbf{r}_{i j}\right) \mathrm{d} \mathbf{r}_{i j} .
$$

After some algebra, using the van der Waals and Rayleigh expression (5.6) for the capillarity coefficient, the resulting extra volumetric energy density $\mathcal{E}^{\text {ex }}$ reads

$$
\mathcal{E}^{\mathrm{ex}}=-\sum_{i, j \in \mathfrak{S}} \frac{1}{2} \varkappa_{i j} n_{i} \Delta n_{j}
$$

With the aim of simplifying (5.8), we may use the identity

$$
-\sum_{i, j \in \mathfrak{S}} \frac{1}{2} \varkappa_{i j} n_{i} \Delta n_{j}=\sum_{i, j \in \mathfrak{S}} \frac{1}{2} \varkappa_{i j} \nabla n_{i} \cdot \nabla n_{j}-\sum_{i, j \in \mathfrak{S}} \frac{1}{2} \nabla \cdot\left(\varkappa_{i j} n_{i} \nabla n_{j}\right),
$$

in order to recover the energy density in agreement with (2.7)

$$
\overline{\mathcal{E}}^{\mathrm{ex}}=\sum_{i, j \in \mathfrak{S}} \frac{1}{2} \varkappa_{i j} \nabla n_{i} \cdot \nabla n_{j},
$$

provided we take into account the residual terms $-\sum_{i, j \in \mathfrak{S}} \frac{1}{2} \boldsymbol{\nabla} \cdot\left(\varkappa_{i j} n_{i} \boldsymbol{\nabla} n_{j}\right)$. By changing the energy density from $\mathcal{E}^{\mathrm{ex}}$ into $\overline{\mathcal{E}}^{\mathrm{ex}}$ we indeed have to add an extra corrector term to the energy equation in the form

$$
\Xi=-\sum_{i, j \in \mathfrak{S}} \frac{1}{2}\left(\partial_{t} \boldsymbol{\nabla} \cdot\left(\varkappa_{i j} n_{i} \nabla n_{j}\right)+\nabla \cdot\left(\boldsymbol{\nabla} \cdot\left(\varkappa_{i j} n_{i} \nabla n_{j}\right) \boldsymbol{v}\right)\right) .
$$

We may rewrite this corrector as the divergence of a corrector flux $\mathfrak{q}_{0}$ by using the zeroth order species mass conservation equations $\partial_{t} n_{i}=-\boldsymbol{\nabla} \cdot\left(n_{i} \boldsymbol{v}\right), i \in \mathfrak{S}$, keeping in mind that the capillarity coefficients $\varkappa_{i j}$ are taken to be constant. After some algebra, the corrector term $\Xi$ is obtained in the form $\Xi=\nabla \cdot \mathfrak{q}_{0}$ where

$$
\begin{aligned}
\mathfrak{q}_{0}= & \sum_{i, j \in \mathfrak{S}} \varkappa_{i j} n_{i} \boldsymbol{\nabla} \cdot \boldsymbol{v} \boldsymbol{\nabla} n_{j}+\sum_{i, j \in \mathfrak{S}} \frac{1}{2} \varkappa_{i j} n_{i} n_{j} \boldsymbol{\nabla}(\boldsymbol{\nabla} \cdot \boldsymbol{v})+\sum_{i, j \in \mathfrak{S}} \frac{1}{2} \varkappa_{i j} n_{i}(\boldsymbol{\nabla} \boldsymbol{v})^{t} \cdot \boldsymbol{\nabla} n_{j} \\
& +\sum_{i, j \in \mathfrak{S}} \frac{1}{2} \varkappa_{i j}\left(\boldsymbol{\nabla} n_{i} \otimes \boldsymbol{\nabla} n_{j}+n_{i} \boldsymbol{\nabla}^{2} n_{j}-\boldsymbol{\nabla} n_{i} \cdot \boldsymbol{\nabla} n_{j} \boldsymbol{I}-n_{i} \Delta n_{j} \boldsymbol{I}\right) \cdot \boldsymbol{v} .
\end{aligned}
$$

The change of energy density from $\mathcal{E}^{\mathrm{ex}}=-\sum_{i, j \in \mathfrak{S}} \frac{1}{2} \varkappa_{i j} n_{i} \Delta n_{j}$ into the gradient expression $\overline{\mathcal{E}}^{\text {ex }}=\sum_{i, j \in \mathfrak{S}} \frac{1}{2} \varkappa_{i j} \nabla n_{i} \cdot \nabla n_{j}$ has been addressed when investigating diffuse interface single species fluids [15]. Aside from this derivation [15], it has been advocated in previous work that $\mathcal{E}^{\text {ex }}$ may be transformed into $\overline{\mathcal{E}}^{\text {ex }}$ by integrating by parts over 'a large 
volume' with boundary conditions insuring a null contribution of boundary terms. This is unsatisfactory since an energy density must be a local quantity, not defined over 'a large volume' or else under the nonlocal influence of a far boundary with unclear definition and unclear boundary conditions. Following the derivation of single species diffuse interface fluid models [15], by properly taking into account the correction heat flux $\mathfrak{q}_{0}$, we clarify the local change of Cahn-Hilliard energy density from $\mathcal{E}^{\mathrm{ex}}$ to $\overline{\mathcal{E}}^{\mathrm{ex}}$ for mixtures of fluids. Finally, since the capillarity coefficients are taken to be constant, we must have from thermodynamics that $\bar{p}^{\mathrm{ex}}=-\overline{\mathcal{E}}^{\mathrm{ex}}$ so that the pressure is in the form $p=p^{\mathrm{u}}-\sum_{i, j \in \mathfrak{S}} \frac{1}{2} \varkappa_{i j} \boldsymbol{\nabla} n_{i} \cdot \nabla n_{j}$ and similarly we must have $\bar{g}_{i}=\bar{g}_{i}^{\mathrm{u}}$.

\subsection{A generalized Dunn and Serrin heat flux}

In order to evaluate the extra heat flux $\mathcal{Q}^{\text {ex }}$ arising from Taylor expansions of the zeroth pair distribution functions $f_{i j}^{(0), \text { sy }}$ in $\mathcal{Q}_{1}^{\mathrm{P}}$ and $\mathcal{Q}_{2}^{\mathrm{P}}$ and using (5.1), we need to evaluate the gradients of $f_{i}^{(0)}$. After some algebra we note that

$$
\frac{\boldsymbol{\nabla} f_{i}^{(0)}}{f_{i}^{(0)}}=\frac{m_{i}}{k_{\mathrm{B}} T}\left(\boldsymbol{c}_{i}-\boldsymbol{v}\right) \cdot \boldsymbol{\nabla} \boldsymbol{v}+\frac{\boldsymbol{\nabla} n_{i}}{n_{i}}-\left(\frac{3}{2}-\frac{m_{i}\left|\boldsymbol{c}_{i}-\boldsymbol{v}\right|^{2}}{2 k_{\mathrm{B}} T}\right) \frac{\boldsymbol{\nabla} T}{T},
$$

where all gradients are of course evaluated at $\mathbf{r}_{i}$ and similarly that

$$
\begin{aligned}
\frac{\boldsymbol{\nabla}^{2} f_{i}^{(0)}}{f_{i}^{(0)}} & =\frac{\boldsymbol{\nabla} f_{i}^{(0)} \otimes \boldsymbol{\nabla} f_{i}^{(0)}}{\left(f_{i}^{(0)}\right)^{2}}-\frac{m_{i}}{k_{\mathrm{B}} T} \boldsymbol{\nabla} \boldsymbol{v} \cdot \boldsymbol{\nabla} \boldsymbol{v}+\frac{m_{i}}{k_{\mathrm{B}} T}\left(\boldsymbol{c}_{i}-\boldsymbol{v}\right) \cdot \boldsymbol{\nabla}^{2} \boldsymbol{v} \\
& -\frac{m_{i}}{k_{\mathrm{B}} T^{2}}\left(\left(\boldsymbol{c}_{i}-\boldsymbol{v}\right) \cdot \boldsymbol{\nabla} \boldsymbol{v}\right) \otimes \boldsymbol{\nabla} T-\frac{m_{i}}{k_{\mathrm{B}} T^{2}} \boldsymbol{\nabla} \otimes\left(\left(\boldsymbol{c}_{i}-\boldsymbol{v}\right) \cdot \boldsymbol{\nabla} \boldsymbol{v}\right)+\frac{\boldsymbol{\nabla}^{2} n_{i}}{n_{i}} \\
& -\frac{\boldsymbol{\nabla} n_{i} \otimes \boldsymbol{\nabla} n_{i}}{n_{i}^{2}}-\left(\frac{3}{2}-\frac{m_{i}\left|\boldsymbol{c}_{i}-\boldsymbol{v}\right|^{2}}{2 k_{\mathrm{B}} T}\right) \frac{\boldsymbol{\nabla}^{2} T}{T}+\left(\frac{3}{2}-\frac{m_{i}\left|\boldsymbol{c}_{i}-\boldsymbol{v}\right|^{2}}{k_{\mathrm{B}} T}\right) \frac{\boldsymbol{\nabla} T \otimes \boldsymbol{\nabla} T}{T^{2}}
\end{aligned}
$$

With $\mathcal{Q}_{1}^{\text {P }}$ given by (3.19), we first need to select the integrands obtained from a Taylor expansion of $f_{i}^{(0)}\left(\mathbf{r}_{i}, \boldsymbol{c}_{i}, t\right) f_{j}^{(0)}\left(\mathbf{r}_{j}, \boldsymbol{c}_{j}, t\right)$ that involve the peculiar velocity of the first particle $\boldsymbol{c}_{i}-\boldsymbol{v}$ as well as two spatial derivatives and that are even in $\mathbf{r}_{i j}$. It appears, however, that there are not such terms in the Taylor expansion of $f_{i}^{(0)}\left(\mathbf{r}_{i}, \boldsymbol{c}_{i}, t\right) f_{j}^{(0)}\left(\mathbf{r}_{j}, \boldsymbol{c}_{j}, t\right)$ so that there is no extra capillary heat flux arising from $\mathcal{Q}_{1}^{\mathrm{P}}$.

On the other hand, with $\mathcal{Q}_{2}^{\text {P }}$ given by (3.20), from the isotropy of space and velocity space, we need to select the integrands in the Taylor expansion of

$$
f_{i}^{(0)}\left(\mathbf{r}_{i}-(1-\alpha) \mathbf{r}_{i j}, \boldsymbol{c}_{i}, t\right) f_{j}^{(0)}\left(\mathbf{r}_{i}+\alpha \mathbf{r}_{i j}, \boldsymbol{c}_{j}, t\right) g_{i j}\left(r_{i j}\right),
$$

that involve two derivatives, are even with respect to $\mathbf{r}_{i j}$, and odd with respect to either $\boldsymbol{c}_{i}-\boldsymbol{v}$ or $\boldsymbol{c}_{j}-\boldsymbol{v}$. We first focus of integrands having such properties arising from the cross products of two first order derivatives of $f_{i}^{(0)}$ and $f_{j}^{(0)}$ that are

$$
-\alpha(1-\alpha) \frac{m_{i}}{k_{\mathrm{B}} T}\left(\boldsymbol{c}_{i}-\boldsymbol{v}\right) \cdot \boldsymbol{\nabla} \boldsymbol{v} \cdot \mathbf{r}_{i j} \frac{\boldsymbol{\nabla} n_{j} \cdot \mathbf{r}_{i j}}{n_{j}}-\alpha(1-\alpha) \frac{m_{j}}{k_{\mathrm{B}} T}\left(\boldsymbol{c}_{j}-\boldsymbol{v}\right) \cdot \boldsymbol{\nabla} \boldsymbol{v} \cdot \mathbf{r}_{i j} \frac{\boldsymbol{\nabla} n_{i} \cdot \mathbf{r}_{i j}}{n_{i}},
$$

as well as

$$
\begin{aligned}
\alpha(1-\alpha) & \frac{m_{i}}{k_{\mathrm{B}} T}\left(\boldsymbol{c}_{i}-\boldsymbol{v}\right) \cdot \boldsymbol{\nabla} \boldsymbol{v} \cdot \mathbf{r}_{i j}\left(\frac{3}{2}-\frac{m_{j}\left|\boldsymbol{c}_{j}-\boldsymbol{v}\right|^{2}}{2 k_{\mathrm{B}} T}\right) \frac{\boldsymbol{\nabla} T \cdot \mathbf{r}_{i j}}{T} \\
& +\alpha(1-\alpha) \frac{m_{j}}{k_{\mathrm{B}} T}\left(\boldsymbol{c}_{j}-\boldsymbol{v}\right) \cdot \boldsymbol{\nabla} \boldsymbol{v} \cdot \mathbf{r}_{i j}\left(\frac{3}{2}-\frac{m_{i}\left|\boldsymbol{c}_{i}-\boldsymbol{v}\right|^{2}}{2 k_{\mathrm{B}} T}\right) \frac{\boldsymbol{\nabla} T \cdot \mathbf{r}_{i j}}{T} .
\end{aligned}
$$


The last integrands yield vanishing contributions for each species pair since

$$
\int\left(\frac{3}{2}-\frac{m_{i}\left|\boldsymbol{c}_{i}-\boldsymbol{v}\right|^{2}}{2 k_{\mathrm{B}} T}\right) f_{i}^{(0)} \mathrm{d} \boldsymbol{c}_{i}=0, \quad i \in \mathfrak{S} .
$$

Using then $\int_{0}^{1} \alpha(1-\alpha) \mathrm{d} \alpha=\frac{1}{6}$ as well as the isotropy of the velocity space and elementary symmetries, we next obtain from the first integrands a contribution $\mathfrak{q}_{1}$ for the heat flux in the form

$$
\begin{aligned}
\mathfrak{q}_{1}=\sum_{i, j \in \mathfrak{S}} \frac{1}{12} \int \frac{\varphi_{i j}^{\prime}\left(r_{i j}\right)}{r_{i j}} \mathbf{r}_{i j} \mathbf{r}_{i j} \cdot\left(\boldsymbol{c}_{i}-\boldsymbol{v}\right) \frac{m_{i}}{k_{\mathrm{B}} T}\left(\boldsymbol{c}_{i}-\boldsymbol{v}\right) \cdot \boldsymbol{\nabla} \boldsymbol{v} \cdot \mathbf{r}_{i j} \frac{\boldsymbol{\nabla} n_{j} \cdot \mathbf{r}_{i j}}{n_{j}} \\
\times f_{i}^{(0)}\left(\mathbf{r}_{i}, \boldsymbol{c}_{i}\right) f_{i}^{(0)}\left(\mathbf{r}_{i}, \boldsymbol{c}_{j}\right) g_{i j}\left(r_{i j}\right) \mathrm{d} \boldsymbol{c}_{i} \mathrm{~d} \mathbf{r}_{i j} \mathrm{~d} \boldsymbol{c}_{j} .
\end{aligned}
$$

The integrations over $\boldsymbol{c}_{j}$ are trivial and yield factors $n_{j}$, the integrations over $\boldsymbol{c}_{i}$ are performed using the reduced velocities $\left(\boldsymbol{c}_{i}-\boldsymbol{v}\right)\left(m_{i} / 2 k_{\mathrm{B}} T\right)^{1 / 2}$ and (F.1) from Appendix F, and this yields

$$
\mathfrak{q}_{1}=\sum_{i, j \in \mathfrak{S}} \frac{1}{12} n_{i} \int \frac{\varphi_{i j}^{\prime}\left(r_{i j}\right)}{r_{i j}} \mathbf{r}_{i j} \otimes \mathbf{r}_{i j} \nabla \boldsymbol{v}:\left(\mathbf{r}_{i j} \otimes \mathbf{r}_{i j}\right) g_{i j}\left(r_{i j}\right) \mathrm{d} \mathbf{r}_{i j} \cdot \nabla n_{j}
$$

Using the differential identity (F.3) we then obtain that $\mathfrak{q}_{1}$ is in the form

$$
\mathfrak{q}_{1}=\sum_{i, j \in \mathfrak{S}} \frac{\varkappa_{i j}}{6} n_{i}\left(\boldsymbol{\nabla} \boldsymbol{v}+(\boldsymbol{\nabla} \boldsymbol{v})^{t}+\boldsymbol{\nabla} \cdot \boldsymbol{v} \boldsymbol{I}\right) \cdot \boldsymbol{\nabla} n_{j}
$$

with the cohesion coefficients $\varkappa_{i j}, i, j \in \mathfrak{S}$, given by (5.2).

We now focus on contributions arising from the second order derivatives $\nabla^{2} f_{i}^{(0)}$ or $\nabla^{2} f_{j}^{(0)}$ in the Taylor expansion of $f_{i}^{(0)}\left(\mathbf{r}_{i}-(1-\alpha) \mathbf{r}_{i j}, \boldsymbol{c}_{i}, t\right) f_{j}^{(0)}\left(\mathbf{r}_{i}+\alpha \mathbf{r}_{i j}, \boldsymbol{c}_{j}, t\right)$. The relevant integrands are those even with respect to $\mathbf{r}_{i j}$ and odd with respect to either $\boldsymbol{c}_{i}-\boldsymbol{v}$ or $\boldsymbol{c}_{j}-\boldsymbol{v}$. These integrands are first

$$
\begin{aligned}
& \alpha^{2} \frac{2 m_{i}}{k_{\mathrm{B}} T}\left(\boldsymbol{c}_{i}-\boldsymbol{v}\right) \cdot \boldsymbol{\nabla} \boldsymbol{v} \cdot \mathbf{r}_{i j} \frac{\boldsymbol{\nabla} n_{j} \cdot \mathbf{r}_{i j}}{n_{j}}+(1-\alpha)^{2} \frac{2 m_{j}}{k_{\mathrm{B}} T}\left(\boldsymbol{c}_{j}-\boldsymbol{v}\right) \cdot \boldsymbol{\nabla} \boldsymbol{v} \cdot \mathbf{r}_{i j} \frac{\boldsymbol{\nabla} n_{i} \cdot \mathbf{r}_{i j}}{n_{i}}, \\
& \quad+\alpha^{2} \frac{m_{i}}{k_{\mathrm{B}} T}\left(\boldsymbol{c}_{i}-\boldsymbol{v}\right) \cdot \nabla^{2} \boldsymbol{v}:\left(\mathbf{r}_{i j} \otimes \mathbf{r}_{i j}\right)+(1-\alpha)^{2} \frac{m_{j}}{k_{\mathrm{B}} T}\left(\boldsymbol{c}_{j}-\boldsymbol{v}\right) \cdot \nabla^{2} \boldsymbol{v}:\left(\mathbf{r}_{i j} \otimes \mathbf{r}_{i j}\right) .
\end{aligned}
$$

The two first integrands yields a contribution $\mathfrak{q}_{2}$ similar to $\mathfrak{q}_{1}$ obtained in (5.14) but with the coefficient $-\frac{1}{3}$ instead on $\frac{1}{6}$.

$$
\mathfrak{q}_{2}=-\sum_{i, j \in \mathfrak{S}} \frac{\varkappa_{i j}}{3} n_{i}\left(\boldsymbol{\nabla} \boldsymbol{v}+(\boldsymbol{\nabla} \boldsymbol{v})^{t}+\boldsymbol{\nabla} \cdot \boldsymbol{v} \boldsymbol{I}\right) \cdot \boldsymbol{\nabla} n_{j} .
$$

Using the isotropy of space and elementary symmetries, the integrands associated with $\nabla^{2} v$ further yield the contributions

$$
\begin{aligned}
\mathbf{q}_{3}=-\sum_{i, j \in \mathfrak{S}} \frac{1}{12} \int \frac{\varphi_{i j}^{\prime}\left(r_{i j}\right)}{r_{i j}} \mathbf{r}_{i j} \mathbf{r}_{i j} & \left(\boldsymbol{c}_{i}-\boldsymbol{v}\right) \frac{m_{i}}{k_{\mathrm{B}} T}\left(\boldsymbol{c}_{i}-\boldsymbol{v}\right) \cdot \nabla^{2} \boldsymbol{v}:\left(\mathbf{r}_{i j} \otimes \mathbf{r}_{i j}\right) \\
& \times f_{i}^{(0)}\left(\mathbf{r}_{i}, \boldsymbol{c}_{i}\right) f_{i}^{(0)}\left(\mathbf{r}_{i}, \boldsymbol{c}_{j}\right) g_{i j}\left(r_{i j}\right) \mathrm{d} \boldsymbol{c}_{i} \mathrm{~d} \mathbf{r}_{i j} \mathrm{~d} \boldsymbol{c}_{j} .
\end{aligned}
$$


The integrations over $\boldsymbol{c}_{j}$ yield the factors $n_{j}$, the integrations over $\boldsymbol{c}_{i}$ are obtained with (F.1) from Appendix F so that

$$
\mathfrak{q}_{3}=-\sum_{i, j \in \mathfrak{S}} \frac{1}{12} n_{i} n_{j} \int \frac{\varphi_{i j}^{\prime}\left(r_{i j}\right)}{r_{i j}} \mathbf{r}_{i j} \mathbf{r}_{i j} \cdot \nabla^{2} \boldsymbol{v}:\left(\mathbf{r}_{i j} \otimes \mathbf{r}_{i j}\right) g_{i j}\left(r_{i j}\right) \mathrm{d} \mathbf{r}_{i j} .
$$

We may then use the identity (F.6) with $\nabla^{2} \boldsymbol{v}:\left(\mathbf{r}_{i j} \otimes \mathbf{r}_{i j} \otimes \mathbf{r}_{i j}\right)=\mathbf{r}_{i j} \cdot \nabla^{2} \boldsymbol{v}:\left(\mathbf{r}_{i j} \otimes \mathbf{r}_{i j}\right)$ to deduce that

$$
\mathfrak{q}_{3}=-\sum_{i, j \in \mathfrak{S}} \frac{1}{6} n_{i} n_{j} \varkappa_{i j}(\Delta \boldsymbol{v}+2 \boldsymbol{\nabla}(\boldsymbol{\nabla} \cdot \boldsymbol{v})) .
$$

Finally, there are also integrands associated with temperature gradients in the form

$$
-\frac{2 m_{i}}{k_{\mathrm{B}} T}\left(\boldsymbol{c}_{i}-\boldsymbol{v}\right) \cdot \boldsymbol{\nabla} \boldsymbol{v} \cdot \mathbf{r}_{i j}\left(\frac{3}{2}-\frac{m_{i}\left|\boldsymbol{c}_{i}-\boldsymbol{v}\right|^{2}}{2 k_{\mathrm{B}} T}\right) \frac{\boldsymbol{\nabla} T \cdot \mathbf{r}_{i j}}{T}-\frac{2 m_{i}}{k_{\mathrm{B}} T}\left(\boldsymbol{c}_{i}-\boldsymbol{v}\right) \cdot \boldsymbol{\nabla} \boldsymbol{v} \cdot \mathbf{r}_{i j} \frac{\boldsymbol{\nabla} T \cdot \mathbf{r}_{i j}}{T},
$$

with a similar expression in terms of $\left(\boldsymbol{c}_{j}-\boldsymbol{v}\right)$ but all these terms yield vanishing contributions since

$$
\int\left|\boldsymbol{c}_{i}-\boldsymbol{v}\right|^{2}\left(\frac{5}{2}-\frac{m_{i}\left|\boldsymbol{c}_{i}-\boldsymbol{v}\right|^{2}}{2 k_{\mathrm{B}} T}\right) f_{i}^{(0)} \mathrm{d} \boldsymbol{c}_{i}=0
$$

Collecting previous results, we have obtained an extra total energy flux $\mathcal{Q}^{\text {ex }}+\mathcal{P}^{\text {ex }} \cdot \boldsymbol{v}$ in the form

$$
\mathcal{Q}^{\mathrm{ex}}+\mathcal{P}^{\mathrm{ex}} \cdot \boldsymbol{v}=\mathfrak{q}_{0}+\mathfrak{q}_{1}+\mathfrak{q}_{2}+\mathfrak{q}_{3}+\mathcal{P}^{\mathrm{ex}} \cdot \boldsymbol{v},
$$

where $\mathfrak{q}_{0}$ arise from the change of energy density. The extra total energy flux $\mathcal{Q}^{\text {ex }}+\mathcal{P}^{\text {ex }} \cdot \boldsymbol{v}$ thus first reads

$$
\begin{aligned}
\mathcal{Q}^{\mathrm{ex}}+ & \mathcal{P}^{\mathrm{ex}} \cdot \boldsymbol{v}=\sum_{i, j \in \mathfrak{S}} \varkappa_{i j} n_{i} \boldsymbol{\nabla} \cdot \boldsymbol{v} \boldsymbol{\nabla} n_{j}+\sum_{i, j \in \mathfrak{S}} \frac{1}{2} \varkappa_{i j} n_{i} n_{j} \boldsymbol{\nabla}(\boldsymbol{\nabla} \cdot \boldsymbol{v})+\sum_{i, j \in \mathfrak{S}} \frac{1}{2} \varkappa_{i j} n_{i}(\boldsymbol{\nabla} \boldsymbol{v})^{t} \cdot \boldsymbol{\nabla} n_{j} \\
& +\sum_{i, j \in \mathfrak{S}} \frac{1}{2} \varkappa_{i j}\left(\boldsymbol{\nabla} n_{i} \otimes \boldsymbol{\nabla} n_{j}+n_{i} \boldsymbol{\nabla}^{2} n_{j}-\nabla n_{i} \cdot \boldsymbol{\nabla} n_{j} \boldsymbol{I}-n_{i} \Delta n_{j}\right) \cdot \boldsymbol{v} \\
& -\sum_{i, j \in \mathfrak{S}} \frac{\varkappa_{i j}}{6} n_{i}\left(\boldsymbol{\nabla} \boldsymbol{v}+(\boldsymbol{\nabla} \boldsymbol{v})^{t}+\boldsymbol{\nabla} \cdot \boldsymbol{v} \boldsymbol{I}\right) \cdot \boldsymbol{\nabla} n_{j}-\sum_{i, j \in \mathfrak{S}} \frac{\varkappa_{i j}}{6} n_{i} n_{j}(\Delta \boldsymbol{v}+2 \boldsymbol{\nabla}(\boldsymbol{\nabla} \cdot \boldsymbol{v})) \\
& +\sum_{i, j \in \mathfrak{S}} \frac{\varkappa_{i j}}{6}\left(2 \boldsymbol{\nabla} n_{i} \otimes \boldsymbol{\nabla} n_{j}+\nabla n_{i} \cdot \boldsymbol{\nabla} n_{j} \boldsymbol{I}-4 n_{i} \boldsymbol{\nabla}^{2} n_{j}-2 n_{i} \Delta n_{j} \boldsymbol{I}\right) \cdot \boldsymbol{v} .
\end{aligned}
$$

In order to simplify this expression we seek an equivalent expression $\overline{\mathcal{Q}}^{\text {ex }}$ of $\mathcal{Q}^{\text {ex }}$, that is, we seek a flux $\overline{\mathcal{Q}}^{\text {ex }}$ such that

$$
\boldsymbol{\nabla} \cdot\left(\mathcal{Q}^{\mathrm{ex}}+\mathcal{P}^{\mathrm{ex}} \cdot \boldsymbol{v}\right)=\boldsymbol{\nabla} \cdot\left(\overline{\mathcal{Q}}^{\mathrm{ex}}+\overline{\mathcal{P}}^{\mathrm{ex}} \cdot \boldsymbol{v}\right),
$$

allowing the simultaneous use of the simplified heat flux $\overline{\mathcal{Q}}^{\text {ex }}$ and the simplified pressure tensor $\overline{\mathcal{P}}^{\text {ex }}$. In order to simplify the divergence of $\mathcal{Q}^{\text {ex }}+\mathcal{P}^{\text {ex }} \cdot \boldsymbol{v}$, the first terms $\varkappa_{i j} n_{i} \boldsymbol{\nabla} \cdot \boldsymbol{v} \boldsymbol{\nabla} n_{j}$ are left unchanged since they corresponds to a generalized Dunn and Serrin heat flux, all terms proportional to $\boldsymbol{v}$ are regrouped, and the differential identity (F.8) is used to transform the divergence of all the terms $-\frac{1}{6} \varkappa_{i j} n_{i} \boldsymbol{\nabla} \cdot \boldsymbol{v} \boldsymbol{\nabla} n_{j}$ in the third line that originate from $\mathfrak{q}_{1}+\mathfrak{q}_{2}$. After some lengthy algebra this yields the expression

$$
\begin{aligned}
\boldsymbol{\nabla} \cdot\left(\mathcal{Q}^{\mathrm{ex}}+\mathcal{P}^{\mathrm{ex}} \cdot \boldsymbol{v}\right) & =\sum_{i, j \in \mathfrak{S}} \boldsymbol{\nabla} \cdot\left(\varkappa_{i j} n_{i} \boldsymbol{\nabla} \cdot \boldsymbol{v} \boldsymbol{\nabla} n_{j}-\frac{1}{3} \varkappa_{i j} n_{i} \boldsymbol{\nabla} \boldsymbol{v} \cdot \boldsymbol{\nabla} n_{j}\right. \\
& +\frac{1}{3} \varkappa_{i j} n_{i}(\boldsymbol{\nabla} \boldsymbol{v})^{t} \cdot \boldsymbol{\nabla} n_{j}-\frac{1}{6} \varkappa_{i j} n_{i} n_{j} \Delta \boldsymbol{v}+\frac{1}{6} \varkappa_{i j} n_{i} n_{j} \boldsymbol{\nabla}(\boldsymbol{\nabla} \cdot \boldsymbol{v}) \\
& \left.+\varkappa_{i j}\left(\boldsymbol{\nabla} n_{i} \otimes \boldsymbol{\nabla} n_{j}-\frac{1}{2} \boldsymbol{\nabla} n_{i} \cdot \boldsymbol{\nabla} n_{j} \boldsymbol{I}-n_{i} \Delta n_{j} \boldsymbol{I}\right) \cdot \boldsymbol{v}\right) .
\end{aligned}
$$


Using then the differential identity (F.9), the divergence of the second to fifth terms (four terms) on the right hand side vanishes for each species pair $(i, j)$, and we obtain

$$
\begin{gathered}
\boldsymbol{\nabla} \cdot\left(\mathcal{Q}^{\mathrm{ex}}+\mathcal{P}^{\mathrm{ex}} \cdot \boldsymbol{v}\right)=\sum_{i, j \in \mathfrak{S}} \boldsymbol{\nabla} \cdot\left(\varkappa_{i j} n_{i} \boldsymbol{\nabla} \cdot \boldsymbol{v} \nabla n_{j}+\varkappa_{i j}\left(\boldsymbol{\nabla} n_{i} \otimes \nabla n_{j}\right.\right. \\
\left.\left.-\frac{1}{2} \boldsymbol{\nabla} n_{i} \cdot n_{j} \boldsymbol{I}-n_{i} \Delta n_{j} \boldsymbol{I}\right) \cdot \boldsymbol{v}\right)
\end{gathered}
$$

so that the equivalent heat flux $\overline{\mathcal{Q}}^{\mathrm{ex}}$ is given by

$$
\overline{\mathcal{Q}}^{\mathrm{ex}}=\sum_{i, j \in \mathfrak{S}} \varkappa_{i j} n_{i} \boldsymbol{\nabla} \cdot \boldsymbol{v} \nabla n_{j} .
$$

Using the mass based capillarity coefficients $\bar{\varkappa}_{i j}=\varkappa_{i j} / m_{i} m_{j}$, we obtain that the extra flux $\overline{\mathcal{Q}}^{\text {ex }}$ coincides with the generalized Dunn and Serrin heat flux $\overline{\mathcal{Q}}^{\text {ex }}=\mathcal{Q}^{\text {DS }}$ as derived from rational thermodynamics. A key point in the simplification of the zeroth order capillary heat flux was notably the use of the modified density energy $\overline{\mathcal{E}}^{\text {ex }}$ in order to compensate complex corrections arising from $\mathcal{Q}_{2}^{\text {P }}$ by similar terms arising from $\mathfrak{q}_{0}$.

In summary, the generalized Korteweg tensor (2.18) has been recovered as well as the generalized Dunn and Serrin heat flux (2.19) at zeroth order. We have also established that the energy density is in the form $\mathcal{E}=\mathcal{E}^{\mathrm{u}}+\sum_{i, j \in \mathfrak{S}} \frac{1}{2} \varkappa_{i j} \nabla n_{i} \cdot \nabla n_{j}$. The full capillary equations have thus been recovered at zeroth order and we now have to investigate the first order regime, that is, we have to recover force terms $\boldsymbol{X}_{i}$ that include the CahnHilliard extra forces $\boldsymbol{X}_{i}^{\text {ch }}$, for $i \in \mathfrak{S}$, as well as the extra capillary-diffusive heat flux $\mathcal{Q}^{\mathrm{CD}}$.

\section{Cahn-Hilliard fluid equations}

We derive in this section the Cahn-Hilliard fluid equations at first order of the ChapmanEnskog method. To this aim, we investigate higher order derivative terms in the linearized equations and in the governing equations at first order of Enskog expansion. This is done by using the out of equilibrium symmetrized pair distribution functions $f_{i j}^{\text {sy }}$ as well as higher order Taylor expansions. The resulting equations then coincide with the model derived from rational thermodynamics. We also address the use of the pair distribution functions $f_{i j}^{\text {Bo }}$ suggested by Bogoliubov theory and establish that the deviations arising from the differences $f_{i j}^{\text {Bo }}-f_{i j}^{\text {sy }}$ may be seen as perturbations in some regime.

\subsection{Cahn-Hilliard diffusion driving forces}

The generalized Boltzmann equations (4.1) and the use of higher order Taylor expansions of pair distribution functions have led to the Euler/van der Waals equations at zeroth order. At first order of Enskog expansion (4.2), we next need to evaluate the linearized equations governing the perturbed distribution functions $\phi^{(1)}=\left(\phi_{i}^{(1)}\right)_{i \in \mathfrak{S}}$. The linearized equations have been obtained in the form (4.18) with the linearized operator (4.19) and right hand sides (4.20)(4.21). The right hand sides (4.21) have only been evaluated using first order Taylor expansions of pair distribution functions in Section 4 or Appendix D. We must therefore reevaluate these right hand sides using higher order Taylor expansions of zeroth order pair distribution functions. We denote with the superscript ${ }^{\mathrm{u}}$ the bulk phase thermodynamic properties obtained in Section 4 that do not involve gradients. The 
higher order derivative terms are again evaluated by using the symmetrized zeroth order pair distribution functions $f_{i j}^{(0) \text {,sy }}, i, j \in \mathfrak{S}$.

From the general expression of the right hand sides (4.20), we need to evaluate the extra terms arising from the material derivatives $\partial_{t} \log f_{i}^{(0)}+\boldsymbol{v} \cdot \boldsymbol{\nabla} \log f_{i}^{(0)}$ as well as those arising from the potential parts $\mathcal{J}_{i}^{(1)}\left(f^{(0)}\right) / f_{i}^{(0)}$. From Euler equations, derived by using the pair distribution functions $f_{i j}^{(0) \text {,sy }}$, there are new terms arising in the material derivatives $\partial_{t} \log f^{(0)}+\boldsymbol{v} \cdot \nabla \log f^{(0)}$. More specifically, using (5.4), (5.8), (5.17), we obtain after some calculus the multicomponent Euler equations with diffuse interface terms in the form

$$
\begin{aligned}
& \partial_{t} n_{i}+\boldsymbol{v} \cdot n_{i}=-n_{i} \boldsymbol{\nabla} \cdot \boldsymbol{v}, \quad i \in \mathfrak{S}, \\
& \partial_{t} \boldsymbol{v}+\boldsymbol{v} \cdot \boldsymbol{v}=-\frac{\boldsymbol{\nabla} p^{\mathrm{u}}}{\rho}+\sum_{i, j \in \mathfrak{S}} \frac{\varkappa_{i j} n_{i}}{\rho} \nabla \Delta n_{j}, \\
& \partial_{t} T+\boldsymbol{v} \cdot T=\frac{1}{\partial_{T} \mathcal{E}^{\mathrm{u}}}\left(\sum_{l \in \mathfrak{S}} n_{l} \partial_{n_{l}} \mathcal{E}^{\mathrm{u}}-\mathcal{E}^{\mathrm{u}}-p^{\mathrm{u}}\right) \boldsymbol{\nabla} \cdot \boldsymbol{v},
\end{aligned}
$$

instead of the standard Euler equations (D.2)-(D.4). In particular, there is a cancellation of diffusive interface terms in the energy equation at zeroth order $\partial_{t} \mathcal{E}^{\mathrm{u}}+\boldsymbol{\nabla} \cdot\left(\boldsymbol{v} \mathcal{E}^{\mathrm{u}}\right)+$ $p^{\mathrm{u}} \boldsymbol{\nabla} \cdot \boldsymbol{v}=0$ as well as in the corresponding temperature equation (6.3). There is, however, a diffuse interface term in the momentum conservation equation (6.2) involving the capillary forces.

In order to evaluate the material derivative of $\log f_{i}^{(0)}$, we may use the expression of Maxwellian distributions as well as (6.1)-(6.3) and is obtained that

$$
-\left(\partial_{t} \log f_{i}^{(0)}+\boldsymbol{v} \cdot \nabla \log f_{i}^{(0)}\right)=\Psi_{i}^{\mathrm{K}, 1}+\Psi_{i}^{\mathrm{K}, 3},
$$

where $\Psi_{i}^{\mathrm{K}, 1}$ has already been evaluated in Section 4 and Appendix D and where $\Psi_{i}^{\mathrm{K}, 3}$ is obtained from (6.1)-(6.3) in the form

$$
\Psi_{i}^{\mathrm{K}, 3}=-\frac{m_{i}\left(\boldsymbol{c}_{i}-\boldsymbol{v}\right)}{\rho k_{\mathrm{B}} T} \cdot \sum_{k, l \in \mathfrak{S}} \varkappa_{k l} n_{k} \boldsymbol{\nabla} \Delta n_{l},
$$

with the third order derivative terms solely arising from the force term in (6.2).

We next need to investigate the higher order terms arising from the potential parts $\mathcal{J}_{i}^{(1)}\left(f^{(0)}\right) / f_{i}^{(0)}$ of the right hand sides $\psi_{i}^{(1)}, i \in \mathfrak{S}$. Using the general definition of the perturbed operator (3.34), we may write

$$
\frac{1}{f_{i}^{(0)}} \mathcal{J}_{i}^{(1)}\left(f^{(0)}\right)=\Psi_{i}^{\mathrm{P}, 1}+\Psi_{i}^{\mathrm{P}, 3}
$$

where the first order terms $\Psi_{i}^{\mathrm{P}, 1}$ have already been investigated in Section 4 and Appendix D whereas the contributions $\Psi_{i}^{\mathrm{P}, 3}$ arise from third order terms in Taylor expansion of pair distribution functions. All quadratic terms are indeed even in $\mathbf{r}_{i j}$ or and $\mathbf{r}_{i j}^{\prime}$ and yield zero contributions in the expansion of $\mathcal{J}_{i}^{(1)}\left(f^{(0)}\right)$.

In order to evaluate the third order derivative contributions $\Psi_{i}^{\mathrm{P}, 3}$ arising from the perturbed source term $\mathcal{J}_{i}^{(1)}\left(f^{(0)}\right)$, for consistency with the Euler equations, and for the sake of simplicity, we again use the symmetrized pair distribution functions $f_{i j}^{(0), \text { sy }}$. In 
other words, keeping in mind the zeroth order identity $(4.5)$, the corrector terms $f_{i j}^{(0), \text { Bo }}$ $f_{i j}^{(0), \mathrm{cl}}$ in the decompositions (3.31) used in $\mathcal{J}_{i}^{(1)}\left(f^{(0)}\right)$ are replaced by $f_{i j}^{(0), \text { sy }}-f_{i j}^{(0), \mathrm{cl}}$, where $f_{i j}^{(0), \text { Bo }}=f_{i}^{(0)}\left(\boldsymbol{x}_{i}^{\prime}, t\right) f_{j}^{(0)}\left(\boldsymbol{x}_{j}^{\prime}, t\right)$ and $f_{i j}^{(0), \text { sy }}=g_{i j}\left(r_{i j}\right) f_{i}^{(0)}\left(\boldsymbol{x}_{i}, t\right) f_{j}^{(0)}\left(\boldsymbol{x}_{j}, t\right)$. The deviations arising from the differences between the Bogoliubov zeroth order distribution functions $f_{i j}^{(0), \text { Bo }}$ and the symmetrized zeroth order distribution functions $f_{i j}^{(0), \text { sy }}$ will be shown to be negligible in some regime in Section 6.3. Using the symmetrized pair distribution functions $f_{i j}^{(0) \text {,sy }}$, the third order corrections in $\mathcal{J}_{i}^{(1)}\left(f^{(0)}\right)$ are then obtained in the form

$$
\Psi_{i}^{\mathrm{P}, 3}=\sum_{j \in \mathfrak{S}} \frac{1}{6 f_{i}^{(0)}} \int \theta_{i j} \partial_{\left(\mathbf{r}_{i}, \mathbf{r}_{j}\right)}^{3} f_{i j}^{(0), \text { sy }}:\left(0, \mathbf{r}_{i j}\right)^{\otimes 3} \mathrm{~d} \boldsymbol{x}_{j},
$$

so that

$$
\Psi_{i}^{\mathrm{P}, 3}=\sum_{j \in \mathfrak{S}} \frac{1}{6} \int \frac{\varphi_{i j}^{\prime}\left(r_{i j}\right)}{r_{i j}} \mathbf{r}_{i j} \cdot \frac{\left(\boldsymbol{c}_{i}-\boldsymbol{v}\right)}{k_{\mathrm{B}} T} g_{i j} \partial_{\mathbf{r}_{j}}^{3} n_{j}^{(0)}: \mathbf{r}_{i j}^{\otimes 3} \mathrm{~d} \mathbf{r}_{i j} .
$$

After some algebra, using the identity (F.5) of Appendix F, as well as the definition (5.2), it is obtained that

$$
\Psi_{i}^{\mathrm{P}, 3}=\sum_{j \in \mathfrak{S}} \frac{\varkappa_{i j}}{k_{\mathrm{B}} T}\left(\boldsymbol{c}_{i}-\boldsymbol{v}\right) \cdot \nabla \Delta n_{j}
$$

Combining the results without capillarity derived in Appendix D as well as the extra contributions in $\Psi_{i}^{\mathrm{K}, 3}$ and that of $\Psi_{i}^{\mathrm{P}, 3}$, we finally obtain new right hand sides $\psi_{i}^{(1)}$ with the same structure as (4.21) with unchanged functions $\psi_{i}^{\eta}, \psi_{i}^{\kappa}, \psi^{D_{j}}, j \in \mathfrak{S}$, and $\psi_{i}^{\widehat{\lambda}}$, but with the force terms

$$
\boldsymbol{X}_{i}=n_{i} k_{\mathrm{B}} T\left(\nabla\left(\frac{g_{i}^{\mathrm{u}}}{k_{\mathrm{B}} T}\right)-\sum_{j \in \mathfrak{S}} \frac{\varkappa_{i j}}{k_{\mathrm{B}} T} \nabla \Delta n_{j}\right)
$$

These force terms include both the thermodynamics $\boldsymbol{X}_{i}^{\text {th }}$ and Cahn-Hilliard $\boldsymbol{X}_{i}^{\text {ch }}$ contributions, generalizing (4.22). These force terms $\boldsymbol{X}_{i}$ also coincide with those obtained from rational thermodynamics in (2.16) and (2.17). The corresponding perturbed distribution functions $\phi^{(1)}=\left(\phi_{i}^{(1)}\right)_{i \in \mathfrak{S}}$ also satisfy Enskog type constraints that are found to be similar to that of normal diffusion processes as detailed in Appendix E. Therefore, we still have the expansions (4.21) and (4.32) with the new forces (6.9) but with unchanged functions $\phi_{i}^{\eta}, \phi_{i}^{\kappa}, \phi^{D_{j}}, j \in \mathfrak{S}$, and $\phi_{i}^{\widehat{\lambda}}$. We have thus recovered the Cahn-Hilliard force terms $\boldsymbol{X}_{i}^{\text {ch }}$ in diffusive fluxes and we must now investigate diffuse interface terms in first order conservation equations.

\subsection{Conservation equations at first order}

The conservation equations at first order are obtained by adding all first order contributions to the already derived conservation equations at the Euler level.

In order to investigate the extra capillary terms arising at first order of the Enskog expansion we use the symmetrized pair nonequilibrium distribution functions in the form

$$
f_{i j}^{\mathrm{sy}}=f_{i}\left(\mathbf{r}_{i}, \boldsymbol{c}_{i}, t\right) f_{j}\left(\mathbf{r}_{j}, \boldsymbol{c}_{j}, t\right) g_{i j}\left(\overline{\mathbf{r}}_{i, j}, r_{i j}\right), \quad i, j \in \mathfrak{S},
$$

where $g_{i j}$ is the correlation function (4.6) evaluated at a symmetric average $\overline{\mathbf{r}}_{i, j}$ of $\mathbf{r}_{i}$ and $\mathbf{r}_{j}$. This distribution first coincide with the zeroth order distribution $f_{i j}^{(0) \text {,sy }}$ when evaluated for Maxwellian distribution, it is also symmetric in its arguments and the 
limit for large $\mathbf{r}_{i j}$ is the natural uncorrelated nonequilibrium pair distribution function $f_{i}\left(\mathbf{r}_{i}, \boldsymbol{c}_{i}, t\right) f_{i}\left(\mathbf{r}_{j}, \boldsymbol{c}_{j}, t\right)$. The Euler equations for capillary fluids have also been evaluated by using $f_{i j}^{(0), \text { sy }}$. The correlation functions $g_{i j}\left(\overline{\mathbf{r}}_{i, j}, r_{i j}\right)$ are also simplified in the form $g_{i j}\left(\mathbf{r}_{i}, r_{i j}\right)$ as in the zeroth order derivation, and will be denoted by $g_{i j}\left(r_{i j}\right)$. In order to derive the macroscopic equations at first order, the symmetrized pair distribution functions $f_{i j}^{\text {sy }}$ are then used with $f_{i}=f_{i}^{(0)}+f_{i}^{(0)} \phi_{i}^{(1)}, i \in \mathfrak{S}$, with the general balance equations established in Section 3.2, using again higher order Taylor expansions. Denoting Bogoliubov pair distribution functions (3.30) by $f_{i j}^{\mathrm{Bo}}=f_{i}\left(\mathbf{r}_{i}^{\prime}, \boldsymbol{c}_{i}^{\prime}\right) f_{j}\left(\mathbf{r}_{j}^{\prime}, \boldsymbol{c}_{j}^{\prime}\right)$, the deviations arising from the difference $f_{i j}^{\mathrm{Bo}}-f_{i j}^{\mathrm{sy}}$ are addressed in the next section. In addition, we only need to consider the higher order terms in the Taylor expansions in order to evaluate the capillary contributions to the transport fluxes since the lower order terms have already been taken into account.

We first consider the tensor $\mathcal{P}^{\mathrm{P}}$ and look for new extra terms with two spatial derivatives arising from the first order Enskog contributions. The new terms - at first order of Enskog expansion - are in the form

$$
-\sum_{i, j \in \mathfrak{S}} \frac{1}{2} \int \frac{\varphi_{i j}^{\prime}\left(r_{i j}\right)}{r_{i j}} g_{i j}\left(r_{i j}\right) \mathbf{r}_{i j} \otimes \mathbf{r}_{i j} f_{i}^{(0)}\left(\mathbf{r}_{i}^{\alpha}\right) f_{j}^{(0)}\left(\mathbf{r}_{j}^{\alpha}\right)\left(\phi_{i}^{(1)}\left(\mathbf{r}_{i}^{\alpha}\right)+\phi_{j}^{(1)}\left(\mathbf{r}_{j}^{\alpha}\right)\right) \mathrm{d} \alpha \mathrm{d} \boldsymbol{c}_{i} \mathrm{~d} \mathbf{r}_{i j} \mathrm{~d} \boldsymbol{c}_{j},
$$

where we have denoted $\mathbf{r}_{i}^{\alpha}=\mathbf{r}_{i}-(1-\alpha) \mathbf{r}_{i j}$ and $\mathbf{r}_{j}^{\alpha}=\mathbf{r}_{i}+\alpha \mathbf{r}_{i j}$ and have left implicit the dependence on the velocities $\boldsymbol{c}_{i}$ and $\boldsymbol{c}_{j}$ as well as on time. These extra terms vanish since the average value with respect to $\boldsymbol{c}_{i}$ of $\phi_{i}^{(1)}$ at $\mathbf{r}_{i}^{\alpha}$ vanishes as well as the average value with respect to $\boldsymbol{c}_{j}$ of $\phi_{j}^{(1)}$ at $\mathbf{r}_{j}^{\alpha}$. As a consequence, there are no new contributions to the pressure tensor at first order, and in particular no extra capillary terms.

We now investigate the new contributions to the heat fluxes $\mathcal{Q}_{1}^{\mathrm{P}}$ and $\mathcal{Q}_{2}^{\mathrm{p}}$ at first order of Enskog expansion as well as those arising from the internal energy $\mathcal{E}$. Indeed, even though there is no first order corrector to the internal energy $\mathcal{E}$, by definition of the Enskog's constraints, there is nevertheless a first order corrector when transforming $\mathcal{E}^{\mathrm{ex}}=$ $-\sum_{i, j \in \mathfrak{S}} \frac{1}{2} \varkappa_{i j} n_{i} \Delta n_{j}$ into $\overline{\mathcal{E}}^{\mathrm{ex}}=\sum_{i, j \in \mathfrak{S}} \frac{1}{2} \varkappa_{i j} \nabla n_{i} \cdot \nabla n_{j}$. At first order of Enskog expansion, we indeed have to use the first order governing equation $\partial_{t} n_{i}=-\boldsymbol{\nabla} \cdot\left(n_{i} \boldsymbol{v}\right)-\boldsymbol{\nabla} \cdot \mathcal{F}_{i}, i \in \mathfrak{S}$, instead of $\partial_{t} n_{i}=-\boldsymbol{\nabla} \cdot\left(n_{i} \boldsymbol{v}\right), i \in \mathfrak{S}$, where $\mathcal{F}_{i}=\int\left(\boldsymbol{c}_{i}-\boldsymbol{v}\right) f_{i}^{(0)} \phi^{(1)} \mathrm{d} \boldsymbol{c}_{i}$ is the diffusive flux of particles with $\overline{\mathcal{F}}_{i}=m_{i} \mathcal{F}_{i}$. The corrector $\Xi$, given in the general form by (5.10), yields new terms solely arising from the time derivatives. Since the time derivatives in $\Xi$ arise through the divergence of $-\frac{1}{2} \varkappa_{i j}\left(\partial_{t} n_{i}+v \cdot \nabla n_{i}\right) \nabla n_{j}$ and $-\frac{1}{2} \varkappa_{i j} n_{i} \boldsymbol{\nabla}\left(\partial_{t} n_{j}+v \cdot \nabla n_{j}\right)$, and keeping in mind that the capillarity coefficients $\varkappa_{i j}$ are taken to be constant, we obtain after some algebra that the corrector $\Xi$ is in the form $\Xi=\boldsymbol{\nabla} \cdot\left(\mathfrak{q}_{0}+\mathfrak{q}_{0}^{\prime}\right)$ with a new first-order corrective flux $\mathfrak{q}_{0}^{\prime}$ given by

$$
\mathfrak{q}_{0}^{\prime}=\sum_{i, j \in \mathfrak{S}} \frac{1}{2} \varkappa_{i j} \boldsymbol{\nabla} \cdot \mathcal{F}_{i} \nabla n_{j}+\sum_{i, j \in \mathfrak{S}} \frac{1}{2} \varkappa_{i j} n_{j} \boldsymbol{\nabla}\left(\boldsymbol{\nabla} \cdot \mathcal{F}_{i}\right)
$$

Concentrating then on the flux $\mathcal{Q}_{1}^{\mathrm{P}}$, we have to investigate extra terms with second order derivatives arising from

$$
\sum_{i, j \in \mathfrak{S}} \frac{1}{2} \int \varphi_{i j}\left(\boldsymbol{c}_{i}-\boldsymbol{v}\right) g_{i j} f_{i}^{(0)}\left(\mathbf{r}_{i}\right) f_{j}^{(0)}\left(\mathbf{r}_{j}\right)\left(\phi_{i}^{(1)}\left(\mathbf{r}_{i}\right)+\phi_{j}^{(1)}\left(\mathbf{r}_{j}\right)\right) \mathrm{d} \boldsymbol{c}_{i} \mathrm{~d} \mathbf{r}_{i j} \mathrm{~d} \boldsymbol{c}_{j} .
$$

Using the zero average constraint for $\phi_{j}^{(1)}$ at $\mathbf{r}_{j}$, using the definition of $\mathcal{F}_{i}$ as well as that of $\varkappa_{i j}$, this contribution may be rewritten in the form $\sum_{i, j \in \mathfrak{S}} \frac{1}{2} \int \varphi_{i j} g_{i j} n_{j}\left(\mathbf{r}_{j}\right) \mathcal{F}_{i}\left(\mathbf{r}_{i}\right) \mathrm{d} \mathbf{r}_{i j}$. 
Using a Taylor expansion of $n_{j}\left(\mathbf{r}_{j}\right)$, the diffuse interface extra second order derivatives terms are finally obtained in the form

$$
\mathfrak{q}_{1}^{\prime}=-\sum_{i, j \in \mathfrak{S}} \frac{1}{2} \varkappa_{i j} \Delta n_{j} \mathcal{F}_{i}
$$

whereas the zeroth order terms in (6.12) are taken into account in the standard dissipative heat flux.

Concentrating then on the flux $\mathcal{Q}_{2}^{\text {p }}$, we must extract second derivative terms from the contributions

$$
\begin{aligned}
-\sum_{i, j \in \mathfrak{S}} \frac{1}{4} \int \frac{\varphi_{i j}^{\prime}\left(r_{i j}\right)}{r_{i j}} g_{i j}\left(r_{i j}\right) \mathbf{r}_{i j} \mathbf{r}_{i j} \cdot\left(\boldsymbol{c}_{i}-\boldsymbol{v}+\boldsymbol{c}_{j}-\boldsymbol{v}\right) \\
f_{i}^{(0)}\left(\mathbf{r}_{i}^{\alpha}\right) f_{j}^{(0)}\left(\mathbf{r}_{j}^{\alpha}\right)\left(\phi_{i}^{(1)}\left(\mathbf{r}_{i}^{\alpha}\right)+\phi_{j}^{(1)}\left(\mathbf{r}_{j}^{\alpha}\right)\right) \mathrm{d} \alpha \mathrm{d} \boldsymbol{c}_{i} \mathrm{~d} \mathbf{r}_{i j} \mathrm{~d} \boldsymbol{c}_{j} .
\end{aligned}
$$

Using the definition of diffusive fluxes, these terms may be rewritten

$$
-\sum_{i, j \in \mathfrak{S}} \frac{1}{4} \int \frac{\varphi_{i j}^{\prime}\left(r_{i j}\right)}{r_{i j}} g_{i j}\left(r_{i j}\right) \mathbf{r}_{i j} \mathbf{r}_{i j} \cdot\left(n_{j}\left(\mathbf{r}_{j}^{\alpha}\right) \mathcal{F}_{i}\left(\mathbf{r}_{i}^{\alpha}\right)+n_{i}\left(\mathbf{r}_{i}^{\alpha}\right) \mathcal{F}_{j}\left(\mathbf{r}_{j}^{\alpha}\right)\right) \mathrm{d} \alpha \mathrm{d} \mathbf{r}_{i j}
$$

We next use Taylor expansions for both number densities and diffusion fluxes and extract all the diffuse interface terms with two spatial derivatives. Considering first the integrands associated with the second order derivatives of number densities in (6.14), we obtain a corrector term in the form

$$
\mathfrak{q}_{2}^{\prime}=-\sum_{i, j \in \mathfrak{S}} \frac{1}{4} \int \frac{\varphi_{i j}^{\prime}\left(r_{i j}\right)}{r_{i j}} g_{i j}\left(r_{i j}\right) \mathbf{r}_{i j} \mathbf{r}_{i j} \cdot \frac{1}{2}\left(\nabla^{2} n_{j}:\left(\delta \mathbf{r}_{j}^{\alpha}\right)^{\otimes 2} \mathcal{F}_{i}+\nabla^{2} n_{i}:\left(\delta \mathbf{r}_{i}^{\alpha}\right)^{\otimes 2} \mathcal{F}_{j}\right) \mathrm{d} \alpha \mathrm{d} \mathbf{r}_{i j}
$$

where for any vector $\boldsymbol{z}$ the tensor $\boldsymbol{z} \otimes \boldsymbol{z}$ is also denoted for short by $\boldsymbol{z}^{\otimes 2}$. We may then integrate with respect to $\alpha$ by using $\int\left(\delta \mathbf{r}_{j}^{\alpha}\right)^{\otimes 2} \mathrm{~d} \alpha=\frac{1}{3} \mathbf{r}_{i j} \otimes \mathbf{r}_{i j}$ as well as $\int\left(\delta \mathbf{r}_{i}^{\alpha}\right)^{\otimes 2} \mathrm{~d} \alpha=$ $\frac{1}{3} \mathbf{r}_{i j} \otimes \mathbf{r}_{i j}$ and this yields

$$
\mathfrak{q}_{2}^{\prime}=-\sum_{i, j \in \mathfrak{S}} \frac{1}{12} \int \frac{\varphi_{i j}^{\prime}\left(r_{i j}\right)}{r_{i j}} g_{i j}\left(r_{i j}\right) \mathbf{r}_{i j} \mathbf{r}_{i j} \cdot \frac{1}{2}\left(\nabla^{2} n_{j}:\left(\mathbf{r}_{i j}\right)^{\otimes 2} \mathcal{F}_{i}+\nabla^{2} n_{i}:\left(\mathbf{r}_{i j}\right)^{\otimes 2} \mathcal{F}_{j}\right) \mathrm{d} \mathbf{r}_{i j} .
$$

Symmetrizing the sum and using the differential identity (F.3) we then obtain that $\mathfrak{q}_{2}^{\prime}$ is in the form

$$
\mathfrak{q}_{2}^{\prime}=-\sum_{i, j \in \mathfrak{S}} \frac{\varkappa_{i j}}{6}\left(2 \nabla^{2} n_{j}+\Delta n_{j} \boldsymbol{I}\right) \cdot \mathcal{F}_{i}
$$

with the cohesion coefficients $\varkappa_{i j}, i, j \in \mathfrak{S}$, given by (5.2). Considering then the integrands arising from products of first order derivatives of number densities and diffusive fluxes in (6.14) we obtain an extra corrector term in the form

$$
\mathfrak{q}_{3}^{\prime}=-\sum_{i, j \in \mathfrak{S}} \frac{1}{4} \int \frac{\varphi_{i j}^{\prime}\left(r_{i j}\right)}{r_{i j}} g_{i j}\left(r_{i j}\right) \mathbf{r}_{i j} \mathbf{r}_{i j} \cdot\left(\nabla n_{j} \cdot \delta \mathbf{r}_{j}^{\alpha} \nabla \mathcal{F}_{i} \cdot \delta \mathbf{r}_{i}^{\alpha}+\nabla n_{i} \cdot \delta \mathbf{r}_{i}^{\alpha} \nabla \mathcal{F}_{j} \cdot \delta \mathbf{r}_{j}^{\alpha}\right) \mathrm{d} \alpha \mathrm{d} \mathbf{r}_{i j}
$$

We may integrate with respect to $\alpha$ by using $\int\left(\delta \mathbf{r}_{i}^{\alpha}\right) \otimes\left(\delta \mathbf{r}_{j}^{\alpha}\right) \mathrm{d} \alpha=-\frac{1}{6} \mathbf{r}_{i j} \otimes \mathbf{r}_{i j}$ as well as $\int\left(\delta \mathbf{r}_{j}^{\alpha}\right) \otimes\left(\delta \mathbf{r}_{i}^{\alpha}\right) \mathrm{d} \alpha=-\frac{1}{6} \mathbf{r}_{i j} \otimes \mathbf{r}_{i j}$ and this yields

$$
\mathfrak{q}_{3}^{\prime}=\sum_{i, j \in \mathfrak{S}} \frac{1}{12} \int \frac{\varphi_{i j}^{\prime}\left(r_{i j}\right)}{r_{i j}} g_{i j}\left(r_{i j}\right) \mathbf{r}_{i j} \mathbf{r}_{i j} \cdot \nabla \mathcal{F}_{i} \cdot \mathbf{r}_{i j} \nabla n_{j} \cdot \mathbf{r}_{i j} \mathrm{~d} \mathbf{r}_{i j}
$$


We may next use again the differential identity (F.3) and after some algebra, it is obtained that

$$
\mathfrak{q}_{3}^{\prime}=\sum_{i, j \in \mathfrak{S}} \frac{1}{6} \varkappa_{i j}\left(\nabla \mathcal{F}_{i}+\left(\nabla \mathcal{F}_{i}\right)^{t}+\nabla \cdot \mathcal{F}_{i} \boldsymbol{I}\right) \cdot \nabla n_{j}
$$

Considering finally the integrands involving second derivatives of diffusive fluxes in (6.14) we obtain an extra corrector term in the form

$$
\mathfrak{q}_{4}^{\prime}=-\sum_{i, j \in \mathfrak{S}} \frac{1}{4} \int \frac{\varphi_{i j}^{\prime}\left(r_{i j}\right)}{r_{i j}} g_{i j}\left(r_{i j}\right) \mathbf{r}_{i j} \mathbf{r}_{i j} \cdot \frac{1}{2}\left(n_{j} \boldsymbol{\nabla}^{2} \mathcal{F}_{i}:\left(\delta \mathbf{r}_{i}^{\alpha}\right)^{\otimes 2}+n_{i} \boldsymbol{\nabla}^{2} \mathcal{F}_{j}:\left(\delta \mathbf{r}_{j}^{\alpha}\right)^{\otimes 2}\right) \mathrm{d} \alpha \mathrm{d} \mathbf{r}_{i j}
$$

We may then integrate with respect to $\alpha$ by using $\int\left(\delta \mathbf{r}_{j}^{\alpha}\right)^{\otimes 2} \mathrm{~d} \alpha=\frac{1}{3} \mathbf{r}_{i j} \otimes \mathbf{r}_{i j}$ as well as $\int\left(\delta \mathbf{r}_{i}^{\alpha}\right)^{\otimes 2} \mathrm{~d} \alpha=\frac{1}{3} \mathbf{r}_{i j} \otimes \mathbf{r}_{i j}$ and this yields

$$
\mathfrak{q}_{4}^{\prime}=-\sum_{i, j \in \mathfrak{S}} \frac{1}{12} \int \frac{\varphi_{i j}^{\prime}\left(r_{i j}\right)}{r_{i j}} g_{i j}\left(r_{i j}\right) \mathbf{r}_{i j} \mathbf{r}_{i j} \cdot \frac{1}{2}\left(n_{j} \nabla^{2} \mathcal{F}_{i}:\left(\mathbf{r}_{i j}\right)^{\otimes 2}+n_{i} \boldsymbol{\nabla}^{2} \mathcal{F}_{j}:\left(\mathbf{r}_{i j}\right)^{\otimes 2}\right) \mathrm{d} \mathbf{r}_{i j} .
$$

Using then the identity (F.6) it is obtained after some algebra that

$$
\mathfrak{q}_{4}^{\prime}=-\sum_{i, j \in \mathfrak{S}} \frac{1}{6} \varkappa_{i j}\left(\Delta \mathcal{F}_{i}+2 \nabla\left(\nabla \cdot \mathcal{F}_{i}\right)\right) n_{j}
$$

The new capillary heat flux at first order $\mathcal{Q}^{\text {ex }}$ may now be evaluated from

$$
\mathcal{Q}^{\text {ex }}=\mathfrak{q}_{0}^{\prime}+\mathfrak{q}_{1}^{\prime}+\mathfrak{q}_{2}^{\prime}+\mathfrak{q}_{3}^{\prime}+\mathfrak{q}_{4}^{\prime},
$$

with the terms evaluated from (6.11), (6.13), (6.15), (6.16), and (6.17). The extra heat flux $\mathcal{Q}^{\text {ex }}$ is thus given by

$$
\begin{aligned}
\mathcal{Q}^{\text {ex }}= & \sum_{i, j \in \mathfrak{S}} \frac{1}{2} \varkappa_{i j} \boldsymbol{\nabla} \cdot \mathcal{F}_{i} \nabla n_{j}+\sum_{i, j \in \mathfrak{S}} \frac{1}{2} \varkappa_{i j} n_{j} \boldsymbol{\nabla}\left(\boldsymbol{\nabla} \cdot \mathcal{F}_{i}\right)-\sum_{i, j \in \mathfrak{S}} \frac{1}{2} \varkappa_{i j} \Delta n_{j} \mathcal{F}_{i} \\
& -\sum_{i, j \in \mathfrak{S}} \frac{1}{6} \varkappa_{i j}\left(2 \nabla^{2} n_{j}+\Delta n_{j} \boldsymbol{I}\right) \cdot \mathcal{F}_{i}+\sum_{i, j \in \mathfrak{S}} \frac{1}{6} \varkappa_{i j}\left(\nabla \mathcal{F}_{i}+\left(\boldsymbol{\nabla} \mathcal{F}_{i}\right)^{t}+\nabla \cdot \mathcal{F}_{i} \boldsymbol{I}\right) \cdot \boldsymbol{\nabla} n_{j} \\
& -\sum_{i, j \in \mathfrak{S}} \frac{1}{6} \varkappa_{i j}\left(\Delta \mathcal{F}_{i}+2 \boldsymbol{\nabla}\left(\boldsymbol{\nabla} \cdot \mathcal{F}_{i}\right)\right) n_{j} .
\end{aligned}
$$

In order to simplify this expression we seek an equivalent expression $\overline{\mathcal{Q}}^{\text {'ex }}$ of $\mathcal{Q}^{\text {'ex }}$, that is, we seek a flux $\overline{\mathcal{Q}}^{\text {'ex }}$ such that

$$
\nabla \cdot \mathcal{Q}^{\mathrm{ex}}=\nabla \cdot \overline{\mathcal{Q}}^{\mathrm{ex}}
$$

To this aim, we use the identity (F.10) in order to eliminate the contributions involving $\nabla^{2} n_{j} \cdot \mathcal{F}_{i}$ and regroup similar terms and this yields

$$
\begin{aligned}
\boldsymbol{\nabla} \cdot \mathcal{Q}^{\prime \mathrm{ex}}= & \nabla \cdot\left(-\sum_{i, j \in \mathfrak{S}} \varkappa_{i j} \Delta n_{j} \mathcal{F}_{i}+\sum_{i, j \in \mathfrak{S}} \varkappa_{i j} \boldsymbol{\nabla} \cdot \mathcal{F}_{i} \nabla n_{j}-\sum_{i, j \in \mathfrak{S}} \frac{1}{6} \varkappa_{i j} \nabla \mathcal{F}_{i} \cdot \nabla n_{j}\right. \\
& \left.+\sum_{i, j \in \mathfrak{S}} \frac{1}{6} \varkappa_{i j}\left(\nabla \mathcal{F}_{i}\right)^{t} \cdot \nabla n_{j}+\sum_{i, j \in \mathfrak{S}} \frac{1}{6} \varkappa_{i j} n_{j} \boldsymbol{\nabla}\left(\boldsymbol{\nabla} \cdot \mathcal{F}_{i}\right)-\sum_{i, j \in \mathfrak{S}} \frac{1}{6} \varkappa_{i j} n_{j} \Delta \mathcal{F}_{i}\right) .
\end{aligned}
$$


Using then the differential identity (F.11), for each species pair $(i, j)$, the divergence of the third to sixth terms (four terms) in the right hand side vanishes, and we obtain that

$$
\boldsymbol{\nabla} \cdot \mathcal{Q}^{\mathrm{ex}}=\nabla \cdot\left(-\sum_{i, j \in \mathfrak{S}} \varkappa_{i j} \Delta n_{j} \mathcal{F}_{i}+\sum_{i, j \in \mathfrak{S}} \varkappa_{i j} \nabla \cdot \mathcal{F}_{i} \nabla n_{j}\right)
$$

and finally that

$$
\overline{\mathcal{Q}}^{\text {'ex }}=-\sum_{i, j \in \mathfrak{S}} \varkappa_{i j} \Delta n_{j} \mathcal{F}_{i}+\sum_{i, j \in \mathfrak{S}} \varkappa_{i j} \nabla \cdot \mathcal{F}_{i} \nabla n_{j}
$$

Using then the mass based diffuse interface coefficient $\bar{\varkappa}_{i j}=\varkappa_{i j} / m_{i} m_{j}$ with the relations $\rho_{i}=m_{i} n_{i}$ and $\overline{\mathcal{F}}_{i}=m_{i} \mathcal{F}_{i}$, we may rewrite the extra first order heat flux $\overline{\mathcal{Q}}^{\text {'ex }}$ in the form $-\sum_{i \in \mathfrak{S}} \nabla \cdot \bar{\gamma}_{i} \overline{\mathcal{F}}_{i}+\sum_{i \in \mathfrak{S}} \bar{\gamma}_{i} \boldsymbol{\nabla} \cdot \overline{\mathcal{F}}_{i}$ so that $\overline{\mathcal{Q}}^{\text {'ex }}$ coincides with $\mathcal{Q}^{\mathrm{CD}}$. A key point in the simplification of the capillary-diffusive flux was the use of the modified energy density (5.9) instead of (5.8), as already observed at zeroth order.

In summary, the generalized Korteweg tensor (2.18), the generalized Dunn and Serrin heat flux (2.19), the cohesive-dissipative heat flux (2.20), the energy density (2.7), the Cahn-Hilliard diffusive fluxes, as well as nonideal fluid thermodynamics have been recovered from the kinetic theory.

\subsection{Agreement with Bogoliubov distributions}

The higher order diffuse interface contributions in the conservation equations and the linearized equations have been derived by using the natural symmetrized pair distribution functions $f_{i j}^{\text {sy }}, i, j \in \mathfrak{S}$. More specifically, the Euler/van der Waals equations including the generalized Korteweg tensor (2.18), the generalized Dunn and Serrin heat flux (2.19), and the energy density (2.7) have been derived by using the zeroth order $f_{i j}^{(0), \text { sy }}$ pair distribution functions. Similarly, the Cahn-Hilliard diffusive fluxes and the capillary-diffusive heat flux (2.20) have been obtained by using the symmetrized pair distribution functions $f_{i j}^{\text {sy }}$. The symmetrized distributions are natural and Bogoliubov pair distribution functions are unfortunately not practical as typically shown by the explicit use of streaming operators in the dense gas mixture collision integrals $\overline{\mathfrak{a}}_{i j}^{\eta}, \overline{\mathfrak{a}}_{i j}^{\kappa}, \overline{\mathfrak{a}}_{i j}^{\widehat{\lambda}}$ of the linearized right hand sides (4.23)-(4.27). Nevertheless, Bogoliubov pair distribution functions have been used in order to evaluated the normal diffusive fluxes following Cohen et al. [50]. As a consequence, in order to establish that the model derived with the symmetrized distributions is in agreement with Bogoliubov distributions, we have to establish that the deviations in diffuse interface terms arising from the difference of pair distribution functions may be neglected in some regime. Since Bogoliubov pair distribution functions are unfortunately not practical, their moments cannot be evaluated, and it is inevitable to introduce some approximations.

We investigate the deviations in diffuse interfaces terms arising from the differences $f_{i j}^{\mathrm{Bo}}-f_{i j}^{\mathrm{sy}}$ in a regime where the dominant terms arise from the density derivatives and we make use of hard potential approximations. That is, we establish that the deviations arising from $f_{i j}^{\mathrm{Bo}}-f_{i j}^{\mathrm{sy}}$ may be neglected in a regime where temperature gradients and relative Mach numbers inside interphase fronts are small. To this aim, we introduce a characteristic length of temperature gradients $l_{\nabla T}^{\star}$, a characteristic length of velocity gradients $l_{\nabla v}^{\star}$, a typical macroscopic velocity variation inside interphase fronts $\delta v^{\star}$, and the corresponding relative Mach number $\mathrm{Ma}^{\star}=\delta v^{\star} / \sqrt{k_{\mathrm{B}} T^{\star} / m^{\star}}$. The Bogoliubov corrections are then estimated in a regime where density gradients dominate the dynamics of 
interphase fronts, that is, in the regime where

$$
\left(\frac{\sigma^{\star}}{r^{\star}}\right)^{3}\left(\frac{\sigma^{\star}}{l_{\nabla n}^{\star}}\right)\left(\frac{\sigma^{\star}}{l_{\nabla T}^{\star}}\right), \ll 1,
$$

and

$$
\left(\frac{\sigma^{\star}}{r^{\star}}\right)^{3}\left(\frac{\sigma^{\star}}{l_{\nabla n}^{\star}}\right)\left(\frac{\sigma^{\star}}{l_{\nabla v}^{\star}}\right)\left(\frac{\delta v^{\star}}{\sqrt{k_{\mathrm{B}} T^{\star} / m^{\star}}}\right) \ll 1 .
$$

The first condition (6.19) insure that all terms in the form $\varkappa_{i j} n_{i} \nabla n_{j} \cdot \nabla T$ are negligible with respect to the density gradient terms $\varkappa_{i j} \nabla n_{i} \cdot \nabla n_{j}$ within interphase fronts, and a similar condition holds for the gradient of these quantities. Similarly, the second condition (6.20) insure that all terms in the form $\varkappa_{i j} n_{i} \boldsymbol{\nabla} n_{j} \cdot \boldsymbol{\nabla} \boldsymbol{v} / \sqrt{k_{\mathrm{B}} T / m}$ are negligible with respect to the density gradient terms $\varkappa_{i j} \boldsymbol{\nabla} n_{i} \cdot \boldsymbol{\nabla} n_{j}$ within interphase fronts, and the same rule applies to gradients of these quantities.

We may next introduce the decomposition

$$
f_{i}^{(0)}=n_{i} \widetilde{f}_{i}^{(0)}, \quad \widetilde{f}_{i}^{(0)}=\left(\frac{m_{i}}{2 \pi k_{\mathrm{B}} T}\right)^{\frac{3}{2}} \exp \left(-\frac{m_{i}\left|\boldsymbol{c}_{i}-\boldsymbol{v}\right|^{2}}{2 k_{\mathrm{B}} T}\right),
$$

such that

$$
\begin{aligned}
\nabla f_{i}^{(0)} & =\nabla n_{i} \widetilde{f}_{i}^{(0)}+n_{i} \boldsymbol{\nabla} \widetilde{f}_{i}^{(0)}, \\
\nabla^{2} f_{i}^{(0)} & =\nabla^{2} n_{i} \widetilde{f}_{i}^{(0)}+\nabla n_{i} \otimes \nabla \widetilde{f}_{i}^{(0)}+\nabla \widetilde{f}_{i}^{(0)} \otimes \nabla n_{i}+n_{i} \nabla^{2} \widetilde{f}_{i}^{(0)} .
\end{aligned}
$$

From the expression

$$
\boldsymbol{\nabla} \log \widetilde{f}_{i}^{(0)}=\frac{m_{i}}{k_{\mathrm{B}} T}\left(\boldsymbol{c}_{i}-\boldsymbol{v}\right) \cdot \boldsymbol{\nabla} \boldsymbol{v}-\left(\frac{3}{2}-\frac{m_{i}\left|\boldsymbol{c}_{i}-\boldsymbol{v}\right|^{2}}{2 k_{\mathrm{B}} T}\right) \frac{\boldsymbol{\nabla} T}{T},
$$

we then note that the ratio of the terms in $\nabla \log \widetilde{f}_{i}^{(0)}$ with respect to $\nabla \log n_{i}$ is of the order of $\frac{l_{\nabla n}}{l_{\nabla v}} \mathrm{Ma}^{\star}$ or $\frac{l_{\nabla n}}{l_{\nabla T}}$ in such a way that $\nabla \log n_{i}$ is the main contribution in $\nabla \log f_{i}^{(0)}$ inside interphase fronts where $\sigma^{\star} \approx l_{\nabla n}^{\star}, \sigma^{\star} \ll l_{\nabla T}^{\star}$ and $\sigma^{\star} \times \delta v^{\star} \ll l_{\nabla v}^{\star} \times\left(k_{\mathrm{B}} T^{\star} / m^{\star}\right)^{1 / 2}$. The conditions (6.19) and (6.20) thus insure that the main contributions in the gradients $\nabla f_{i}^{(0)}$ in interphase fronts are those associated with $\nabla n_{i}$.

The number densities $n_{i}, i \in \mathfrak{S}$, and the fluid velocity $\boldsymbol{v}$ are unchanged by correction terms concerning pair distribution functions but there is first a difference of internal energies in the form

$$
\delta^{\mathrm{Bo}} \mathcal{E}=\sum_{i, j \in \mathfrak{S}} \int \frac{1}{2} \varphi_{i j}\left(f_{i j}^{(0), \mathrm{Bo}}-f_{i j}^{(0), \mathrm{sy}}\right) \mathrm{d} \boldsymbol{c}_{i} \mathrm{~d} \boldsymbol{r}_{i j} \mathrm{~d} \boldsymbol{c}_{j},
$$

where we have denoted by $f_{i j}^{(0), \text { Bo }}=f_{i}^{(0)}\left(\mathbf{r}_{i}^{\prime}, \boldsymbol{c}_{i}^{\prime}\right) f_{j}^{(0)}\left(\mathbf{r}_{j}^{\prime}, \boldsymbol{c}_{j}^{\prime}\right)$ the zeroth order Bogoliubov distribution functions. With second order expansions of the pair distribution functions, the difference in internal energy reads

$$
\delta^{\mathrm{Bo} \mathcal{E}}=\sum_{i, j \in \mathfrak{S}} \frac{1}{4} \int \varphi_{i j}\left(\partial_{\left(\mathbf{r}_{i}, \mathbf{r}_{j}\right)}^{2} f_{i j}^{(0), \mathrm{Bo}}:\left(\delta \mathbf{r}_{i}, \delta \mathbf{r}_{j}\right)^{\otimes 2}-\partial_{\mathbf{r}_{j}}^{2} f_{i j}^{(0), \mathrm{sy}}: \mathbf{r}_{i j}^{\otimes 2}\right) \mathrm{d} \boldsymbol{c}_{i} \mathrm{~d} \mathbf{r}_{i j} \mathrm{~d} \boldsymbol{c}_{j}
$$

where for any vector $\boldsymbol{z} \in \mathbb{R}^{3}$ we denote for short by $\boldsymbol{z}^{\otimes 2}$ the tensor product $\boldsymbol{z} \otimes \boldsymbol{z}$ and where $\delta \mathbf{r}_{i}=\mathbf{r}_{i}^{\prime}-\mathbf{r}_{i}$ and $\delta \mathbf{r}_{j}=\mathbf{r}_{j}^{\prime}-\mathbf{r}_{j}$ have been defined in Section 3.4. The leading terms 
in $\delta^{\mathrm{Bo}} \mathcal{E}$ are thus

$$
\begin{aligned}
\sum_{i, j \in \mathfrak{S}} \frac{1}{4} \int \varphi_{i j} g_{i j} \widetilde{f}_{i}^{(0)} \widetilde{f}_{j}^{(0)}( & n_{j} \partial_{\mathbf{r}_{i}}^{2} n_{i}:\left(\delta \mathbf{r}_{i}\right)^{\otimes 2}+2 \partial_{\mathbf{r}_{i}} n_{i} \cdot \delta \mathbf{r}_{i} \partial_{\mathbf{r}_{j}} n_{j} \cdot \delta \mathbf{r}_{j} \\
& \left.+n_{i} \partial_{\mathbf{r}_{j}}^{2} n_{j}:\left(\delta \mathbf{r}_{j}\right)^{\otimes 2}-n_{i} \partial_{\mathbf{r}_{j}}^{2} n_{j}: \mathbf{r}_{i j}^{\otimes 2}\right) \mathrm{d} \boldsymbol{c}_{i} \mathrm{~d} \mathbf{r}_{i j} \mathrm{~d} \boldsymbol{c}_{j}
\end{aligned}
$$

and all these terms vanish from the integral relations (D.12) for hard potentials that are simply obtained by letting $\delta \mathbf{r}_{i}=0 \delta \mathbf{r}_{j}=\mathbf{r}_{i j}$ inside the integrals. The remaining correction terms are then given by

$$
\begin{aligned}
\sum_{i, j \in \mathfrak{S}} & \frac{1}{4} \int \varphi_{i j} g_{i j} n_{i} n_{j}\left(\partial_{\left(\mathbf{r}_{i}, \mathbf{r}_{j}\right)}^{2} \widetilde{f}_{i j}^{(0), \mathrm{Bo}}:\left(\delta \mathbf{r}_{i}, \delta \mathbf{r}_{j}\right)^{\otimes 2}-\partial_{\mathbf{r}_{j}}^{2} \widetilde{f}_{i j}^{(0), \mathrm{sy}}: \mathbf{r}_{i j}^{\otimes 2}\right) \mathrm{d} \boldsymbol{c}_{i} \mathrm{~d} \mathbf{r}_{i j} \mathrm{~d} \boldsymbol{c}_{j} \\
+ & \sum_{i, j \in \mathfrak{S}} \frac{1}{2} \int \varphi_{i j} g_{i j}\left(\left(n_{j} \partial_{\mathbf{r}_{i}} n_{i} \cdot \delta \mathbf{r}_{i}+n_{i} \partial_{\mathbf{r}_{j}} n_{j} \cdot \delta \mathbf{r}_{j}\right) \partial_{\left(\mathbf{r}_{i}, \mathbf{r}_{j}\right)} \widetilde{f}_{i j}^{(0), \mathrm{Bo}} \cdot\left(\delta \mathbf{r}_{i}, \delta \mathbf{r}_{j}\right)\right. \\
& \left.-\partial_{\mathbf{r}_{j}} n_{j} \cdot \mathbf{r}_{i j} \partial_{\mathbf{r}_{j}} \widetilde{f}_{i j}^{(0), \mathrm{sy}} \cdot \mathbf{r}_{i j}\right) \mathrm{d} \boldsymbol{c}_{i} \mathrm{~d} \mathbf{r}_{i j} \mathrm{~d} \boldsymbol{c}_{j},
\end{aligned}
$$

where $\widetilde{f}_{i j}^{(0), \text { Bo }}=\widetilde{f}_{i}^{(0)}\left(\mathbf{r}_{i}^{\prime}, \boldsymbol{c}_{i}^{\prime}\right) \widetilde{f}_{j}^{(0)}\left(\mathbf{r}_{j}^{\prime}, \boldsymbol{c}_{j}^{\prime}\right)$ and $\widetilde{f}_{i j}^{(0), s y}=g_{i j} \widetilde{f}_{i}^{(0)}\left(\mathbf{r}_{i}, \boldsymbol{c}_{i}\right) \widetilde{f}_{j}^{(0)}\left(\mathbf{r}_{j}, \boldsymbol{c}_{j}\right)$. These corrections are thus negligible since $\varkappa^{\star}=k_{\mathrm{B}} T^{\star} \sigma^{\star 5}$ as established in Section 5.3, and the

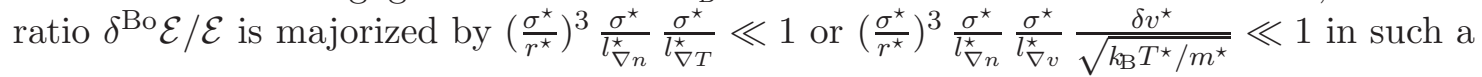
way that from (6.19) and (6.20) the correction term $\delta^{\text {Bo } \mathcal{E}}$ may be neglected.

The lengthy estimates for the deviations of the pressure tensor $\delta^{\mathrm{Bo}} \mathcal{P}=\delta^{\mathrm{Bo}} \mathcal{P}^{\mathrm{P}}$ and of the heat fluxes $\delta^{\text {Bo }} \mathcal{Q}_{1}^{\mathrm{P}}$ and $\delta^{\text {Bo }} \mathcal{Q}_{2}^{\mathrm{P}}$, arising from $f_{i j}^{(0) \text {, Bo }}-f_{i j}^{(0), \text { sy }}$, are discussed in Appendix G. These estimates are obtained in a similar way as for the energy with dominant terms vanishing under the hard potential approximation (D.11) and (D.12) and remaining terms involving gradients of $\widetilde{f}_{i}^{(0)}$ and $\widetilde{f}_{j}^{(0)}$ that are negligible under the regime (6.19) and (6.20). The result is that the corrections arising from $f_{i j}^{(0), \text { Bo }}-f_{i j}^{(0), \text { sy }}$ may be neglected and the first order corrections in the conservation equations, that are of multiplied by the Knudsen number, are also negligible. The Bogoliubov corrections in the conservation equations are thus negligible in the regime under consideration and it is legitimate to use $f_{i j}^{(0), \text { sy }}$ instead of $f_{i j}^{(0), \text { Bo }}$ in the derivation of the conservation equations. In order to complete the analysis, we now have to investigate the deviations arising from $f_{i j}^{(0), \text { Bo }}-f_{i j}^{(0) \text {,sy }}$ in the linearized equations

The differences in the right hand sides of the linearized equations $\delta^{\mathrm{Bo}} \psi_{i}$, arise from the material derivatives $\partial_{t} f_{i}^{(0)}+\boldsymbol{v} \cdot \partial_{\mathbf{r}_{i}} f_{i}^{(0)}$ and the perturbed source terms $\mathcal{J}_{i}^{(1)}$. From the estimates of the pressure tensor, the energy and the heat fluxes, the differences in $\partial_{t} f_{i}^{(0)}+\boldsymbol{v} \cdot \partial_{\mathbf{r}_{i}} f_{i}^{(0)}$ due to $f_{i j}^{(0), \text { Bo }}-f_{i j}^{(0), \text { sy }}$ in $\psi_{i}$ are negligible and we must solely examine the differences arising from the perturbed operators $\mathcal{J}_{i}^{(1)}$. We have introduced in (6.6) the decomposition $\frac{1}{f_{i}^{(0)}} \mathcal{J}_{i}^{(1)}\left(f^{(0)}\right)=\Psi_{i}^{\mathrm{P}, 1}+\Psi_{i}^{\mathrm{P}, 3}$ and the first order terms $\Psi_{i}^{\mathrm{P}, 1}=\sum_{j \in \mathfrak{S}} \frac{1}{f_{i}^{(0)}} \int \theta_{i j} \partial_{\left(\mathbf{r}_{i}, \mathbf{r}_{j}\right)} f_{i j}^{(0), \mathrm{Bo}} \cdot\left(\delta \mathbf{r}_{i}, \delta \mathbf{r}_{j}\right) \mathrm{d} \boldsymbol{x}_{j}$ have already been taken into account in the linearized equations. We only have to investigate the differences in the third order terms

$$
\delta^{\mathrm{Bo}} \Psi_{i}^{\mathrm{P}, 3}=\sum_{j \in \mathfrak{S}} \frac{1}{6 f_{i}^{(0)}} \int \theta_{i j}\left(\partial_{\left(\mathbf{r}_{i}, \mathbf{r}_{j}\right)}^{3} f_{i j}^{(0), \mathrm{Bo}}:\left(\delta \mathbf{r}_{i}, \delta \mathbf{r}_{j}\right)^{\otimes 3}-\partial_{\left(\mathbf{r}_{i}, \mathbf{r}_{j}\right)}^{3} f_{i j}^{(0), \mathrm{sy}}:\left(0, \mathbf{r}_{i j}\right)^{\otimes 3}\right) \mathrm{d} \boldsymbol{x}_{j}
$$


where $\partial_{\left(\mathbf{r}_{i}, \mathbf{r}_{j}\right)}$ denotes the differential operator with respect to the pair coordinates $\left(\mathbf{r}_{i}, \mathbf{r}_{j}\right)$, where $\delta \mathbf{r}_{i}=\mathbf{r}_{i}^{\prime}-\mathbf{r}_{i}=\mu_{j} \mathbf{r}_{i j}-\mu_{j} \mathbf{r}_{i j}^{\prime}$ and $\delta \mathbf{r}_{j}=\mathbf{r}_{j}^{\prime}-\mathbf{r}_{i}=\mu_{j} \mathbf{r}_{i j}+\mu_{i} \mathbf{r}_{i j}^{\prime}$, and with the differentials $\partial_{\left(\mathbf{r}_{i}, \mathbf{r}_{j}\right)}^{3} f_{i j}^{(0)}$ evaluated at $\left(\mathbf{r}_{i}, \mathbf{r}_{i}\right)$. All terms that are quadratic in the increments $\delta \mathbf{r}_{i}$ and $\delta \mathbf{r}_{j}$ indeed yield zero contributions in the expansion of $\mathcal{J}_{i}^{(1)}\left(f^{(0)}\right)$, being even with respect to $\mathbf{r}_{i j}$ and $\mathbf{r}_{i j}$, keeping in mind that $\theta_{i j}$ is even with respect to $\mathbf{r}_{i j}$. The higher order density derivatives contributions then vanish with the hard potentials approximation (D.11) and (D.12) as in the analysis of the energy. The remaining terms then involve derivatives of the rescaled Maxwellians $\widetilde{f}_{i}^{(0)}$ or $\widetilde{f}_{j}^{(0)}$ that are negligible in the regime under consideration (6.19) (6.20).

We conclude this section by presenting a more qualitative argument that relate the zeroth order pair distribution functions. The distribution $f_{i j}^{(0), \text { Bo }}=f_{i}^{(0)}\left(\mathbf{r}_{i}^{\prime}, \boldsymbol{c}_{i}^{\prime}\right) f_{j}^{(0)}\left(\mathbf{r}_{j}^{\prime}, \boldsymbol{c}_{j}^{\prime}\right)$ is first decomposed as

$$
f_{i j}^{(0), \mathrm{Bo}}=n_{i}\left(\mathbf{r}_{i}^{\prime}\right) n_{j}\left(\mathbf{r}_{j}^{\prime}\right) \widetilde{f}_{i}^{(0)}\left(\mathbf{r}_{i}^{\prime}, \boldsymbol{c}_{i}^{\prime}\right) \widetilde{f}_{j}^{(0)}\left(\mathbf{r}_{j}^{\prime}, \boldsymbol{c}_{j}^{\prime}\right),
$$

and in the regime under consideration we may write $T\left(\mathbf{r}_{i}\right)=T\left(\mathbf{r}_{i}^{\prime}\right)=T\left(\mathbf{r}_{j}\right)=T\left(\mathbf{r}_{j}^{\prime}\right)=$ $\bar{T}$ as well as $\boldsymbol{v}\left(\mathbf{r}_{i}\right)=\overline{\boldsymbol{v}}+\delta \boldsymbol{v}_{i}, \boldsymbol{v}\left(\mathbf{r}_{i}^{\prime}\right)=\overline{\boldsymbol{v}}+\delta \boldsymbol{v}_{i}^{\prime}, \boldsymbol{v}\left(\mathbf{r}_{j}\right)=\overline{\boldsymbol{v}}+\delta \boldsymbol{v}_{j}, \boldsymbol{v}\left(\mathbf{r}_{j}^{\prime}\right)=\overline{\boldsymbol{v}}+\delta \boldsymbol{v}_{j}^{\prime}$ where all relative Mach numbers $\delta \boldsymbol{v}_{i} \sqrt{m_{i} / 2 k_{\mathrm{B}} T(\mathbf{r})}, \delta \boldsymbol{v}_{i}^{\prime} \sqrt{m_{i} / 2 k_{\mathrm{B}} T(\mathbf{r})}, \delta \boldsymbol{v}_{j} \sqrt{m_{j} / 2 k_{\mathrm{B}} T(\mathbf{r})}$, and $\delta \boldsymbol{v}_{j}^{\prime} \sqrt{m_{j} / 2 k_{\mathrm{B}} T(\mathbf{r})}$, are negligible. The rescaled Maxellian distributions may thus be written

$$
\widetilde{f}_{i}^{(0)}(\mathbf{r}, \boldsymbol{c})=\left(\frac{m_{i}}{2 \pi k_{\mathrm{B}} \bar{T}}\right)^{\frac{3}{2}} \exp \left(-\frac{m_{i}|\boldsymbol{c}-\overline{\boldsymbol{v}}|^{2}}{2 k_{\mathrm{B}} \bar{T}}\right), \quad i \in \mathfrak{S},
$$

for any velocity $\boldsymbol{c}$, so that

$$
\widetilde{f}_{i}^{(0)}\left(\mathbf{r}_{i}^{\prime}, \boldsymbol{c}_{i}^{\prime}\right) \widetilde{f}_{i}^{(0)}\left(\mathbf{r}_{j}^{\prime}, \boldsymbol{c}_{j}^{\prime}\right)=\left(\frac{\sqrt{m_{i} m_{j}}}{2 \pi k_{\mathrm{B}} \bar{T}}\right)^{3} \exp \left(-\frac{m_{i}\left|\boldsymbol{c}_{i}^{\prime}-\overline{\boldsymbol{v}}\right|^{2}+m_{j}\left|\boldsymbol{c}_{j}^{\prime}-\overline{\boldsymbol{v}}\right|^{2}}{2 k_{\mathrm{B}} \bar{T}}\right) .
$$

Using the conservation of energy (3.29) and of momentum (3.28) we next obtain that

$$
\widetilde{f}_{i}^{(0)}\left(\mathbf{r}_{i}^{\prime}, \boldsymbol{c}_{i}^{\prime}\right) \widetilde{f}_{i}^{(0)}\left(\mathbf{r}_{j}^{\prime}, \boldsymbol{c}_{j}^{\prime}\right)=g_{i j}\left(\frac{\sqrt{m_{i} m_{j}}}{2 \pi k_{\mathrm{B}} \bar{T}}\right)^{3} \exp \left(-\frac{m_{i}\left|\boldsymbol{c}_{i}-\overline{\boldsymbol{v}}\right|^{2}+m_{j}\left|\boldsymbol{c}_{j}-\overline{\boldsymbol{v}}\right|^{2}}{2 k_{\mathrm{B}} \bar{T}}\right) .
$$

Using the same arguments for $\widetilde{f}_{i}^{(0)}\left(\mathbf{r}_{i}, \boldsymbol{c}_{i}\right) \widetilde{f}_{i}^{(0)}\left(\mathbf{r}_{j}, \boldsymbol{c}_{j}\right)$ then yields $\widetilde{f}_{i}^{(0)}\left(\mathbf{r}_{i}^{\prime}, \boldsymbol{c}_{i}^{\prime}\right) \widetilde{f}_{i}^{(0)}\left(\mathbf{r}_{j}^{\prime}, \boldsymbol{c}_{j}^{\prime}\right)=$ $g_{i j} \widetilde{f}_{i}^{(0)}\left(\mathbf{r}_{i}, \boldsymbol{c}_{i}\right) \widetilde{f}_{i}^{(0)}\left(\mathbf{r}_{j}, \boldsymbol{c}_{j}\right)$. We may then use the hard potential approximation for the number densities $n_{i}\left(\mathbf{r}_{i}^{\prime}\right) n_{j}\left(\mathbf{r}_{j}^{\prime}\right)=n_{i}\left(\mathbf{r}_{i}\right) n_{j}\left(\mathbf{r}_{j}\right)$ and this finally yields that, in the regime under consideration

$$
f_{i j}^{(0), \text { Bo }}=g_{i j} f_{i}^{(0)}\left(\mathbf{r}_{i}, \boldsymbol{c}_{i}\right) f_{j}^{(0)}\left(\mathbf{r}_{j}, \boldsymbol{c}_{j}\right)=f_{i j}^{(0), \text { sy }} .
$$

In summary, the equations derived with the symmetrized pair distribution functions also agree with Bogoliubov distribution functions in a regime where density gradients dominate the dynamics of interphase fronts and when interaction potentials are hard.

\section{Comparison and discussion}

We discuss in this section the model derived from the kinetic theory, the other models derived from thermodynamics in the literature as well as the domain of validity of the resulting equations. 


\subsection{Derivation from thermodynamics}

A Cahn-Hilliard fluid model has been derived from the kinetic theory of dense gas mixtures. The model, derived at the molecular level, is in full agreement with the model derived from rational thermodynamic in Section 2. In comparison with the equations governing nonideal fluids derived in Section 4, the Cahn-Hilliard fluid model involve various extra gradient terms. Such extra terms arise as gradient approximations of nonlocalities due to the perturbed kinetic operators $\mathcal{J}_{i}^{(1)}, i \in \mathfrak{S}$. At zeroth order of Enskog expansion, nonlocalities of Maxwellian distributions yield the Korteweg type tensor, the van der Waals/Cahn-Hilliard gradient energy terms as well as the Dunn and Serrin type heat flux. At first order of Enskog expansion, nonlocalities in Maxwellian distributions yield the Cahn-Hilliard diffusion force terms in the linearized equations whereas nonlocalities in the perturbed distribution functions yield the capillary-diffusive contributions in the heat flux.

An important task is now to compare the resulting model with those derived in previous work $[2,19,20,21,22,23]$. All terms are found to agree with the exception of the capillary-diffusive heat flux $\mathcal{Q}^{\mathrm{CD}}$ and the force terms $\boldsymbol{X}_{i}, i \in \mathfrak{S}$. More specifically, the kinetic theory yields an heat flux in the form

$$
\mathcal{Q}=\sum_{i \in \mathfrak{S}} \bar{\gamma}_{i} \rho_{i} \boldsymbol{\nabla} \cdot \boldsymbol{v}+\sum_{i \in \mathfrak{S}} \bar{\gamma}_{i} \boldsymbol{\nabla} \cdot \overline{\mathcal{F}}_{i}-\sum_{i \in \mathfrak{S}} \boldsymbol{\nabla} \cdot \bar{\gamma}_{i} \overline{\mathcal{F}}_{i}+\mathcal{Q}^{\mathrm{d}}
$$

and forces terms as

$$
\boldsymbol{X}_{i}=n_{i} k_{\mathrm{B}} T\left(\nabla \frac{g_{i}}{k_{\mathrm{B}} T}-\sum_{j \in \mathfrak{S}} \frac{\nabla \nabla \cdot\left(\varkappa_{i j} \nabla n_{j}\right)}{k_{\mathrm{B}} T}\right) .
$$

On the other hand, by using the expession (A.6) for the entropy production rate, the following heat flux and force terms are obtained

$$
\begin{gathered}
\mathcal{Q}^{\prime}=\sum_{i \in \mathfrak{S}} \bar{\gamma}_{i} \rho_{i} \boldsymbol{\nabla} \cdot \boldsymbol{v}+\sum_{i \in \mathfrak{S}} \bar{\gamma}_{i} \boldsymbol{\nabla} \cdot \overline{\mathcal{F}}_{i}+\mathcal{Q}^{\mathrm{d}}, \\
\boldsymbol{X}_{i}^{\prime}=n_{i} k_{\mathrm{B}} T \nabla\left(\frac{g_{i}}{k_{\mathrm{B}} T}-\sum_{j \in \mathfrak{S}} \frac{\boldsymbol{\nabla} \cdot\left(\varkappa_{i j} \boldsymbol{\nabla} n_{j}\right)}{k_{\mathrm{B}} T}\right),
\end{gathered}
$$

so that $\boldsymbol{X}_{i}^{\prime}-\boldsymbol{X}_{i}=n_{i} \boldsymbol{\nabla} \cdot \gamma_{i} \boldsymbol{\nabla} T / T$. These expressions have notably been derived by Anderson et al. [2], Verschueren [20] and Liu et al. [23]. Similarly, by using the expession (A.8) for the entropy production rate, the following heat flux and force terms are obtained

$$
\begin{gathered}
\mathcal{Q}^{\prime \prime}=\sum_{i \in \mathfrak{S}} \bar{\gamma}_{i} \rho_{i} \nabla \cdot \boldsymbol{v}+\mathcal{Q}^{\mathrm{d}}, \\
\boldsymbol{X}_{i}^{\prime \prime}=n_{i} k_{\mathrm{B}} T \nabla\left(\frac{g_{i}}{k_{\mathrm{B}} T}-\sum_{j \in \mathfrak{S}} \boldsymbol{\nabla} \cdot\left(\frac{\varkappa_{i j} \nabla n_{j}}{k_{\mathrm{B}} T}\right)\right),
\end{gathered}
$$

and then $\boldsymbol{X}_{i}^{\prime \prime}-\boldsymbol{X}_{i}=n_{i} T \boldsymbol{\nabla}\left(\bar{\gamma}_{i} \cdot \boldsymbol{\nabla} T / T^{2}\right)+n_{i} \boldsymbol{\nabla} \cdot \boldsymbol{\gamma}_{i} \boldsymbol{\nabla} T / T$. These expressions have notably been obtained by Falk [19], and by Alt and Pawlow [24] without convection phenomena.

We then first note that the models (7.3)-(7.4) and (7.5)-(7.6) exactly agree with (7.1)-(7.2) when all capillary coefficients are equal $\bar{\varkappa}_{i j}=\bar{\varkappa}_{\text {since then all differences }}$ automatically vanish from mass conservation constraints. Indeed, in this situation, the vectors $\bar{\gamma}_{i}$ are given by $\bar{\gamma}_{i}=\bar{\varkappa} \nabla \rho, i \in \mathfrak{S}$, and are all equal so that $\sum_{i \in \mathfrak{S}} \bar{\gamma}_{i} \boldsymbol{\nabla} \cdot \overline{\mathcal{F}}_{i}=$ 
$\bar{\varkappa} \nabla \rho \nabla \cdot\left(\sum_{i \in \mathfrak{S}} \overline{\mathcal{F}}_{i}\right)=0$ from the mass conservation constraint $\sum_{i \in \mathfrak{S}} \overline{\mathcal{F}}_{i}=0$ and similarly $\sum_{i \in \mathfrak{S}} \nabla \cdot \bar{\gamma}_{i} \overline{\mathcal{F}}_{i}=\nabla \bar{\varkappa} \nabla \rho \sum_{i \in \mathfrak{S}} \overline{\mathcal{F}}_{i}=0$. This was the model used in particular by Gaillard et al. [9] in order to simulate hydrogen-air transcritical flames. In this situation the capillary terms in the Cahn-Hilliard diffusive fluxes also vanish [9]. The resulting model may be seen as a generalization to mixtures of the van der Walls model with a simplified $\bar{\varkappa}|\boldsymbol{\nabla} \rho|^{2}$ gradient energy in the free energy [9].

More generally, we note that the models (7.3)-(7.4) and (7.5)-(7.6) essentially agree with (7.1)-(7.2) in the regimes under consideration. Indeed, the differences between the forces terms involve temperature gradients and the relative errors scale as $\left(\frac{\sigma^{\star}}{r^{\star}}\right)^{3} \frac{\sigma^{\star}}{l_{\nabla n}^{\star}} \frac{\sigma^{\star}}{l_{\nabla T}^{\star}} \ll$ 1 where $\sigma^{\star}$ denotes a typical collision diameter, $r^{\star}$ the average interparticle distance, $l_{\nabla n}^{\star}$ a characteristic length of density gradients, and $l_{\nabla T}^{\star}$ a characteristic length of velocity or temperature gradients, and may thus be neglected since temperature gradients remain modest in interphase fronts. For similar reasons, the first order contributions in the heat fluxes may be estimated to be negligible in the regime under consideration, keeping in mind that the force terms vanish at equilibrium interfaces. With these approximations, the three models (7.3)-(7.4), (7.5)-(7.6), and (7.1)-(7.2) are essentially similar.

On the other hand, various authors have further extracted the natural $1 / T$ factor in the thermodynamic fluxes. The resulting expression of the entropy production rate (A.9) then leads to the heat flux

$$
\mathcal{Q}=\sum_{i \in \mathfrak{S}} \bar{\gamma}_{i} \rho_{i} \boldsymbol{\nabla} \cdot \boldsymbol{v}+\sum_{i \in \mathfrak{S}} \bar{\gamma}_{i} \boldsymbol{\nabla} \cdot \overline{\mathcal{F}}_{i}-\sum_{i \in \mathfrak{S}}\left(\bar{g}_{i}-\nabla \cdot \bar{\gamma}_{i}\right) \overline{\mathcal{F}}_{i}+\mathcal{Q}^{\mathrm{d}}
$$

with an unphysical component $\sum_{i \in \mathfrak{S}} \bar{g}_{i} \overline{\mathcal{F}}_{i}$, and such fluxes have been considered in particular by Heida et al. [21] and Guo at al. [22]. Letting the capillary coefficient to zero, the resulting heat flux is in the form $\mathcal{Q}=-\sum_{i \in \mathfrak{S}} \bar{g}_{i} \overline{\mathcal{F}}_{i}+\mathcal{Q}^{\mathrm{d}}$, in contradiction with classical results on gas mixtures [56, 57, $62,63,64]$.

A mathematical analysis of the structure of these systems of partial differential equations should next be conducted. Such mathematical studies have already concerned the isothermal as well as nonisothermal single species diffuse interface fluids [90, 91, 92]. The situation of nonisothermal Cahn-Hilliard fluids, on the other hand, has not yet been considered, and will be considered in future studies.

\subsection{Entropic considerations}

The macroscopic entropy $\mathcal{S}^{\mathrm{K},(0)}$ obtained from the kinetic model when using higher order Taylor expansion of pair distribution functions may be shown to be in the form

$$
\mathcal{S}^{\mathrm{K},(0)}=\mathcal{S}^{\mathrm{u}}+\sum_{i, j \in \mathfrak{S}} \frac{\varkappa_{i j}}{2 T} \rho_{i} \Delta \rho_{j}
$$

where $\mathcal{S}^{\mathrm{u}}=\mathcal{S}^{(0)}$ is the nonideal entropy obtained in from the kinetic theory (4.16) in the absence of diffuse interface effects. In the situation of constant capillary coefficients, this entropy $\mathcal{S}^{\mathrm{u}}=\mathcal{S}^{(0)}$ has also been shown to be the Gibbsian entropy of the diffuse interface model. We may also transform this entropy (7.8) into

$$
\widetilde{\mathcal{S}}^{\mathrm{K},(0)}=\mathcal{S}^{\mathrm{u}}-\sum_{i, j \in \mathfrak{S}} \frac{\varkappa_{i j}}{2 T} \nabla \rho_{i} \cdot \nabla \rho_{j},
$$

by modifying the entropy flux. 
We thus conclude that the macroscopic entropies $\mathcal{S}^{\mathrm{K},(0)}$ or $\widetilde{\mathcal{S}}^{\mathrm{K},(0)}$ obtained from the kinetic theory by using higher order Taylor expansion of pair distribution functions differ from the Gibbsian entropy $\mathcal{S}^{\mathrm{u}}$ naturally arising from the structure of the governing equations. This Gibbsian entropy $\mathcal{S}^{\mathrm{u}}$ may be seen as a thermodynamic mathematical construction derived from the structure of the system of partial differentail equations. This difference between $\widetilde{\mathcal{S}}^{\mathrm{K},(0)}$ and $\mathcal{S}^{\mathrm{u}}=\mathcal{S}^{(0)}$ may also be interpreted as an indication that there is no $\mathrm{H}$ theorem in general for the generalized Boltzmann equations derived from the BBGKY hierarchy.

A similar situation is that of second order entropies in Enskog expansions, i.e., entropies obtained in Burnett regimes, discussed notably by de Groot and Mazur [59] and Giovangigli [93, 94, 95]. These entropies also differ from the Gibbsian entropies associated with the fluid equations [59].

\subsection{Nonideal fluid thermodynamics and transport}

The derivation of the van der Waals equation of state from the kinetic theory of gas mixtures has used the assumption that the volume ratio is small $\sum_{j \in \mathfrak{S}} n_{j} \mathrm{~b}_{j} \ll 1$. This property then implies that $\left(1-\sum_{j \in \mathfrak{S}} n_{j} \mathrm{~b}_{j}\right)^{-1} \approx 1+\sum_{j \in \mathfrak{S}} n_{j} \mathrm{~b}_{j}$ as in the traditional derivation from from equilibrium statistical mechanics for a single gas $[84,85,86]$. The domain of validity of van der Waals equation of state, however, is much larger than that of the hypotheses made in the derivation and notably includes liquids where the volume ratio $\sum_{j \in \mathfrak{S}} n_{j} \mathrm{~b}_{j}$ is of order unity. Another derivation of the van der Waals equation of state is also possible by directly including factors in the form $\left(1-\sum_{j \in \mathfrak{S}} n_{j} \mathrm{~b}_{j}\right)^{-1}$ in the correlation function $g_{i j}$ as first suggested by Sobrino for single species fluids [34] and used in various work $[40,42]$. This would have the advantage of directly giving factors $\left(1-\sum_{j \in \mathfrak{S}} n_{j} \mathrm{~b}_{j}\right)^{-1}$ in the repulsive part but would also yield such factors in the attractive part of the equation of state and in all capillary terms without further simplifications. This is why we have used the equilibrium correlation functions given by statistical mechanics (4.6) and followed the traditional derivation of van der Waals equation of state from statistical mechanics $[84,85,86]$.

Including triple collisions in the model, taking into account the triplet distributions $f_{i j k}$, would lead to cubic terms in the equation of state. Equations of states like the Redlich-Kwong equation of state [96, 78] could eventually be derived from a kinetic theory including triple collisions. Such equations of state, that are variants of that of van der Waals', and their corresponding thermodynamics [78], yield accurate results over the range of pressure, temperature and mixture states of interest of many phenomena like supercritical combustion [97, 9]. An alternative method could be to modify the collision operators as in Sobrino [34].

The traditional multicomponent diffusion driving forces $\boldsymbol{d}_{i}^{\mathrm{cl}}$ are defined by subtracting temperature gradients from thermodynamic diffusion driving forces $\boldsymbol{d}_{i}^{\text {th }}$ naturally related to chemical potential gradients (4.46). The traditional multicomponent diffusion coefficients are then obtained as detailed in Section 4.5.2 and involve pressure based thermodynamic properties. These traditional expressions may thus only be used when mechanical stability holds and solely the thermodynamic formulation is generally valid. The pressure based species enthalpies and specific volume even explode at mechanical instability limits as detailed in Gaillard et al. [9]. The impact of nonideal thermodynamic forces in multicomponent diffusion has also been investigated in [97, 98]. 


\subsection{Cahn-Hilliard fluids}

We have presented in previous sections the first molecular derivation of Cahn-Hilliard fluids models in the framework of the kinetic theory of dense gas mixtures. This resulting model notably includes generalized van der Waals/Cahn-Hilliard gradient energy, Korteweg tensor, Dunn and Serrin type heat flux, as well as Cahn-Hilliard multicomponent diffusive type fluxes and first order capillary contribution in the heat flux. The capillarity coefficients have also been related to intermolecular forces and the van der Waals equation of state has also been derived. We now address the validity domain of the derivation from the kinetic theory.

The capillary fluid equations have been derived within the kinetic theory of moderately dense gas mixtures essentially assuming that $\sigma^{\star} \ll r^{\star} \ll l_{\mathrm{K}}^{\star} \ll l_{\mathrm{H}}^{\star}$, where $\sigma^{\star}$ is a typical collision diameter or equivalently a typical range of the interaction potentials $\varphi_{i j}, r^{\star}$ a characteristic interparticle distance, $l_{\mathrm{K}}^{\star}$ the mean free path, and $l_{\mathrm{H}}^{\star}$ a hydrodynamic length. We have also assumed that the interaction potentials are hard in order to simplify some of the collision integrals associated with dense gases. We have also assumed that the temperature gradients remain of macroscopic scale as well as that the velocity gradients multiplied by the relative Mach number are small in interphase fronts when estimating the deviations arising from Bogoliubov distributions. However, these capillary equation also apply to liquids where $\sigma^{\star}=r^{\star}=l_{\mathrm{K}}^{\star}$ and are exactly the equations derived from thermodynamics. Therefore, as for the van der Waals equation of state, the domain of validity of the resulting equations is much larger than that of the kinetic theory of moderately dense gases since it also applies to liquids.

We have also used a new Enskog type scaling of the generalized Boltzmann equations. This scaling clarifies the derivation of the macroscopic equations and directly yields the equilibrium Maxwellian distributions. This scaling also yields the Euler capillary fluid equations at zeroth order whereas in previous work for single species fluids the diffuse interface terms were only obtained at first order [15]. In other words, the diffuse interface terms in Euler equations, as well as the nonideal thermodynamic functions, are direct consequences of the perturbed source terms $\mathcal{J}_{i}^{(1)}, i \in \mathfrak{S}$. The importance of diffuse interface Euler fluid equations in conjunction with Hilbert's sixth problem has notably been emphasized by Gorban and Karlin [99, 100] and Slemrod [101].

The derivation from the kinetic theory has been performed by simplifying the capillarity coefficients $\varkappa_{i j}$ so that it is independant of $T$ and from the number densities $n_{i}, i \in \mathfrak{S}$. The temperature dependence of the capillary coefficients could next be investigated by using nonequilibrium correlation functions $g_{i j}$ that may also lead to temperature gradients in the capillary terms. However, there is no clear expression for a nonequilibrium correlation function at interfaces so that it should be chosen carefully [3, 88, 89].

Finally, a key argument in the derivation is the use of a generalized Boltzmann equation with distribution functions are evaluated at different spatial positions.

\section{Conclusion}

We have derived the full Cahn Hilliard fluid equations from the kinetic theory of dense gas mixtures. Only monatomic mixtures of gases have been taken into account and investigating polyatomic gas mixtures in a semi-quantum framework would be of high scientific interest. In the same vein, it would be interesting to investigate reactive source terms treated typically as perturbations. Taking into account triple collisions in the kinetic model would also lead to richer thermodynamic models and state law with third powers 
of concentrations like an approximate Redlich-Kwong state law. The situation of temperature dependent diffuse interface coefficients would also be of high scientific interest and may further lead to extra temperature gradients in the capillary terms. Boundary conditions at solid walls and three phase lines could also be investigated with kinetic methods by taking into account interaction potentials with crystal atoms as well as collisions with phonons. Numerical simulations would also be an outstanding tool for comparing kinetic models and their corresponding macroscopic diffuse interface equations. Last but not least, the mathematical structure of the resulting systems of partial differential equations should be investigated in depth.

\section{A Entropy production in fluid models}

We derive in this section the rate of entropy production (2.12) from Gibbs relation (2.8) and the fluid governing equations (2.9)-(2.11). The species conservation equations are first written in the form

$$
\partial_{t} \rho_{i}+\boldsymbol{v} \cdot \nabla \rho_{i}=-\rho_{i} \boldsymbol{\nabla} \cdot \boldsymbol{v}-\boldsymbol{\nabla} \cdot \overline{\mathcal{F}}_{i}, \quad i \in \mathfrak{S},
$$

and we also have the differential identity

$$
\left(\partial_{t}+\boldsymbol{v} \cdot \boldsymbol{\nabla}\right) \boldsymbol{\nabla} \rho_{i}=\boldsymbol{\nabla}\left(\partial_{t} \rho_{i}+\boldsymbol{v} \cdot \boldsymbol{\nabla} \rho_{i}\right)-(\boldsymbol{\nabla} \boldsymbol{v})^{t} \cdot \boldsymbol{\nabla} \rho_{i}, \quad i \in \mathfrak{S} .
$$

Multiplying the momentum conservation equation (2.10) by the velocity vector $\boldsymbol{v}$ and subtracting the result from the total energy conservation equation (2.11) also yields the balance equation for internal energy $\mathcal{E}$ in the form

$$
\partial_{t} \mathcal{E}+\boldsymbol{v} \cdot \nabla \mathcal{E}=-\mathcal{E} \nabla \cdot \boldsymbol{v}-\nabla \cdot \mathcal{Q}-\mathcal{P}: \nabla v
$$

Using Gibbs relation (2.8), we further obtain that

$$
T\left(\partial_{t} \mathcal{S}+\boldsymbol{v} \cdot \nabla \mathcal{S}\right)=\partial_{t} \mathcal{E}+\boldsymbol{v} \cdot \nabla \mathcal{E}-\sum_{i \in \mathfrak{S}} \bar{g}_{i}\left(\partial_{t} \rho_{i}+\boldsymbol{v} \cdot \nabla \rho_{i}\right)-\sum_{i \in \mathfrak{S}} \bar{\gamma}_{i} \cdot\left(\partial_{t}+\boldsymbol{v} \cdot \boldsymbol{\nabla}\right) \boldsymbol{\nabla} \rho_{i},
$$

and combining this with relations (A.1)-(A.3) yields

$$
\begin{aligned}
\partial_{t} \mathcal{S}+\boldsymbol{v} \cdot \boldsymbol{\nabla} \mathcal{S}= & -\frac{1}{T}(\mathcal{E} \boldsymbol{\nabla} \cdot \boldsymbol{v}+\boldsymbol{\nabla} \cdot \mathcal{Q}+\mathcal{P}: \boldsymbol{\nabla} \boldsymbol{v}) \\
& +\sum_{i \in \mathfrak{S}} \frac{\bar{g}_{i}}{T}\left(\rho_{i} \boldsymbol{\nabla} \cdot \boldsymbol{v}+\boldsymbol{\nabla} \cdot \overline{\mathcal{F}}_{i}\right) \\
& +\sum_{i \in \mathfrak{S}} \frac{\bar{\gamma}_{i}}{T} \cdot\left(\boldsymbol{\nabla}\left(\rho_{i} \boldsymbol{\nabla} \cdot \boldsymbol{v}+\boldsymbol{\nabla} \cdot \overline{\mathcal{F}}_{i}\right)+(\boldsymbol{\nabla} \boldsymbol{v})^{t} \boldsymbol{\nabla} \rho_{i}\right) .
\end{aligned}
$$

The aim is now to decompose the right hand side of (A.4) into an entropy flux and a nonnegative production term. A key argument is that derivatives may take arbitrary values so that only products of derivatives of the same order may have a sign. We may also regroup all terms proportional to $\boldsymbol{\nabla} \cdot \boldsymbol{v}$ by using $(\mathcal{G}-\mathcal{E}) / T=p / T-\mathcal{S}$.

Since the divergence of the total heat flux $\boldsymbol{\nabla} \cdot \mathcal{Q}$ involves second order derivatives of temperature, it must be integrated by part. Similarly, the terms $\boldsymbol{\nabla}\left(\boldsymbol{\nabla} \cdot \overline{\mathcal{F}}_{i}\right)$ involves fifth 
order derivatives with Cahn-Hilliard type fluxes - third order with standard diffusionand must also be integrated by parts. The terms $\bar{\gamma}_{i} \cdot(\boldsymbol{\nabla v})^{t} \cdot \boldsymbol{\nabla} \rho_{i}$ may further be regrouped with the velocity gradient terms and these operations yield

$$
\begin{aligned}
\partial_{t} \mathcal{S}+\boldsymbol{\nabla} \cdot(\boldsymbol{v} \mathcal{S})+ & \boldsymbol{\nabla} \cdot\left(\frac{\mathcal{Q}}{T}-\sum_{i \in \mathfrak{S}} \frac{\bar{\gamma}_{i}}{T}\left(\rho_{i} \boldsymbol{\nabla} \cdot \boldsymbol{v}+\boldsymbol{\nabla} \cdot \overline{\mathcal{F}}_{i}\right)\right) \\
= & -\frac{1}{T}\left(\mathcal{P}-p \boldsymbol{I}-\sum_{i \in \mathfrak{S}} \nabla \rho_{i} \otimes \overline{\boldsymbol{\gamma}}_{i}\right): \boldsymbol{\nabla} \boldsymbol{v}-\mathcal{Q} \cdot \frac{\boldsymbol{\nabla} T}{T^{2}} \\
& +\sum_{i \in \mathfrak{S}}\left(\frac{\bar{g}_{i}}{T}-\nabla \cdot \frac{\bar{\gamma}_{i}}{T}\right) \nabla \cdot \overline{\mathcal{F}}_{i}-\sum_{i \in \mathfrak{S}} \boldsymbol{\nabla} \cdot \frac{\bar{\gamma}_{i}}{T} \rho_{i} \boldsymbol{\nabla} \cdot \boldsymbol{v} .
\end{aligned}
$$

The derivatives $\boldsymbol{\nabla} \cdot\left(\bar{\gamma}_{i} / T\right)$ appearing in the two last sums on the right hand side are then developed and the resulting terms split between temperature and velocity gradients, as for a single species fluid [15]. The diffusive fluxes are also integrated by parts and these operations yield

$$
\begin{aligned}
\partial_{t} \mathcal{S}+\boldsymbol{\nabla} \cdot(\boldsymbol{v} \mathcal{S})+ & \cdot\left(\frac{\mathcal{Q}}{T}-\sum_{i \in \mathfrak{S}} \frac{\overline{\boldsymbol{\gamma}}_{i}}{T}\left(\rho_{i} \boldsymbol{\nabla} \cdot \boldsymbol{v}+\boldsymbol{\nabla} \cdot \overline{\mathcal{F}}_{i}\right)-\sum_{i \in \mathfrak{S}}\left(\frac{\bar{g}_{i}}{T}-\frac{\boldsymbol{\nabla} \cdot \overline{\boldsymbol{\gamma}}_{i}}{T}\right) \overline{\mathcal{F}}_{i}\right) \\
= & -\frac{1}{T}\left(\mathcal{P}-p \boldsymbol{I}-\sum_{i \in \mathfrak{S}}\left(\boldsymbol{\nabla} \rho_{i} \otimes \overline{\boldsymbol{\gamma}}_{i}-\rho_{i} \boldsymbol{\nabla} \cdot \overline{\boldsymbol{\gamma}}_{i} \boldsymbol{I}\right)\right): \boldsymbol{\nabla} \boldsymbol{v} \\
& -\left(\mathcal{Q}-\sum_{i \in \mathfrak{S}} \bar{\gamma}_{i}\left(\rho_{i} \boldsymbol{\nabla} \cdot \boldsymbol{v}+\boldsymbol{\nabla} \cdot \overline{\mathcal{F}}_{i}\right)\right) \cdot \boldsymbol{\nabla}\left(\frac{-1}{T}\right) \\
& -\sum_{i \in \mathfrak{S}} \boldsymbol{\nabla}\left(\frac{\bar{g}_{i}}{T}-\frac{\boldsymbol{\nabla} \cdot \bar{\gamma}_{i}}{T}\right) \cdot \overline{\mathcal{F}}_{i}
\end{aligned}
$$

We may finally develop the terms $\nabla \frac{\nabla \cdot \bar{\gamma}_{i}}{T}$ in the diffusion driving forces to finally obtain that

$$
\begin{aligned}
\partial_{t} \mathcal{S}+\boldsymbol{\nabla} \cdot(\boldsymbol{v} \mathcal{S})+ & \boldsymbol{\nabla} \cdot\left(\frac{\mathcal{Q}}{T}-\sum_{i \in \mathfrak{S}} \frac{\bar{\gamma}_{i}}{T}\left(\rho_{i} \boldsymbol{\nabla} \cdot \boldsymbol{v}+\boldsymbol{\nabla} \cdot \overline{\mathcal{F}}_{i}\right)-\sum_{i \in \mathfrak{S}}\left(\frac{\bar{g}_{i}}{T}-\frac{\boldsymbol{\nabla} \cdot \bar{\gamma}_{i}}{T}\right) \overline{\mathcal{F}}_{i}\right) \\
= & -\frac{1}{T}\left(\mathcal{P}-p \boldsymbol{I}-\sum_{i \in \mathfrak{S}}\left(\boldsymbol{\nabla} \rho_{i} \otimes \overline{\boldsymbol{\gamma}}_{i}-\rho_{i} \boldsymbol{\nabla} \cdot \overline{\boldsymbol{\gamma}}_{i} \boldsymbol{I}\right)\right): \boldsymbol{\nabla} \boldsymbol{v} \\
& -\left(\mathcal{Q}-\sum_{i \in \mathfrak{S}} \bar{\gamma}_{i}\left(\rho_{i} \boldsymbol{\nabla} \cdot \boldsymbol{v}+\boldsymbol{\nabla} \cdot \overline{\mathcal{F}}_{i}\right)+\sum_{i \in \mathfrak{S}} \boldsymbol{\nabla} \cdot \overline{\boldsymbol{\gamma}}_{i} \overline{\mathcal{F}}_{i}\right) \cdot \boldsymbol{\nabla}\left(\frac{-1}{T}\right) \\
& -\sum_{i \in \mathfrak{S}}\left(\boldsymbol{\nabla} \frac{\bar{g}_{i}}{T}-\frac{\boldsymbol{\nabla}\left(\boldsymbol{\nabla} \cdot \overline{\boldsymbol{\gamma}}_{i}\right)}{T}\right) \cdot \overline{\mathcal{F}}_{i} .
\end{aligned}
$$

This is the balance of entropy used in Section 2.2 involving notably the force terms $\boldsymbol{\nabla}\left(\bar{g}_{i} / T\right)-\boldsymbol{\nabla} \nabla \cdot \bar{\gamma}_{i} / T$, a Korteweg type tensor and a Dunn and Serrin type heat flux.

Another variant is then to integrate by part the diffusion terms in (A.5) to get that

$$
\begin{aligned}
\partial_{t} \mathcal{S}+\boldsymbol{\nabla} \cdot(\boldsymbol{v} \mathcal{S})+ & \boldsymbol{\nabla} \cdot\left(\frac{\mathcal{Q}}{T}-\sum_{i \in \mathfrak{S}} \frac{\bar{\gamma}_{i}}{T}\left(\rho_{i} \boldsymbol{\nabla} \cdot \boldsymbol{v}+\boldsymbol{\nabla} \cdot \overline{\mathcal{F}}_{i}\right)-\sum_{i \in \mathfrak{S}}\left(\frac{\bar{g}_{i}}{T}-\nabla \cdot \frac{\bar{\gamma}_{i}}{T}\right) \overline{\mathcal{F}}_{i}\right) \\
= & -\frac{1}{T}\left(\mathcal{P}-p \boldsymbol{I}-\sum_{i \in \mathfrak{S}}\left(\nabla \rho_{i} \otimes \overline{\boldsymbol{\gamma}}_{i}-\rho_{i} \boldsymbol{\nabla} \cdot \bar{\gamma}_{i} \boldsymbol{I}\right)\right): \boldsymbol{\nabla} \boldsymbol{v} \\
& -\left(\mathcal{Q}-\sum_{i \in \mathfrak{S}} \rho_{i} \bar{\gamma}_{i} \boldsymbol{\nabla} \cdot \boldsymbol{v}\right) \cdot \boldsymbol{\nabla}\left(\frac{-1}{T}\right)-\sum_{i \in \mathfrak{S}} \boldsymbol{\nabla}\left(\frac{\bar{g}_{i}}{T}-\boldsymbol{\nabla} \cdot \overline{\boldsymbol{\gamma}}_{i}\right) \cdot \overline{\mathcal{F}}_{i} .
\end{aligned}
$$


Finally, a fourth variant is obtained by developing the derivatives $\nabla\left(\bar{g}_{i} / T\right)$ in the diffusion driving forces of (A.7) and the result reads

$$
\begin{aligned}
\partial_{t} \mathcal{S}+\boldsymbol{\nabla} \cdot(\boldsymbol{v} \mathcal{S})+ & \boldsymbol{\nabla} \cdot\left(\frac{\mathcal{Q}}{T}-\sum_{i \in \mathfrak{S}} \frac{\bar{\gamma}_{i}}{T}\left(\rho_{i} \boldsymbol{\nabla} \cdot \boldsymbol{v}+\nabla \cdot \overline{\mathcal{F}}_{i}\right)-\sum_{i \in \mathfrak{S}}\left(\frac{\bar{g}_{i}}{T}-\frac{\nabla \cdot \bar{\gamma}_{i}}{T}\right) \overline{\mathcal{F}}_{i}\right) \\
= & -\frac{1}{T}\left(\mathcal{P}-p \boldsymbol{I}-\sum_{i \in \mathfrak{S}}\left(\nabla \rho_{i} \otimes \bar{\gamma}_{i}-\rho_{i} \boldsymbol{\nabla} \cdot \bar{\gamma}_{i} \boldsymbol{I}\right)\right): \nabla \boldsymbol{v} \\
& -\left(\mathcal{Q}-\sum_{i \in \mathfrak{S}} \bar{\gamma}_{i}\left(\rho_{i} \boldsymbol{\nabla} \cdot \boldsymbol{v}+\nabla \cdot \overline{\mathcal{F}}_{i}\right)-\sum_{i \in \mathfrak{S}}\left(\bar{g}_{i}-\nabla \cdot \bar{\gamma}_{i}\right) \overline{\mathcal{F}}_{i}\right) \cdot \boldsymbol{\nabla}\left(\frac{-1}{T}\right) \\
& -\sum_{i \in \mathfrak{S}} \frac{1}{T}\left(\nabla \bar{g}_{i}-\nabla\left(\nabla \cdot \bar{\gamma}_{i}\right)\right) \cdot \overline{\mathcal{F}}_{i} .
\end{aligned}
$$

The four expressions (A.6) (A.7), (A.8) and (A.9) are discussed in Section 7.1 and also reveals the ambiguity of rational thermodynamics.

\section{B Cahn-Hilliard models with mass fraction gradients}

The general form for free energies $\mathcal{A}$ in Cahn-Hilliard models has been written in Section 2.1 by using partial density gradients (2.1). Such energies have notably been used by Cahn [16], Rowlinson and Widom [3], Verschueren [20], Liu et al. [23], and Heida et al.[21] and reduce to the traditional van der Waals energy for a single species fluid.

However, historically, gradient energies in terms of mole fraction gradients have first been considered by Cahn and Hilliard. We thus briefly address such a situation in this section, easily derived from the general case of density gradients. More specifically, we consider energies written in terms of mass fraction gradients as Anderson et al. [2], that are more convenient than mole fractions for multicomponent mixtures and mass based diffusion fluxes. Note still that mole and mass also coincide for isomass mixtures. We have to establish in particular that there are not anymore second order derivatives in the generalized Korteweg Pressure tensor as traditionally established with such energies [2].

We consider thus an energy written in the form

$$
\mathcal{A}=\mathcal{A}^{\mathrm{u}}+\sum_{i, j \in \mathfrak{S}} \frac{1}{2} \bar{\chi}_{i j} \nabla \mathrm{y}_{i} \cdot \nabla \mathrm{y}_{j}
$$

where $\mathrm{y}_{i}=\rho_{i} / \rho$ denotes the mass fraction of the $i$ th species and $\rho=\sum_{i \in \mathfrak{S}} \rho_{i}$ the mixture mass density. Noting then that

$$
\nabla \mathrm{y}_{i}=\frac{1}{\rho}\left(\nabla \rho_{i}-\mathrm{y}_{i} \boldsymbol{\nabla} \rho\right)=\frac{1}{\rho} \sum_{k \in \mathfrak{S}}\left(\delta_{i k}-\mathrm{y}_{i}\right) \nabla \rho_{k},
$$

we may rewrite the energy (B.1) in the general form (2.1), provided the mass-based capillary coefficients $\bar{\varkappa}_{k l}$ are in the special form

$$
\bar{\varkappa}_{k l}=\sum_{i, j \in \mathfrak{S}} \bar{\chi}_{i j} \frac{1}{\rho^{2}}\left(\delta_{i k}-\mathrm{y}_{i}\right)\left(\delta_{j l}-\mathrm{y}_{j}\right), \quad k, l \in \mathfrak{S} .
$$


We may now evaluate the Korteweg tensor $\mathcal{P}^{\mathrm{KO}}$ by first noting that

$$
\begin{aligned}
-\sum_{k, l \in \mathfrak{S}} \rho_{k} \boldsymbol{\nabla} \cdot\left(\bar{\varkappa}_{k l} \nabla \rho_{l}\right) & =-\sum_{i, j, k, l \in \mathfrak{S}} \rho_{k} \nabla \cdot\left(\bar{\chi}_{i j} \frac{1}{\rho^{2}}\left(\delta_{i k}-\mathrm{y}_{i}\right)\left(\delta_{j l}-\mathrm{y}_{j}\right) \nabla \rho_{l}\right) \\
& =-\sum_{i, j, k \in \mathfrak{S}} \rho_{k} \nabla \cdot\left(\bar{\chi}_{i j} \frac{1}{\rho}\left(\delta_{i k}-\mathrm{y}_{i}\right) \nabla \mathrm{y}_{j}\right) \\
& =\sum_{i, j \in \mathfrak{S}} \bar{\chi}_{i j} \nabla \mathrm{y}_{i} \cdot \nabla \mathrm{y}_{j},
\end{aligned}
$$

where use has been made of $\sum_{k \in \mathfrak{S}} \rho_{k}\left(\delta_{i k}-\mathrm{y}_{i}\right)=0$ after expanding the divergence term. On the other hand, a direct calculations yields

$$
\frac{1}{2} \sum_{k, l \in \mathfrak{S}}\left(\sum_{m \in \mathfrak{S}} \rho_{m} \partial_{\rho_{m}} \bar{\varkappa}_{k l}-\bar{\varkappa}_{k l}\right) \nabla \rho_{k} \cdot \nabla \rho_{l}=\frac{1}{2} \sum_{i, j \in \mathfrak{S}}\left(\sum_{m \in \mathfrak{S}} \rho_{m} \partial_{\rho_{m}} \bar{\chi}_{i j}-3 \bar{\chi}_{i j}\right) \nabla \mathrm{y}_{i} \cdot \nabla \mathrm{y}_{j},
$$

in such a way that

$$
\mathcal{P}^{\mathrm{KO}}=\frac{1}{2} \sum_{i, j \in \mathfrak{S}}\left(\sum_{k \in \mathfrak{S}} \rho_{k} \partial_{\rho_{k}} \bar{\chi}_{i j}-\bar{\chi}_{i j}\right) \nabla \mathrm{y}_{i} \cdot \nabla \mathrm{y}_{j} \boldsymbol{I}+\sum_{i, j \in \mathfrak{S}} \bar{\chi}_{i j} \nabla \mathrm{y}_{i} \otimes \nabla \mathrm{y}_{j}
$$

In particular, there are not anymore second order derivatives in the pressure tensor $\mathcal{P}^{\mathrm{KO}}$ as traditionally obtained [2] when using mass or mole fractions gradients in the free energy as in (B.1).

Finally, a direct calculation yields that the generalized Dunn and Serrin heat flux vanishes $\mathcal{Q}^{\mathrm{DS}}=0$ and that we also have

$$
\mathcal{Q}^{\mathrm{CD}}=-\sum_{i, j \in \mathfrak{S}} \nabla \cdot\left(\frac{1}{\rho} \bar{\chi}_{i j} \nabla \mathrm{y}_{j}\right) \mathcal{F}_{i}+\sum_{i, j \in \mathfrak{S}} \frac{1}{\rho} \bar{\chi}_{i j} \nabla \mathrm{y}_{j} \nabla \cdot \mathcal{F}_{i} .
$$

\section{Macroscopic equations from the kinetic theory}

\section{C.1 Derivation from the hierarchy}

Multiplying by $m_{i}$ the Boltzmann equation of the $i$ th species (3.1), integrating with respect to $\boldsymbol{c}_{i}$, and decomposing $\boldsymbol{c}_{i}=\boldsymbol{v}+\boldsymbol{c}_{i}-\boldsymbol{v}$, it is obtained that

$$
\partial_{t} \rho_{i}+\nabla \cdot\left(\rho_{i} \boldsymbol{v}\right)+\nabla \cdot \overline{\mathcal{F}}_{i}=0
$$

where the diffusive mass flux of the $i$ th species $\overline{\mathcal{F}}_{i}$ is defined by

$$
\overline{\mathcal{F}}_{i}(\mathbf{r}, t)=\int m_{i}\left(\boldsymbol{c}_{i}-\boldsymbol{v}\right) f_{i}\left(\mathbf{r}, \boldsymbol{c}_{i}, t\right) \mathrm{d} \boldsymbol{c}_{i} .
$$

Multiplying by $m_{i} \boldsymbol{c}_{i}$ the Boltzmann equation (3.1), integrating with respect to $\boldsymbol{c}_{i}$, summing over the species $i \in \mathfrak{S}$, and using the definition of $\boldsymbol{v}$ then yields

$$
\partial_{t}(\rho \boldsymbol{v})+\nabla \cdot(\rho \boldsymbol{v} \otimes \boldsymbol{v})+\nabla \cdot \mathcal{P}^{\mathrm{K}}+\mathcal{R}^{\boldsymbol{v}}=0,
$$

where $\mathcal{P}^{\mathrm{K}}$ is the traditional kinetic part of the pressure tensor

$$
\mathcal{P}^{\mathrm{K}}(\mathbf{r}, t)=\sum_{i \in \mathfrak{S}} \int m_{i}\left(\boldsymbol{c}_{i}-\boldsymbol{v}\right) \otimes\left(\boldsymbol{c}_{i}-\boldsymbol{v}\right) f_{i}\left(\mathbf{r}, \boldsymbol{c}_{i}, t\right) \mathrm{d} \boldsymbol{c}_{i},
$$


and the term $\mathcal{R}^{v}$ reads

$$
\mathcal{R}^{\boldsymbol{v}}(\mathbf{r}, t)=-\sum_{i, j \in \mathfrak{S}} \int m_{i} \boldsymbol{c}_{i} \theta_{i j} f_{i j}\left(\mathbf{r}, \boldsymbol{c}_{i}, \mathbf{r}+\mathbf{r}_{i j}, \boldsymbol{c}_{j}, t\right) \mathrm{d} \boldsymbol{c}_{i} \mathrm{~d} \mathbf{r}_{i j} \mathrm{~d} \boldsymbol{c}_{j}
$$

with $\mathbf{r}_{i j}=\mathbf{r}_{j}-\mathbf{r}_{i}$. Using the expression of $\theta_{i j}$, integrating by parts with respect to $\boldsymbol{c}_{i}$ and $\boldsymbol{c}_{j}$, using the definition of $n_{i j}$ and $\partial_{\mathbf{r}_{i}} \varphi_{i j}=-\varphi_{i j}^{\prime}\left(r_{i j}\right) \mathbf{r}_{i j} / r_{i j}$, it is obtained that

$$
\mathcal{R}^{\boldsymbol{v}}(\mathbf{r}, t)=-\sum_{i, j \in \mathfrak{S}} \int \frac{\varphi_{i j}^{\prime}\left(r_{i j}\right)}{r_{i j}} \mathbf{r}_{i j} n_{i j}\left(\mathbf{r}, \mathbf{r}+\mathbf{r}_{i j}, t\right) \mathrm{d} \mathbf{r}_{i j}
$$

We may now use that $n_{i j}\left(\mathbf{r}, \mathbf{r}+\mathbf{r}_{i j}, t\right)=n_{j i}\left(\mathbf{r}+\mathbf{r}_{i j}, \mathbf{r}, t\right)$, letting $\mathbf{r}_{j i}=-\mathbf{r}_{i j}$, and exchanging $i$ and $j$ in the resulting sum, we get

$$
\mathcal{R}^{v}(\mathbf{r}, t)=\sum_{i, j \in \mathfrak{S}} \int \frac{\varphi_{i j}^{\prime}\left(r_{i j}\right)}{r_{i j}} \mathbf{r}_{i j} n_{i j}\left(\mathbf{r}-\mathbf{r}_{i j}, \mathbf{r}, t\right) \mathrm{d} \mathbf{r}_{i j}
$$

and thus

$$
\mathcal{R}^{v}(\mathbf{r}, t)=-\sum_{i, j \in \mathfrak{S}} \frac{1}{2} \int \frac{\varphi_{i j}^{\prime}\left(r_{i j}\right)}{r_{i j}} \mathbf{r}_{i j}\left(n_{i j}\left(\mathbf{r}, \mathbf{r}+\mathbf{r}_{i j}, t\right)-n_{i j}\left(\mathbf{r}-\mathbf{r}_{i j}, \mathbf{r}, t\right)\right) \mathrm{d} \mathbf{r}_{i j}
$$

Letting then $\bar{n}_{i j}\left(\mathbf{r}, \mathbf{r}_{i j}, \alpha\right)=n_{i j}\left(\mathbf{r}-(1-\alpha) \mathbf{r}_{i j}, \mathbf{r}+\alpha \mathbf{r}_{i j}, t\right)$ we have

$$
\bar{n}_{i j}(1)-\bar{n}_{i j}(0)=n_{i j}\left(\mathbf{r}, \mathbf{r}+\mathbf{r}_{i j}, t\right)-n_{i j}\left(\mathbf{r}-\mathbf{r}_{i j}, \mathbf{r}, t\right)=\int_{0}^{1} \partial_{\alpha} \bar{n}_{i j}(\alpha) \mathrm{d} \alpha .
$$

Since $\partial_{\alpha} \bar{n}_{i j}(\alpha)=\partial_{\mathbf{r}} \bar{n}_{i j} \cdot \mathbf{r}_{i j}$ we finally obtain that $\boldsymbol{\mathcal { R }}^{\boldsymbol{v}}(\mathbf{r}, t)=\boldsymbol{\nabla} \cdot \mathcal{P}^{\mathrm{P}}(\mathbf{r}, t)$ where

$$
\mathcal{P}^{\mathrm{P}}(\mathbf{r}, t)=-\sum_{i, j \in \mathfrak{S}} \frac{1}{2} \int \frac{\varphi_{i j}^{\prime}\left(r_{i j}\right)}{r_{i j}} \mathbf{r}_{i j} \otimes \mathbf{r}_{i j} n_{i j}\left(\mathbf{r}-(1-\alpha) \mathbf{r}_{i j}, \mathbf{r}+\alpha \mathbf{r}_{i j}, t\right) \mathrm{d} \alpha \mathrm{d} \mathbf{r}_{i j},
$$

and the scalar $\alpha$ must be integrated over $(0,1)$. The tensor $\mathcal{P}^{\mathrm{K}}$ corresponds to the transfer of momentum due to the flow of particles and $\mathcal{P}^{\mathrm{P}}$ to the transfer of momentum between the species particles due to intermolecular forces [56, 57].

The kinetic part of the internal energy conservation equation is first obtained by multiplying Boltzmann equation (3.1) by $\frac{1}{2} m_{i}\left|\boldsymbol{c}_{i}-\boldsymbol{v}\right|^{2}$, integrating with respect to $\boldsymbol{c}_{i}$, and summing over the species $i \in \mathfrak{S}$. Using the definitions of $\mathcal{E}^{\mathrm{K}}$ and $\mathcal{P}^{\mathrm{K}}$ yields after some algebra

$$
\partial_{t} \mathcal{E}^{\mathrm{K}}+\boldsymbol{\nabla} \cdot\left(\boldsymbol{v} \mathcal{E}^{\mathrm{K}}\right)+\boldsymbol{\nabla} \cdot \mathcal{Q}^{\mathrm{K}}+\mathcal{R}_{1}^{\mathcal{E}}=-\mathcal{P}^{\mathrm{K}}: \boldsymbol{\nabla} \boldsymbol{v}
$$

where

$$
\begin{gathered}
\mathcal{Q}^{\mathrm{K}}\left(\mathbf{r}_{i}, t\right)=\sum_{i \in \mathfrak{S}} \int \frac{1}{2} m_{i}\left|\boldsymbol{c}_{i}-\boldsymbol{v}\right|^{2}\left(\boldsymbol{c}_{i}-\boldsymbol{v}\right) f_{i}\left(\mathbf{r}, \boldsymbol{c}_{i}, t\right) \mathrm{d} \boldsymbol{c}_{i}, \\
\boldsymbol{\mathcal { R }}_{1}^{\mathcal{E}}=-\sum_{i, j \in \mathfrak{S}} \int \frac{1}{2} m_{i}\left|\boldsymbol{c}_{i}-\boldsymbol{v}\right|^{2} \theta_{i j} f_{i j}\left(\mathbf{r}, \boldsymbol{c}_{i}, \mathbf{r}+\mathbf{r}_{i j}, \boldsymbol{c}_{j}, t\right) \mathrm{d} \boldsymbol{c}_{i} \mathrm{~d} \mathbf{r}_{i j} \mathrm{~d} \boldsymbol{c}_{j} .
\end{gathered}
$$

Using the definition of $\theta_{i j}, \partial_{\mathbf{r}_{i}} \varphi_{i j}=-\varphi_{i j}^{\prime}\left(r_{i j}\right) \mathbf{r}_{i j} / r_{i j}$, integrating by parts with respect to $\boldsymbol{c}_{i}$ and $\boldsymbol{c}_{j}$, we next obtain that

$$
\boldsymbol{\mathcal { R }}_{1}^{\mathcal{E}}=-\sum_{i, j \in \mathfrak{S}} \int\left(\boldsymbol{c}_{i}-\boldsymbol{v}\right) \cdot \mathbf{r}_{i j} \frac{\varphi_{i j}^{\prime}\left(r_{i j}\right)}{r_{i j}} f_{i j}\left(\mathbf{r}, \boldsymbol{c}_{i}, \mathbf{r}+\mathbf{r}_{i j}, \boldsymbol{c}_{j},, t\right) \mathrm{d} \boldsymbol{c}_{i} \mathrm{~d} \mathbf{r}_{i j} \mathrm{~d} \boldsymbol{c}_{j} .
$$


The governing equation for the potential part $\mathcal{E}^{\mathrm{P}}$ of the internal energy may then conveniently be obtained from the second equation of the BBGKY hierarchy. Multiplying the two-particle distribution function equations (3.2) by the potential energy $\frac{1}{2} \varphi_{i j}\left(r_{i j}\right)$ integrating with respect to $\boldsymbol{c}_{i}, \boldsymbol{c}_{j}$, and $\mathbf{r}_{i j}$, and summing with respect to $i, j \in \mathfrak{S}$, the resulting equation is in the form

$$
\partial_{t} \mathcal{E}^{\mathrm{P}}+\boldsymbol{\nabla} \cdot\left(\boldsymbol{v} \mathcal{E}^{\mathrm{P}}\right)+\boldsymbol{\nabla} \cdot \mathcal{Q}_{1}^{\mathrm{P}}+\mathcal{R}_{2}^{\mathcal{E}}=0,
$$

where

$$
\begin{gathered}
\mathcal{Q}_{1}^{\mathrm{P}}(\mathbf{r}, t)=\sum_{i, j \in \mathfrak{S}} \int \frac{1}{2} \varphi_{i j}\left(r_{i j}\right)\left(\boldsymbol{c}_{i}-\boldsymbol{v}\right) f_{i j}\left(\mathbf{r}, \boldsymbol{c}_{i}, \mathbf{r}_{j}, \boldsymbol{c}_{j}, t\right) \mathrm{d} \boldsymbol{c}_{i} \mathrm{~d} \mathbf{r}_{j} \mathrm{~d} \boldsymbol{c}_{j}, \\
\mathcal{R}_{2}^{\mathcal{E}}=-\sum_{i, j \in \mathfrak{S}} \frac{1}{2} \int\left(\boldsymbol{c}_{j}-\boldsymbol{c}_{i}\right) \cdot \mathbf{r}_{i j} \frac{\varphi_{i j}^{\prime}\left(r_{i j}\right)}{r_{i j}} f_{i j}\left(\mathbf{r}, \boldsymbol{c}_{i}, \mathbf{r}+\mathbf{r}_{i j}, \boldsymbol{c}_{j}, t\right) \mathrm{d} \boldsymbol{c}_{i} \mathrm{~d} \mathbf{r}_{i j} \mathrm{~d} \boldsymbol{c}_{j} .
\end{gathered}
$$

Adding both equations (C.5) and (C.7) for the conservation of $\mathcal{E}^{\mathrm{K}}$ and $\mathcal{E}^{\mathrm{P}}$ then yields

$$
\partial_{t} \mathcal{E}+\boldsymbol{\nabla} \cdot(\mathcal{E} \boldsymbol{v})+\boldsymbol{\nabla} \cdot\left(\mathcal{Q}^{\mathrm{K}}+\mathcal{Q}_{1}^{\mathrm{P}}\right)+\mathcal{R}_{3}^{\mathcal{E}}=-\mathcal{P}^{\mathrm{K}}: \boldsymbol{\nabla} \boldsymbol{v},
$$

where $\mathcal{R}_{3}^{\mathcal{E}}=\mathcal{R}_{1}^{\mathcal{E}}+\boldsymbol{\mathcal { R }}_{2}^{\mathcal{E}}$ reads

$$
\boldsymbol{\mathcal { R }}_{3}^{\mathcal{E}}=-\sum_{i, j \in \mathfrak{S}} \frac{1}{2} \int\left(\boldsymbol{c}_{i}-\boldsymbol{v}+\boldsymbol{c}_{j}-\boldsymbol{v}\right) \cdot \mathbf{r}_{i j} \frac{\varphi_{i j}^{\prime}\left(r_{i j}\right)}{r_{i j}} f_{i j}\left(\mathbf{r}, \boldsymbol{c}_{i}, \mathbf{r}+\mathbf{r}_{i j}, \boldsymbol{c}_{j}, t\right) \mathrm{d} \boldsymbol{c}_{i} \mathrm{~d} \mathbf{r}_{i j} \mathrm{~d} \boldsymbol{c}_{j} .
$$

In order to transform $\mathcal{R}_{3}^{\mathcal{E}}$ we may proceed as for the momentum equation. More specifically, using

$$
f_{i j}\left(\mathbf{r}, \boldsymbol{c}_{i}, \mathbf{r}+\mathbf{r}_{i j}, \boldsymbol{c}_{j}, t\right)=f_{j i}\left(\mathbf{r}+\mathbf{r}_{i j}, \boldsymbol{c}_{j}, \mathbf{r}, \boldsymbol{c}_{i}, t\right),
$$

letting $\mathbf{r}_{j i}=-\mathbf{r}_{i j}$, and exchanging $i$ and $j$ in the sum, we obtain that

$$
\mathcal{R}_{3}^{\mathcal{E}}=\sum_{i, j \in \mathfrak{S}} \frac{1}{2} \int\left(\boldsymbol{c}_{i}-\boldsymbol{v}+\boldsymbol{c}_{j}-\boldsymbol{v}\right) \cdot \mathbf{r}_{i j} \frac{\varphi_{i j}^{\prime}\left(r_{i j}\right)}{r_{i j}} f_{i j}\left(\mathbf{r}-\mathbf{r}_{i j}, \boldsymbol{c}_{i}, \mathbf{r}, \boldsymbol{c}_{j}, t\right) \mathrm{d} \boldsymbol{c}_{i} \mathrm{~d} \mathbf{r}_{i j} \mathrm{~d} \boldsymbol{c}_{j},
$$

so that

$$
\boldsymbol{\mathcal { R }}_{3}^{\mathcal{E}}=-\sum_{i, j \in \mathfrak{S}} \frac{1}{4} \int\left(\boldsymbol{c}_{i}-\boldsymbol{v}+\boldsymbol{c}_{j}-\boldsymbol{v}\right) \cdot \mathbf{r}_{i j} \frac{\varphi_{i j}^{\prime}\left(r_{i j}\right)}{r_{i j}}\left[f_{i j}\right] \mathrm{d} \boldsymbol{c}_{i} \mathrm{~d} \mathbf{r}_{i j} \mathrm{~d} \boldsymbol{c}_{j}
$$

with

$$
\left[f_{i j}\right]=f_{i j}\left(\mathbf{r}, \boldsymbol{c}_{i}, \mathbf{r}+\mathbf{r}_{i j}, \boldsymbol{c}_{j}, t\right)-f_{i j}\left(\mathbf{r}-\mathbf{r}_{i j}, \boldsymbol{c}_{i}, \mathbf{r}, \boldsymbol{c}_{j}, t\right) .
$$

Letting then $\bar{f}_{i j}(\alpha)=f_{i j}\left(\mathbf{r}-(1-\alpha), \boldsymbol{c}_{i}, \mathbf{r}+\alpha \mathbf{r}_{i j}, \boldsymbol{c}_{j}, t\right)$ we have

$$
\left[f_{i j}\right]=\bar{f}_{i j}(1)-\bar{f}_{i j}(0)=\int_{0}^{1} \partial_{\alpha} \bar{f}_{i j}(\alpha) \mathrm{d} \alpha,
$$

and since $\partial_{\alpha}\left[f_{i j}\right](\alpha)=\partial_{\mathbf{r}} f_{i j}\left(\mathbf{r}-(1-\alpha), \boldsymbol{c}_{i}, \mathbf{r}+\alpha \mathbf{r}_{i j}, \boldsymbol{c}_{j}, t\right) \cdot \mathbf{r}_{i j}$ we obtain

$$
\begin{aligned}
\mathcal{R}_{3}^{\mathcal{E}}=-\sum_{i, j \in \mathfrak{S}} \frac{1}{4} \int & \left(\boldsymbol{c}_{i}-\boldsymbol{v}+\boldsymbol{c}_{j}-\boldsymbol{v}\right) \cdot \mathbf{r}_{i j} \frac{\varphi_{i j}^{\prime}\left(r_{i j}\right)}{r_{i j}} \\
& \times \partial_{\mathbf{r}} f_{i j}\left(\mathbf{r}-(1-\alpha), \boldsymbol{c}_{i}, \mathbf{r}+\alpha \mathbf{r}_{i j}, \boldsymbol{c}_{j}, t\right) \cdot \mathbf{r}_{i j} \mathrm{~d} \alpha \mathrm{d} \boldsymbol{c}_{i} \mathrm{~d} \mathbf{r}_{i j} \mathrm{~d} \boldsymbol{c}_{j},
\end{aligned}
$$


and the scalar $\alpha$ must be integrated over $(0,1)$. Finally, letting $\partial_{\mathbf{r}}$ in front of the integrals and using $\partial_{\mathbf{r}}\left(\boldsymbol{c}_{i}-\boldsymbol{v}+\boldsymbol{c}_{j}-\boldsymbol{v}\right)=-2 \boldsymbol{\nabla} \boldsymbol{v}$ we obtain that $\mathcal{R}_{3}^{\mathcal{E}}=\mathcal{P}^{\mathrm{P}}: \boldsymbol{\nabla} \boldsymbol{v}+\boldsymbol{\nabla} \cdot \mathcal{Q}_{2}^{\mathrm{P}}$ where

$$
\begin{aligned}
\mathcal{Q}_{2}^{\mathrm{P}}(\mathbf{r}, t)=- & \sum_{i, j \in \mathfrak{S}} \frac{1}{4} \int \frac{\varphi_{i j}^{\prime}\left(r_{i j}\right)}{r_{i j}} \mathbf{r}_{i j} \mathbf{r}_{i j} \cdot\left(\boldsymbol{c}_{i}-\boldsymbol{v}+\boldsymbol{c}_{j}-\boldsymbol{v}\right) \\
& \times f_{i j}\left(\mathbf{r}-(1-\alpha) \mathbf{r}_{i j}, \boldsymbol{c}_{i}, \mathbf{r}+\alpha \mathbf{r}_{i j}, \boldsymbol{c}_{j}, t\right) \mathrm{d} \alpha \mathrm{d} \boldsymbol{c}_{i} \mathrm{~d} \mathbf{r}_{i j} \mathrm{~d} \boldsymbol{c}_{j},
\end{aligned}
$$

and the energy equation is established.

\section{C.2 Derivation from generalized Boltzmann equations}

The above derivation of the macroscopic equations has used the two first equations of the hierarchy. An equivalent derivation may be obtained by directly using the generalized Boltzmann equations (3.24). We denote by $\widehat{\psi}^{l}, 1 \leq l \leq \mathrm{n}_{\mathrm{s}}+3$ the $\mathrm{n}_{\mathrm{s}}+3$ first collision invariants given by $\widehat{\psi}^{l}=\left(\delta_{i l}\right)_{i \in \mathfrak{S}}, \widehat{\psi}^{\mathrm{n}_{\mathrm{s}}+\nu}=\left(m_{i}\left(c_{i \nu}-v_{\nu}\right)\right)_{i \in \mathfrak{S}}$ for $1 \leq \nu \leq 3$, and by $\mathcal{E}^{\prime}$ the differential of the energy $\mathcal{E}$ with respect to $f=\left(f_{i}\right)_{i \in \mathfrak{S}}$, that is, $\mathcal{E}^{\prime}$ is such that for any increment distribution $\delta f=\left(\delta f_{i}\right)_{i \in \mathfrak{S}}$ we have

$$
\delta \mathcal{E}=\left\langle\left\langle\mathcal{E}^{\prime}, \delta f\right\rangle\right\rangle=\sum_{i \in \mathfrak{S}} \int \frac{1}{2} m_{i}\left|\boldsymbol{c}_{i}-\boldsymbol{v}\right|^{2} \delta f_{i} \mathrm{~d} \boldsymbol{c}_{i}+\sum_{i, j \in \mathfrak{S}} \frac{1}{2} \int \varphi_{i j} \tau_{i j}\left(f_{i} \delta f_{j}+f_{j} \delta f_{i}\right) \mathrm{d} \boldsymbol{c}_{i} \mathrm{~d} \boldsymbol{r}_{i j} \mathrm{~d} \boldsymbol{c}_{j},
$$

where $\langle\langle\rangle$ is the scalar product (4.41). The governing equations are then obtained by taking the scalar product of the generalized equations $\left(\partial_{t} f_{i}+\boldsymbol{c}_{i} \cdot \partial_{\mathbf{r}_{i}}-\mathcal{J}_{i}^{(1)}\right)_{i \in \mathfrak{S}}$ by the invariants $\widehat{\psi}^{l}, 1 \leq l \leq \mathrm{n}_{\mathrm{s}}+3$ and $\mathcal{E}^{\prime}$. The derivation of the mass and momentum conservation from (3.24) is then unchanged but the derivation of the energy conservation equation is slightly modified.

The energy conservation equation first reads

$$
\begin{aligned}
& \sum_{i \in \mathfrak{S}} \int \frac{1}{2} m_{i}\left|\boldsymbol{c}_{i}-\boldsymbol{v}\right|^{2}\left(\partial_{t} f_{i}+\boldsymbol{c}_{i} \cdot \partial_{\mathbf{r}_{i}} f_{i}-\mathcal{J}_{i}^{(1)}\right) \mathrm{d} \boldsymbol{c}_{i} \\
& +\sum_{i, j \in \mathfrak{S}} \frac{1}{2} \int \varphi_{i j} \tau_{i j}\left(f_{i}\left(\partial_{t} f_{j}+\boldsymbol{c}_{j} \cdot \partial_{\mathbf{r}_{j}} f_{j}-\mathcal{J}_{j}^{(1)}\right)\right. \\
& \left.\quad+f_{j}\left(\partial_{t} f_{i}+\boldsymbol{c}_{i} \cdot \partial_{\mathbf{r}_{i}} f_{i}-\mathcal{J}_{i}^{(1)}\right)\right) \mathrm{d} \boldsymbol{c}_{i} \mathrm{~d} \mathbf{r}_{i j} \mathrm{~d} \boldsymbol{c}_{j}=0 .
\end{aligned}
$$

The kinetic terms in this relation are easily transformed into

$$
\partial \mathcal{E}^{\mathrm{K}}+\boldsymbol{\nabla} \cdot\left(\boldsymbol{v} \mathcal{E}^{\mathrm{K}}+\mathcal{Q}^{\mathrm{K}}\right)+\mathcal{P}^{\mathrm{K}}: \boldsymbol{\nabla} \boldsymbol{v}+\boldsymbol{\mathcal { R }}_{1}^{\mathcal{E}},
$$

using standard kinetic theory manipulations. Considering next the potential part of the relation (C.8), we first note that

$$
\left.\Xi_{i j}=\int \varphi_{i j} \tau_{i j}\left(f_{i} \mathcal{J}_{j}^{(1)}+f_{j} \mathcal{J}_{i}^{(1)}\right)\right) \mathrm{d} \boldsymbol{c}_{i} \mathrm{~d} \mathbf{r}_{i j} \mathrm{~d} \boldsymbol{c}_{j}=0
$$

Indeed, we may write

$$
\begin{aligned}
\Xi_{i j} & =-\int \varphi_{i j} \tau_{i j}\left(f_{i}\left(\partial_{t} f_{j}+\boldsymbol{c}_{j} \cdot \partial_{\mathbf{r}_{j}} f_{j}\right)+f_{j}\left(\partial_{t} f_{i}+\boldsymbol{c}_{i} \cdot \partial_{\mathbf{r}_{j}} f_{i}\right)\right) \mathrm{d} \boldsymbol{c}_{i} \mathrm{~d} \mathbf{r}_{i j} \mathrm{~d} \boldsymbol{c}_{j} \\
& =-\int \varphi_{i j} \tau_{i j}\left(\partial_{t}\left(f_{i} f_{j}\right)+\boldsymbol{c}_{i} \cdot \partial_{\mathbf{r}_{i}}\left(f_{i} f_{j}\right)+\boldsymbol{c}_{j} \cdot \partial_{\mathbf{r}_{j}}\left(f_{i} f_{j}\right)\right) \mathrm{d} \boldsymbol{c}_{i} \mathrm{~d} \mathbf{r}_{i j} \mathrm{~d} \boldsymbol{c}_{j} .
\end{aligned}
$$


We may then use the identity

$$
\tau_{i j} \partial_{t}=\partial_{t} \tau_{i j}
$$

since $\tau_{i j}$ is independent of time from (3.22) as well as the commutation relation

$$
\tau_{i j}\left(\boldsymbol{c}_{i} \cdot \partial_{\mathbf{r}_{i}}+\boldsymbol{c}_{j} \cdot \partial_{\mathbf{r}_{j}}\right)=\left(\boldsymbol{c}_{i} \cdot \partial_{\mathbf{r}_{i}}+\boldsymbol{c}_{j} \cdot \partial_{\mathbf{r}_{j}}-\theta_{i j}\right) \tau_{i j},
$$

established by Green [102] using the definition (3.22). These relations now yields

$$
\Xi_{i j}=-\int \varphi_{i j}\left(\partial_{t}+\boldsymbol{c}_{i} \cdot \partial_{\mathbf{r}_{i}}+\boldsymbol{c}_{j} \cdot \partial_{\mathbf{r}_{j}}-\theta_{i j}\right) \tau_{i j} f_{i} f_{j} \mathrm{~d} \boldsymbol{c}_{i} \mathrm{~d} \mathbf{r}_{i j} \mathrm{~d} \boldsymbol{c}_{j}=0
$$

since by definition of $\tau_{i j} f_{i} f_{j}=\exp \left(-t \mathfrak{H}_{i j}\right) f_{i}(0) f_{j}(0)$ we have $\left(\partial_{t}+\boldsymbol{c}_{i} \cdot \partial_{\mathbf{r}_{i}}+\boldsymbol{c}_{j} \cdot \partial_{\mathbf{r}_{j}}-\theta_{i j}\right) f_{i j}=$ 0 . The potential remainder of the energy equation (C.8) then reads after some algebra

$$
\begin{aligned}
\sum_{i, j \in \mathfrak{S}} & \frac{1}{2} \int \varphi_{i j} \tau_{i j}\left(f_{i}\left(\partial_{t} f_{j}+\boldsymbol{c}_{j} \cdot \partial_{\mathbf{r}_{j}} f_{j}\right)+f_{j}\left(\partial_{t} f_{i}+\boldsymbol{c}_{i} \cdot \partial_{\mathbf{r}_{i}} f_{i}\right)\right) \mathrm{d} \boldsymbol{c}_{i} \mathrm{~d} \mathbf{r}_{i j} \mathrm{~d} \boldsymbol{c}_{j} \\
= & \sum_{i, j \in \mathfrak{S}} \frac{1}{2} \int \varphi_{i j}\left(\partial_{t}+\boldsymbol{c}_{i} \cdot \partial_{\mathbf{r}_{i}}+\boldsymbol{c}_{j} \cdot \partial_{\mathbf{r}_{j}}-\theta_{i j}\right) \tau_{i j} f_{i} f_{j} \mathrm{~d} \boldsymbol{c}_{i} \mathrm{~d} \mathbf{r}_{i j} \mathrm{~d} \boldsymbol{c}_{j} \\
& =\partial_{t} \mathcal{E}^{\mathrm{P}}+\boldsymbol{\nabla} \cdot\left(\boldsymbol{v} \mathcal{E}^{\mathrm{P}}+\mathcal{Q}_{2}^{\mathrm{P}}\right)+\mathcal{P}^{\mathrm{P}}: \boldsymbol{\nabla} \boldsymbol{v}-\boldsymbol{\mathcal { R }}_{1}^{\mathcal{E}},
\end{aligned}
$$

and the equation for the conservation of energy is then a consequence of (C.8) with (C.9) and (C.12).

\section{Normal linearized kinetic equations}

We evaluate in this section the linearized equations for nonideal fluid with normal diffusive processes, that is, we evaluate the right hand sides by solely using first order Taylor expansions of pair distribution functions. The thermodynamic properties are those from Section 4 without diffuse interface terms and are denoted with the superscript ${ }^{\mathrm{u}}$.

The right hand sides (4.20) are in the form

$$
\psi_{i}=-\left(\partial_{t} \log f_{i}^{(0)}+\boldsymbol{c}_{i} \cdot \nabla_{\mathbf{r}_{i}} \log f_{i}^{(0)}\right)+\frac{1}{f_{i}^{(0)}} \mathcal{J}_{i}^{(1)}\left(f^{(0)}\right), \quad i \in \mathfrak{S},
$$

and the differential expression $\partial_{t} \log f_{i}^{(0)}+\boldsymbol{c}_{i} \cdot \nabla_{\mathbf{r}_{i}} \log f_{i}^{(0)}$ may be split as $\partial_{t} \log f_{i}^{(0)}+$ $\boldsymbol{v} \cdot \nabla_{\mathbf{r}_{i}} \log f_{i}^{(0)}+\left(\boldsymbol{c}_{i}-\boldsymbol{v}\right) \cdot \nabla_{\mathbf{r}_{i}} \log f_{i}^{(0)}$. The material derivatives $\partial_{t} \log f_{i}^{(0)}+\boldsymbol{v} \cdot \nabla_{\mathbf{r}_{i}} \log f_{i}^{(0)}$ are evaluated by using the expression of Maxwellian distributions and the multicomponent Euler equations that are obtained in the form

$$
\begin{aligned}
& \partial_{t} n_{i}+\boldsymbol{v} \cdot \boldsymbol{\nabla} n_{i}=-n_{i} \boldsymbol{\nabla} \cdot \boldsymbol{v}, \\
& \partial_{t} \boldsymbol{v}+\boldsymbol{v} \cdot \boldsymbol{\nabla} \boldsymbol{v}=-\boldsymbol{\nabla} p^{\mathrm{u}} / \rho, \\
& \partial_{t} T+\boldsymbol{v} \cdot \boldsymbol{\nabla} T=\frac{1}{\partial_{T} \mathcal{E}^{\mathrm{u}}}\left(\sum_{l \in \mathfrak{S}} n_{l} \partial_{n_{l}} \mathcal{E}^{\mathrm{u}}-\mathcal{E}^{\mathrm{u}}-p^{\mathrm{u}}\right) \boldsymbol{\nabla} \cdot \boldsymbol{v} .
\end{aligned}
$$

The resulting differential expressions are in the form

$$
\begin{aligned}
-\left(\partial_{t} \log f_{i}^{(0)}\right. & \left.+\boldsymbol{v} \cdot \nabla_{\mathbf{r}_{i}} \log f_{i}^{(0)}\right)=\boldsymbol{\nabla} \cdot \boldsymbol{v}+\frac{m_{i}}{\rho k_{\mathrm{B}} T}\left(\boldsymbol{c}_{i}-\boldsymbol{v}\right) \cdot \boldsymbol{\nabla} p^{\mathrm{u}} \\
& +\left(\frac{3}{2}-\frac{m_{i}\left|\boldsymbol{c}_{i}-\boldsymbol{v}\right|^{2}}{2 k_{\mathrm{B}} T}\right) \frac{1}{T \partial_{T} \mathcal{E}^{\mathrm{u}}}\left(\sum_{l \in \mathfrak{S}} n_{l} \partial_{n_{l}} \mathcal{E}^{\mathrm{u}}-\mathcal{E}^{\mathrm{u}}-p^{\mathrm{u}}\right) \boldsymbol{\nabla} \cdot \boldsymbol{v}
\end{aligned}
$$


and

$$
\begin{aligned}
-\left(\boldsymbol{c}_{i}-\boldsymbol{v}\right) \cdot \nabla_{\mathbf{r}_{i}} \log f_{i}^{(0)}= & -\left(\boldsymbol{c}_{i}-\boldsymbol{v}\right) \cdot \frac{\boldsymbol{\nabla} n_{i}}{n_{i}}-\frac{m_{i}}{k_{\mathrm{B}} T}\left(\boldsymbol{c}_{i}-\boldsymbol{v}\right) \otimes\left(\boldsymbol{c}_{i}-\boldsymbol{v}\right): \boldsymbol{\nabla} \boldsymbol{v} \\
& +\left(\frac{3}{2}-\frac{m_{i}\left|\boldsymbol{c}_{i}-\boldsymbol{v}\right|^{2}}{2 k_{\mathrm{B}} T}\right)\left(\boldsymbol{c}_{i}-\boldsymbol{v}\right) \cdot \frac{\boldsymbol{\nabla} T}{T} .
\end{aligned}
$$

The terms arising from the collision operators $\mathcal{J}_{i}^{(1)}\left(f^{(0)}\right)$ are next evaluated from the differential expressions (3.45) and (3.46) of Section 3.4. Indeed, we deduce from Section 3.4 that at first order in the gradients, we have

$$
\boldsymbol{\nabla} f_{i j}^{(0), \mathrm{cl}}\left(\alpha \mathbf{r}_{i}+(1-\alpha) \mathbf{r}_{i j}^{\mathrm{m}}, \boldsymbol{c}_{i}, \boldsymbol{c}_{j}, t\right)=\nabla f_{i j}^{(0), \mathrm{cl}}\left(\mathbf{r}_{i}, \boldsymbol{c}_{i}, \boldsymbol{c}_{j}, t\right) .
$$

Therefore $\mathcal{J}_{i}^{(1), s}\left(f^{(0)}\right)$ reads

$$
\mathcal{J}_{i}^{(1), s}\left(f^{(0)}\right)=\sum_{j \in \mathfrak{S}} \boldsymbol{\nabla} \cdot \int \mu_{j} \theta_{i j} f_{i}^{(0)}\left(\mathbf{r}_{i}, \boldsymbol{c}_{i}, t\right) f_{j}^{(0)}\left(\mathbf{r}_{i}, \boldsymbol{c}_{j}, t\right) g_{i j}\left(\mathbf{r}_{i}, r_{i}\right) \mathbf{r}_{i j} \mathrm{~d} \boldsymbol{x}_{j},
$$

and after some calculus

$$
\mathcal{J}_{i}^{(1), s}\left(f^{(0)}\right)=\nabla \cdot\left(\sum_{j \in \mathfrak{S}} \frac{m_{j} n_{j}}{m_{i}+m_{j}} \beta_{i j} f_{i}^{(0)}\left(\boldsymbol{c}_{i}-\boldsymbol{v}\right)\right) .
$$

Similarly, we have from Section 3.4 that at first order in the gradients, we have

$$
\begin{array}{r}
\mathcal{J}_{i}^{(1), a}\left(f^{(0)}\right)=-\sum_{j \in \mathfrak{S}} \mu_{i j} \int \theta_{i j}\left(\frac{1}{m_{i}} \partial_{\mathbf{r}} f_{i}^{(0)}\left(\mathbf{r}_{i}, \boldsymbol{c}_{i}^{\prime}, t\right) f_{j}^{(0)}\left(\mathbf{r}_{i}, \boldsymbol{c}_{j}^{\prime}, t\right)\right. \\
\left.-f_{i}^{(0)}\left(\mathbf{r}_{i}, \boldsymbol{c}_{i}^{\prime}, t\right) \frac{1}{m_{j}} \partial_{\mathbf{r}} f_{j}^{(0)}\left(\mathbf{r}_{i}, \boldsymbol{c}_{j}^{\prime}, t\right) .\right) \cdot \mathbf{r}_{i j}^{\prime} \mathrm{d} \boldsymbol{x}_{j} .
\end{array}
$$

After some calculus we may write

$$
\begin{gathered}
\frac{1}{f_{i}^{(0)}} \mathcal{J}_{i}^{(1), a}\left(f^{(0)}\right)=-\sum_{j \in \mathfrak{S}} \mu_{i j} n_{j}\left(\overline{\mathfrak{a}}_{i j}^{\eta}: \nabla \boldsymbol{v}+\overline{\mathfrak{a}}_{i j}^{\kappa} \nabla \cdot \boldsymbol{v}+\overline{\mathfrak{a}}_{i j}^{\widehat{\lambda}} \cdot \nabla\left(\frac{-1}{k_{\mathrm{B}} T}\right)\right. \\
\left.+\overline{\mathfrak{a}}_{i j}^{D} \cdot\left(\frac{\boldsymbol{\nabla} n_{i}}{m_{i} n_{i}}-\frac{\boldsymbol{\nabla} n_{j}}{m_{j} n_{j}}\right)\right)
\end{gathered}
$$

where the collision integrals for dense gases $\overline{\mathfrak{a}}_{i j}^{\eta}, \overline{\mathfrak{a}}_{i j}^{\kappa}, \overline{\mathfrak{a}}_{i j}^{\widehat{\lambda}}$, and $\overline{\mathfrak{a}}_{i j}^{D}$ are defined by

$$
\begin{aligned}
\widetilde{f}_{i}^{(0)} \overline{\mathfrak{a}}_{i j}^{\eta} & =\frac{1}{2 k_{\mathrm{B}} T} \int \theta_{i j} \tau_{i j} \widetilde{f}_{i}^{(0)} \widetilde{f}_{j}^{(0)}\left(\mathbf{r}_{i j} \otimes\left(\boldsymbol{c}_{i}-\boldsymbol{c}_{j}\right)+\left(\boldsymbol{c}_{i}-\boldsymbol{c}_{j}\right) \otimes \mathbf{r}_{i j}-\frac{2}{3}\left(\boldsymbol{c}_{i}-\boldsymbol{c}_{j}\right) \cdot \mathbf{r}_{i j} \boldsymbol{I}\right) \mathrm{d} \boldsymbol{x}_{j}, \\
\widetilde{f}_{i}^{(0)} \overline{\mathfrak{a}}_{i j}^{\kappa} & =\frac{1}{3 k_{\mathrm{B}} T} \int \theta_{i j} \tau_{i j} \widetilde{f}_{i}^{(0)} \widetilde{f}_{j}^{(0)} \mathbf{r}_{i j} \cdot\left(\boldsymbol{c}_{i}-\boldsymbol{c}_{j}\right) \mathrm{d} \boldsymbol{x}_{j}, \\
\widetilde{f}_{i}^{(0)} \overline{\mathfrak{a}}_{i j}^{D} & =\int \theta_{i j} \tau_{i j} \widetilde{f}_{i}^{(0)} \widetilde{f}_{j}^{(0)} \mathbf{r}_{i j} \mathrm{~d} \boldsymbol{x}_{j}, \\
\widetilde{f}_{i}^{(0)} \overline{\mathfrak{a}}_{i j}^{\widehat{\lambda}} & =\int \theta_{i j} \tau_{i j} \widetilde{f}_{i}^{(0)} \widetilde{f}_{j}^{(0)} \mathbf{r}_{i j}\left(\frac{3}{2} \frac{k_{\mathrm{B}} T}{m_{j}}-\frac{1}{2}\left|\boldsymbol{c}_{j}-\boldsymbol{v}\right|^{2}-\frac{3}{2} \frac{k_{\mathrm{B}} T}{m_{i}}+\frac{1}{2}\left|\boldsymbol{c}_{i}-\boldsymbol{v}\right|^{2}\right) \mathrm{d} \boldsymbol{x}_{j} .
\end{aligned}
$$

The expressions (D.5) and (D.6) as well as (D.9) are essentially similar to those presented by Cohen et al. [50]. 
We now further assume that the repulsive potentials between particles are hard and use the corresponding value for the integrals $\overline{\mathfrak{a}}_{i j}^{D}$. The integrals $\overline{\mathfrak{a}}_{i j}^{D}$ may indeed be written

$$
\overline{\mathfrak{a}}_{i j}^{D}=\frac{1}{\widetilde{f}_{i}^{(0)}} \int \theta_{i j} g_{i j} \widetilde{f}_{i}^{(0)} \widetilde{f}_{j}^{(0)} \mathbf{r}_{i j}^{\prime} \mathrm{d} \boldsymbol{x}_{j}
$$

and assuming that the interaction potentials $\varphi_{i j}$ are hard, we may use the rigid sphere relation $\mathbf{r}_{i j}^{\prime}=\mathbf{r}_{i j}$ in (D.10), discussed in particular by Hoffman and Curtiss [72]. A straightforward calculation then yields

$$
\overline{\mathfrak{a}}_{i j}^{D}=\beta_{i j}\left(\boldsymbol{c}_{i}-\boldsymbol{v}\right) .
$$

More generally, for functions $A\left(\mathbf{r}_{i j}, \mathbf{r}_{i j}^{\prime}\right)$, depending on $\mathbf{r}_{i j}$ and $\mathbf{r}_{i j}^{\prime}$, we may occasionally use the hard potential relation

$$
\int \theta_{i j} g_{i j} \widetilde{f}_{i}^{(0)} \widetilde{f}_{j}^{(0)} A\left(\mathbf{r}_{i j}, \mathbf{r}_{i j}^{\prime}\right) \mathrm{d} \mathbf{r}_{i j}=\int \theta_{i j} g_{i j} \widetilde{f}_{i}^{(0)} \widetilde{f}_{j}^{(0)} A\left(\mathbf{r}_{i j}, \mathbf{r}_{i j}\right) \mathrm{d} \mathbf{r}_{i j} .
$$

With the hard potential relation (D.11), there is a simplification for density gradient and pressure terms in $\psi_{i}^{(1)}$ that are obtained in the form

$$
-\left(\boldsymbol{c}_{i}-\boldsymbol{v}\right) \cdot\left(\frac{\nabla n_{i}}{n_{i}}-\sum_{j \in \mathfrak{S}} \beta_{i j} \nabla n_{j}-\frac{m_{i}}{\rho k_{\mathrm{B}} T} \nabla p\right) .
$$

We then use that

$$
\nabla\left(\frac{g_{i}^{\mathrm{u}}}{k_{\mathrm{B}} T}\right)=\frac{\nabla n_{i}}{n_{i}}-\sum_{j \in \mathfrak{S}} \beta_{i j} \nabla n_{j}-\left(\frac{3}{2}+\sum_{j \in \mathfrak{S}} n_{j} \beta_{i j}^{\prime} k_{\mathrm{B}} T\right) \nabla T,
$$

and

$$
\sum_{k \in \mathfrak{S}} n_{k} k_{\mathrm{B}} T \nabla\left(\frac{g_{k}^{\mathrm{u}}}{k_{\mathrm{B}} T}\right)=\nabla p-\left(\mathcal{E}^{\mathrm{u}}+p^{\mathrm{u}}\right) \frac{\nabla T}{T},
$$

in order to rewrite $\psi_{i}^{(1)}$ as well as the natural thermodynamic force terms for dissipative fluids without capillary effects

$$
\boldsymbol{X}_{i}^{\mathrm{th}}=n_{i} k_{\mathrm{B}} T \nabla\left(\frac{g_{i}^{\mathrm{u}}}{k_{\mathrm{B}} T}\right)=\rho_{i} T \nabla\left(\frac{\bar{g}_{i}^{\mathrm{u}}}{T}\right) .
$$

By combining the relations (D.5), (D.6), (D.9), (D.11), (D.13) and (D.14), it is obtained after lengthy algebra that

$$
\psi_{i}^{(1)}=-\psi_{i}^{\eta}: \nabla \boldsymbol{v}-\psi_{i}^{\kappa} \nabla \cdot \boldsymbol{v}-\sum_{j \in \mathfrak{S}} \psi_{i}^{D_{j}} \cdot \boldsymbol{X}_{j}^{\mathrm{th}}-\psi_{i}^{\widehat{\lambda}} \cdot \nabla\left(\frac{-1}{k_{\mathrm{B}} T}\right),
$$

which is relation (4.21), together with the expressions of $\psi_{i}^{\eta}, \psi_{i}^{\kappa}, \psi^{D_{j}}, j \in \mathfrak{S}$, and $\psi_{i}^{\widehat{\lambda}}$, given in Section 4.4.

We denote by $\widehat{\psi}^{l}, 1 \leq l \leq \mathrm{n}_{\mathrm{s}}+4$ the $\mathrm{n}_{\mathrm{s}}+4$ collisional invariants given by $\widehat{\psi}^{l}=\left(\delta_{i l}\right)_{i \in \mathfrak{S}}$, $\widehat{\psi}^{\mathrm{n}_{\mathrm{s}}+\nu}=\left(m_{i}\left(c_{i \nu}-v_{\nu}\right)\right)_{i \in \mathfrak{S}}$ for $1 \leq \nu \leq 3$, and $\widehat{\psi}^{\mathrm{n}_{\mathrm{s}}+4}=\left(\frac{1}{2} m_{i}\left|\boldsymbol{c}_{i}-\boldsymbol{v}\right|^{2}\right)_{i \in \mathfrak{S}}$, and by $\langle\langle\rangle\rangle$ the scalar product (4.41). The tensorial right hand sides $\psi_{i}^{\eta}, \psi_{i}^{\kappa}, \psi^{D_{j}}, j \in \mathfrak{S}$, and $\psi_{i}^{\widehat{\lambda}}$, given in Section 4.4, then satisfy the orthogonality relations

$$
\left\langle\left\langle\psi^{\mu}, \widehat{\psi}^{l}\right\rangle\right\rangle=0
$$


for $\mu \in \eta, \kappa, D_{j}, j \in \mathfrak{S}, \hat{\lambda}$ and $1 \leq l \leq \mathrm{n}_{\mathrm{s}}+4$. These relations are easily established excepted for the energy for which it is necessary to use the approximation [50]

$$
n k_{\mathrm{B}} T+\frac{\frac{3}{2} n k_{\mathrm{B}}}{\partial_{T} \mathcal{E}^{\mathrm{u}}}\left(\sum_{l \in \mathfrak{S}} n_{l} \partial_{n_{l}} \mathcal{E}^{\mathrm{u}}-\mathcal{E}^{\mathrm{u}}-p^{\mathrm{u}}\right)=\sum_{i, j \in \mathfrak{S}} n_{i} n_{j}\left(\frac{1}{2} \beta_{i j} k_{\mathrm{B}} T+\frac{7}{6} \beta_{i j}^{\prime}\left(k_{\mathrm{B}} T\right)^{2}+\frac{1}{3} \beta_{i j}^{\prime \prime}\left(k_{\mathrm{B}} T\right)^{3}\right),
$$

as well as the identity [50]

$$
\sum_{i, j \in \mathfrak{S}} \mu_{i j} n_{i} n_{j} \int \frac{1}{2} m_{i}\left|\boldsymbol{c}_{i}-\boldsymbol{v}\right|^{2} \widetilde{f}_{i}^{(0)} \overline{\mathfrak{a}}_{i j}^{\kappa} \mathrm{d} \boldsymbol{c}_{i}=-\sum_{i, j \in \mathfrak{S}} n_{i} n_{j}\left(\frac{7}{6} \beta_{i j}^{\prime}\left(k_{\mathrm{B}} T\right)^{2}+\frac{1}{3} \beta_{i j}^{\prime \prime}\left(k_{\mathrm{B}} T\right)^{3}\right),
$$

established notably in Chaos and Colin [103].

\section{E Constraints for perturbed distribution functions}

The perturbed distribution functions $\phi_{i}^{(1)}, i \in \mathfrak{S}$, are uniquely determined by imposing Enskog type constraints. These constraints insure that the true value of the local macroscopic variables $n_{i}, \boldsymbol{v}$ and $T$, or equivalently $\mathcal{E}$, are determined by $f_{i}^{(0)}$ alone $[57,54,50]$.

The constraints associated with mass and momentum are identical to that for dilute gases $\int f_{i}^{(0)} \phi_{i}^{(1)} \mathrm{d} \boldsymbol{c}_{i}=0$ for $i \in \mathfrak{S}$, and $\sum_{i \in \mathfrak{S}} \int m_{i}\left(\boldsymbol{c}_{i}-\boldsymbol{v}\right) f_{i}^{(0)} \phi_{i}^{(1)} \mathrm{d} \boldsymbol{c}_{i}=0$ and corresponds to (4.29) and (4.30).

The constraint associated with internal energy is more complex since it involves pair distribution functions. The Enskog contraint for energy is generally in the form

$$
\sum_{i \in \mathfrak{S}} \int \frac{1}{2} m_{i}\left|\boldsymbol{c}_{i}-\boldsymbol{v}\right|^{2} f_{i}^{(0)} \phi_{i}^{(1)} \mathrm{d} \boldsymbol{c}_{i}+\sum_{i, j \in \mathfrak{S}} \int \frac{1}{2} \varphi_{i j}\left(f_{i j}-f_{i j}^{(0)}\right) \mathrm{d} \mathbf{r}_{i j} \mathrm{~d} \boldsymbol{c}_{i} \mathrm{~d} \boldsymbol{c}_{j}=0,
$$

where the $f_{i j}$ denote the nonequilibrium pair distribution functions, $f_{i j}^{(0)}$ the zeroth order pair distribution functions, and where the $f_{i j}$ are evaluated with $f_{i}=f_{i}^{(0)}+f_{i}^{(0)} \phi_{i}^{(1)}$. The kinetic part of the constraint (E.1) is thus traditional whereas the potential part depends on the nonequilibrium pair distribution functions $f_{i j}$, the zeroth order pair distribution functions $f_{i j}^{(0)}$, as well as on the order of Taylor expansions.

Considering first the kinetic theory of nonideal fluids with normal diffusive processes presented in Section 4, the potential part of the constraint is in the form

$$
\sum_{i, j \in \mathfrak{S}} \int \frac{1}{2} \varphi_{i j}\left(f_{i j}^{\mathrm{Bo}}-f_{i j}^{(0), \mathrm{cl}}\right) \mathrm{d} \mathbf{r}_{i j} \mathrm{~d} \boldsymbol{c}_{i} \mathrm{~d} \boldsymbol{c}_{j}
$$

with $f_{i j}=f_{i j}^{\mathrm{Bo}}=\tau_{i j} f_{i}\left(\mathbf{r}_{i}\right) f_{j}\left(\mathbf{r}_{j}\right), f_{i j}^{(0)}=f_{i j}^{(0), \mathrm{cl}}=g_{i j} f_{i}^{(0)}\left(\mathbf{r}_{i}\right) f_{j}^{(0)}\left(\mathbf{r}_{i}\right)$ and $f_{i}=f_{i}^{(0)}+f_{i}^{(0)} \phi_{i}^{(1)}$, $i, j \in \mathfrak{S}$. For such a kinetic theory of nonideal fluids, we may use first order Taylor expansions and neglect any gradient of first order terms $[57,54,50]$. This yields

$$
f_{i j}^{\mathrm{Bo}}-f_{i j}^{(0), \mathrm{cl}}=g_{i j} f_{i}^{(0)} f_{j}^{(0)}\left(\phi_{i}^{(1)}+\phi_{j}^{(1)}\right)+g_{i j} f_{i}^{(0)} f_{j}^{(0)}\left(\boldsymbol{\nabla} \log f_{i}^{(0)} \cdot \delta \mathbf{r}_{i}+\boldsymbol{\nabla} \log f_{j}^{(0)} \cdot \delta \mathbf{r}_{j}\right),
$$

where $\delta \mathbf{r}_{i}=\mathbf{r}_{i}^{\prime}-\mathbf{r}_{i}$ and $\delta \mathbf{r}_{j}=\mathbf{r}_{j}^{\prime}-\mathbf{r}_{i}$. The Maxwellian gradient contributions then vanish in (E.1) since they are odd with respect to $\mathbf{r}_{i j}$ or $\mathbf{r}_{i j}^{\prime}$ and the remaining terms are localized at $\mathbf{r}_{i}$ so that the constraint reads

$$
\sum_{i \in \mathfrak{S}} \int \frac{1}{2} m_{i}\left|\boldsymbol{c}_{i}-\boldsymbol{v}\right|^{2} f_{i}^{(0)} \phi_{i}^{(1)} \mathrm{d} \boldsymbol{c}_{i}+\sum_{i, j \in \mathfrak{S}} \int \frac{1}{2} \varphi_{i j} \tau_{i j} f_{i}^{(0)} f_{j}^{(0)}\left(\phi_{i}^{(1)}+\phi_{j}^{(1)}\right) \mathrm{d} \mathbf{r}_{i j} \mathrm{~d} \boldsymbol{c}_{i} \mathrm{~d} \boldsymbol{c}_{j}=0 .
$$


This constraint is further simplified by using the structure of the perturbed distribution functions for fluid mixtures $[50,51]$ as for single species fluids $[54,57]$. The only difficult terms are those associated with the potential part of the volume viscosity terms for which expansions with respect to density are generally used and the resulting constraint is obtained in the form (4.31) and is of the classical form [50, 51, 54, 57].

Considering next the kinetic theory using second order expansions of pair distribution functions that leads to capillary effects, the potential part of the energy constraint is in the form

$$
\sum_{i, j \in \mathfrak{S}} \int \frac{1}{2} \varphi_{i j}\left(f_{i j}^{\mathrm{Bo}}-f_{i j}^{(0), \mathrm{sy}}\right) \mathrm{d} \mathbf{r}_{i j} \mathrm{~d} \boldsymbol{c}_{i} \mathrm{~d} \boldsymbol{c}_{j},
$$

with $f_{i j}=f_{i j}^{\mathrm{Bo}}=\tau_{i j} f_{i}\left(\mathbf{r}_{i}\right) f_{j}\left(\mathbf{r}_{j}\right), f_{i j}^{(0)}=f_{i j}^{(0), \mathrm{sy}}=g_{i j} f_{i}\left(\mathbf{r}_{i}\right) f_{j}\left(\mathbf{r}_{j}\right)$ and $f_{i}=f_{i}^{(0)}+f_{i}^{(0)} \phi_{i}^{(1)}$, $i, j \in \mathfrak{S}$. For the kinetic theory of nonideal fluids including diffuse interface effects, we have to use second order Taylor expansions and write

$$
f_{i j}^{\mathrm{Bo}}-f_{i j}^{(0), \mathrm{cl}}=f_{i}^{(0)}\left(\mathbf{r}_{i}^{\prime}\right) f_{j}^{(0)}\left(\mathbf{r}_{j}^{\prime}\right)\left(1+\phi_{i}^{(1)}\left(\mathbf{r}_{i}^{\prime}\right)\right)\left(1+\phi_{j}^{(1)}\left(\mathbf{r}_{j}^{\prime}\right)\right)-g_{i j} f_{i}^{(0)}\left(\mathbf{r}_{i}\right) f_{j}^{(0)}\left(\mathbf{r}_{j}\right),
$$

where the dependence on particle velocities has been left implicit. Considering first the terms arising solely from the Maxwellian distributions $f_{i}^{(0)}\left(\mathbf{r}_{i}^{\prime}\right) f_{j}^{(0)}\left(\mathbf{r}_{j}^{\prime}\right)-g_{i j} f_{i}^{(0)}\left(\mathbf{r}_{i}\right) f_{j}^{(0)}\left(\mathbf{r}_{j}\right)$, and using a a second order Taylor expansion, the zeroth order terms are zero, the first order terms yield zero contributions to the energy constraint since they are odd with respect to $\mathbf{r}_{i j}$ or $\mathbf{r}_{i j}^{\prime}$ and we are left with the second order terms in the form

$$
\partial_{\left(\mathbf{r}_{i}, \mathbf{r}_{j}\right)}^{2} f_{i j}^{(0), \text { Bo }}:\left(\delta \mathbf{r}_{i}, \delta \mathbf{r}_{j}\right)^{\otimes 2}-\partial_{\mathbf{r}_{j}}^{2} f_{i j}^{(0), \text { sy }}: \mathbf{r}_{i j}^{\otimes 2}
$$

The leading terms that only involve density gradients then vanish using the hard potential relations. The remaining part are then negligible in the regime under consideration when $\left(\frac{\sigma^{\star}}{r^{\star}}\right)^{3}\left(\frac{\sigma^{\star}}{l_{\nabla n}^{\star}}\right)\left(\frac{\sigma^{\star}}{l_{\nabla T}^{\star}}\right) \ll 1$ and $\left(\frac{\sigma^{\star}}{r^{\star}}\right)^{3}\left(\frac{\sigma^{\star}}{l_{\nabla n}^{\star}}\right)\left(\frac{\sigma^{\star}}{l_{\nabla v}^{\star}}\right)\left(\frac{\delta v^{\star}}{\sqrt{k_{\mathrm{B}} T^{\star} / m^{\star}}}\right) \ll 1$, using notably the decompositions (6.21) as well as the approximate collision integrals for dense gases (D.11).

Considering then the terms involving the first order perturbations $\phi_{i}^{(1)}, i \in \mathfrak{S}$, using second order Taylor expansions, and discarding quadratic terms in first order perturbations. The zeroth order terms are then zero from the zero average constraints of the $\phi_{i}^{(1)}$, the first order terms yields zero contributions since they are odd with respect to $\mathbf{r}_{i j}$ or $\mathbf{r}_{i j}^{\prime}$ and we are left with second order terms in the form

$$
g_{i j} f_{i}^{(0)} f_{j}^{(0)} \partial_{\mathbf{r}_{i}} \phi_{i}^{(1)} \cdot \delta \mathbf{r}_{i} \partial_{\mathbf{r}_{j}} \log f_{j}^{(0)} \cdot \delta \mathbf{r}_{j}
$$

and

$$
g_{i j} f_{i}^{(0)} f_{j}^{(0)} \partial_{\mathbf{r}_{i}}^{2} \phi_{i}^{(1)}: \delta \mathbf{r}_{i}^{\otimes 2}
$$

and similar terms obtained by permuting $i$ and $j$. For the first type terms, we may replace $\partial_{\mathbf{r}_{i}} \log _{i}^{(0)} \cdot \delta \mathbf{r}_{j}$ by $\nabla \log n_{j}$ and use the zero average constraint of $\phi_{i}^{(1)}$ and the same zero average argument also applies for the second type terms so that finally the energy constraint is again reduced to the classical constraint (4.31) as for fluid with standard diffusive processes.

\section{F Tensorial integral and differential identities}

We summarize in this Appendix various tensorial integral relations used in the derivation of the capillary fluid equations. We denote by $\boldsymbol{z} \in \mathbb{R}^{3}$ an integration variable that may 
either represents the rescaled particle velocity $\left(\boldsymbol{c}_{i}-\boldsymbol{v}\right)\left(m_{i} / 2 k_{\mathrm{B}} T\right)^{1 / 2}$ of the $i$ th species or else the relative position $\mathbf{r}_{i j}=\mathbf{r}_{j}-\mathbf{r}_{i}$.

Letting $z=|\boldsymbol{z}|$ for $\boldsymbol{z} \in \mathbb{R}^{3}$ then for any isotropic function $\gamma(z)$ of $\boldsymbol{z}$, we have

$$
\int z_{i}^{2} \gamma(z) \mathrm{d} \boldsymbol{z}=\frac{1}{3} \int z^{2} \gamma(z) \mathrm{d} \boldsymbol{z}, \quad 1 \leq i \leq 3,
$$

and this implies

$$
\int \boldsymbol{z}(\boldsymbol{z} \cdot \mathfrak{a}) \gamma(z) \mathrm{d} \boldsymbol{z}=\frac{1}{3} \int z^{2} \gamma(z) \mathrm{d} \boldsymbol{z} \mathfrak{a},
$$

for any vector $\mathfrak{a}$ independent of $\boldsymbol{z}$. Similarly, we have [57]

$$
\int \boldsymbol{z} \otimes \boldsymbol{z} \gamma(z) \mathrm{d} \boldsymbol{z}=\frac{1}{3} \int z^{2} \gamma(z) \mathrm{d} \boldsymbol{z} \boldsymbol{I},
$$

where $\boldsymbol{I}$ is the identity tensor in $\mathbb{R}^{3}$ and $\boldsymbol{z} \otimes \boldsymbol{z}$ the tensor product of $\boldsymbol{z}$ with itself having components $z_{i} z_{j}$ for $1 \leq i, j \leq 3$.

In the same vein, for any isotropic function $\gamma(z)$ of $z$ we have [57]

$$
\int z_{i}^{4} \gamma(z) \mathrm{d} \boldsymbol{z}=\frac{1}{5} \int z^{4} \gamma(z) \mathrm{d} \boldsymbol{z}, \quad 1 \leq i \leq 3,
$$

as well as

$$
\int z_{i}^{2} z_{j}^{2} \gamma(z) \mathrm{d} \boldsymbol{z}=\frac{1}{15} \int z^{4} \gamma(z) \mathrm{d} \boldsymbol{z}, \quad 1 \leq i, j \leq 3, \quad i \neq j .
$$

As a consequence, for any second rank tensor $\mathbf{w}$ independent of $\boldsymbol{z}$ we have

$$
\int \gamma(z) \boldsymbol{z} \otimes \boldsymbol{z}(\boldsymbol{z} \otimes \boldsymbol{z}: \mathbf{w}) \mathrm{d} \boldsymbol{z}=\frac{1}{15} \int z^{4} \gamma(z) \mathrm{d} \boldsymbol{z}\left(\mathbf{w}+\mathbf{w}^{t}+\mathbf{w}: \boldsymbol{I} \boldsymbol{I}\right),
$$

where $\mathbf{w : v}$ is the full contraction between the two tensors $\mathbf{w}$ and $\mathbf{v}$, so that $\mathbf{w : I}$ is the trace of the tensor $\mathbf{w}$, and $\mathbf{w}^{t}$ denotes the transpose of $\mathbf{w}$. The tensor $\boldsymbol{z} \otimes \boldsymbol{z}$ is also denoted for short by $\boldsymbol{z}^{\otimes 2}$ and the tensor $\boldsymbol{z} \otimes \boldsymbol{z} \otimes \boldsymbol{z}$ by $\boldsymbol{z}^{\otimes 3}$.

More generally, for any pair of second rank tensors $\mathbf{w}$ and $\mathbf{v}$ independent of $\boldsymbol{z}$, assuming that $\mathbf{w}$ is symmetric, and for any isotropic function $\gamma(z)$ we have

$$
\int\left(\gamma(z) \mathbf{w}: \boldsymbol{z}^{\otimes 2} \mathbf{v}: \boldsymbol{z}^{\otimes 2}\right) \mathrm{d} \boldsymbol{z}=\frac{1}{15} \int z^{4} \gamma(z) \mathrm{d} \boldsymbol{z}\left(\mathbf{w}: \mathbf{v}+\mathbf{w}: \mathbf{v}^{t}+\operatorname{tr}(\mathbf{w}) \operatorname{tr}(\mathbf{v}) \boldsymbol{I}\right),
$$

where $\operatorname{tr}(\mathbf{w})$ denote the trace of a tensor $\mathbf{w}$.

Moreover, for any third rank tensor $\mathbf{A}_{i j k}$ independent of $\boldsymbol{z}$ and for any isotropic function $\gamma(z)$ we have

$$
\int\left(\gamma(z) z_{i} \mathbf{A}: \boldsymbol{z}^{\otimes 3}\right) \mathrm{d} \boldsymbol{z}=\frac{1}{15} \int z^{4} \gamma(z) \mathrm{d} \boldsymbol{z}\left(\sum_{l} \mathbf{A}_{i l l}+\sum_{l} \mathbf{A}_{l i l}+\sum_{l} \mathbf{A}_{l l i}\right),
$$

where $\mathbf{A}: \boldsymbol{z}^{\otimes 3}$ is the total contraction of $\mathbf{A}$ with the tensor product $\boldsymbol{z}^{\otimes 3}=\boldsymbol{z} \otimes \boldsymbol{z} \otimes \boldsymbol{z}$ given by $\mathbf{A}: \boldsymbol{z}^{\otimes 3}=\sum_{i j k} \mathbf{A}_{i j k} z_{i} z_{j} z_{k}$. As a typical application, for any third order gradient tensor like $\boldsymbol{\nabla}^{2} \boldsymbol{v}$ independent of $\boldsymbol{z}$ and for any isotropic function $\gamma(z)$ we have

$$
\int \gamma(z) \boldsymbol{z}\left(\boldsymbol{\nabla}^{2} \boldsymbol{v}:(\boldsymbol{z} \otimes \boldsymbol{z} \otimes \boldsymbol{z})\right) \mathrm{d} \boldsymbol{z}=\frac{1}{15} \int z^{4} \gamma(z) \mathrm{d} \boldsymbol{z}(\Delta \boldsymbol{v}+2 \boldsymbol{\nabla}(\boldsymbol{\nabla} \cdot \boldsymbol{v})),
$$

where $\nabla^{2} \boldsymbol{v}: \boldsymbol{z}^{\otimes 3}$ is the total contraction of $\boldsymbol{\nabla}^{2} \boldsymbol{v}$ with the tensor product $\boldsymbol{z}^{\otimes 3}=\boldsymbol{z} \otimes \boldsymbol{z} \otimes \boldsymbol{z}$ given by $\nabla^{2} \boldsymbol{v}: \boldsymbol{z}^{\otimes 3}=\sum_{i j k} \partial_{\mathbf{r}_{j}} \partial_{\mathbf{r}_{k}} \boldsymbol{v}_{i} z_{i} z_{j} z_{k}$. 
We now investigate various differential relations used in the derivation of the capillary fluid equations. The following relation is established for scalar functions $n_{i}(\mathbf{r})$ and $n_{j}(\mathbf{r})$, $i, j \in \mathfrak{S}$, of $\mathbf{r} \in \mathbb{R}^{3}$ after integrations by parts

$$
\boldsymbol{\nabla} \cdot\left(n_{i} \boldsymbol{\nabla}^{2} n_{j}\right)=\boldsymbol{\nabla} \cdot\left(n_{i} \Delta n_{j} \boldsymbol{I}+\boldsymbol{\nabla} n_{i} \cdot \boldsymbol{\nabla} n_{j} \boldsymbol{I}-\boldsymbol{\nabla} n_{i} \otimes \boldsymbol{\nabla} n_{j}\right),
$$

where $\boldsymbol{\nabla} n_{i}$ is the gradient of $n_{i}, \Delta n_{i}=\boldsymbol{\nabla} \cdot \boldsymbol{\nabla} n_{i}$ the Laplacian, and $\boldsymbol{\nabla}^{2} n_{i}$ the Hessian matrix with components $\partial_{\mathbf{r}_{k}} \partial_{\mathbf{r}_{l}} n$ for $1 \leq k, l \leq 3$.

In addition, for any scalar functions $n_{i}(\mathbf{r})$ and $n_{j}(\mathbf{r}), i, j \in \mathfrak{S}$, and any vector function $\boldsymbol{v}(\mathbf{r})$ of $\mathbf{r} \in \mathbb{R}^{3}$ one may establish after a few integration by parts that

$\boldsymbol{\nabla} \cdot\left(n_{i} \boldsymbol{\nabla} \cdot \boldsymbol{v} \boldsymbol{\nabla} n_{j}\right)=\boldsymbol{\nabla} \cdot\left(n_{i} \boldsymbol{\nabla} \boldsymbol{v} \cdot \boldsymbol{\nabla} n_{j}+\left(\boldsymbol{\nabla} n_{i} \cdot \boldsymbol{\nabla} n_{j} \boldsymbol{I}+n_{i} \Delta n_{j} \boldsymbol{I}-\boldsymbol{\nabla} n_{j} \otimes \boldsymbol{\nabla} n_{i}-n_{i} \boldsymbol{\nabla}^{2} n_{j}\right) \cdot \boldsymbol{v}\right)$.

where $\boldsymbol{\nabla} \boldsymbol{v}$ is the gradient matrix and $\boldsymbol{\nabla} \cdot \boldsymbol{v}$ the divergence of $\boldsymbol{v}$. Similarly, one may establish the following identity

$\boldsymbol{\nabla} \cdot\left(n_{i} n_{j} \boldsymbol{\nabla}(\boldsymbol{\nabla} \cdot \boldsymbol{v})\right)=\boldsymbol{\nabla} \cdot\left(n_{i} \boldsymbol{\nabla} \boldsymbol{v} \cdot \boldsymbol{\nabla} n_{j}+n_{j} \boldsymbol{\nabla} \boldsymbol{v} \cdot \boldsymbol{\nabla} n_{i}-n_{i} \boldsymbol{\nabla} \boldsymbol{v}^{t} \cdot \boldsymbol{\nabla} n_{j}-n_{j} \boldsymbol{\nabla} \boldsymbol{v}^{t} \cdot \boldsymbol{\nabla} n_{i}+n_{i} n_{j} \Delta \boldsymbol{v}\right)$,

where $\boldsymbol{\nabla} \boldsymbol{v}^{t}$ denotes the transpose of the gradient matrix.

Denoting then by $\mathcal{F}_{i}$ any vector function of $\mathbf{r} \in \mathbb{R}^{3}$, it is also obtained after a few integration by parts that

$$
\left.\boldsymbol{\nabla} \cdot\left(\nabla^{2} n_{j} \cdot \mathcal{F}_{i}\right)\right)=\nabla \cdot\left(\Delta n_{j} \mathcal{F}_{i}+\nabla \mathcal{F}_{i} \cdot \nabla n_{j}-\nabla n_{j} \nabla \cdot \mathcal{F}_{i}\right),
$$

and letting $n_{i}=1$ in the (F.9) we finally obtain that

$$
\boldsymbol{\nabla} \cdot\left(n_{j} \boldsymbol{\nabla}\left(\boldsymbol{\nabla} \cdot \mathcal{F}_{i}\right)\right)=\boldsymbol{\nabla} \cdot\left(\boldsymbol{\nabla} \mathcal{F}_{i} \cdot \nabla n_{j}-\nabla \mathcal{F}_{i}^{t} \cdot \nabla n_{j}+n_{j} \Delta \mathcal{F}_{i}\right)
$$

\section{G Other Bogoliubov deviation terms}

We complete in this Appendix the estimates of the deviations arising from the differences $f_{i j}^{\text {Bo }}-f_{i j}^{\text {sy }}$ by investigating the pressure tensor $\mathcal{P}$ and the heat fluxes $\mathcal{Q}_{1}^{\mathrm{P}}$ and $\mathcal{Q}_{2}^{\mathrm{P}}$.

The corrections for the pressure tensor $\delta^{\mathrm{Bo}} \mathcal{P}=\delta^{\mathrm{Bo}} \mathcal{P}^{\mathrm{P}}$ are first written in the form

$$
\delta^{\mathrm{Bo}} \mathcal{P}=-\sum_{i, j \in \mathfrak{S}} \frac{1}{2} \int \frac{\varphi_{i j}^{\prime}\left(r_{i j}\right)}{r_{i j}} \mathbf{r}_{i j} \otimes \mathbf{r}_{i j}\left(f_{i j}^{(0), \mathrm{Bo}}-f_{i j}^{(0), \mathrm{sy}}\right)\left(\mathbf{r}_{i}^{\alpha}, \mathbf{r}_{j}^{\alpha}\right) \mathrm{d} \alpha \mathrm{d} \boldsymbol{c}_{i} \mathrm{~d} \mathbf{r}_{i j} \mathrm{~d} \boldsymbol{c}_{j},
$$

where we have denoted for convenience $\mathbf{r}_{i}^{\alpha}=\mathbf{r}_{i}-(1-\alpha) \mathbf{r}_{i j}$ and $\mathbf{r}_{j}^{\alpha}=\mathbf{r}_{i}+\alpha \mathbf{r}_{i j}$ and have left implicit the dependence of $f_{i j}^{(0) \text {,Bo }}-f_{i j}^{(0), \text { sy }}$ on the velocities $\boldsymbol{c}_{i}$ and $\boldsymbol{c}_{j}$ We may thus write that

$$
\begin{aligned}
\delta^{\mathrm{Bo}} \mathcal{P}=-\sum_{i, j \in \mathfrak{S}} \int \frac{1}{4} \frac{\varphi_{i j}^{\prime}\left(r_{i j}\right)}{r_{i j}} \mathbf{r}_{i j} \otimes \mathbf{r}_{i j}\left(\partial_{\left(\mathbf{r}_{i}, \mathbf{r}_{j}\right)}^{2} f_{i j}^{(0), \mathrm{Bo}}:\left(\delta \mathbf{r}_{i}^{\alpha}, \delta \mathbf{r}_{j}^{\alpha}\right)^{\otimes 2}\right. \\
\left.-\partial_{\mathbf{r}_{i}, \mathbf{r}_{j}}^{2} f_{i j}^{(0), \mathrm{sy}}:\left(-\mathbf{r}_{i j}+\alpha \mathbf{r}_{i j}, \alpha \mathbf{r}_{i j}\right)^{\otimes 2}\right) \mathrm{d} \alpha \mathrm{d} \boldsymbol{c}_{i} \mathrm{~d} \mathbf{r}_{i j} \mathrm{~d} \boldsymbol{c}_{j},
\end{aligned}
$$

where $\delta \mathbf{r}_{i}^{\alpha}=\mathbf{r}_{i}^{\prime \alpha}-\mathbf{r}_{i}=\mathbf{r}_{i}^{\prime}-(1-\alpha) \mathbf{r}_{i j}^{\prime}-\mathbf{r}_{i}$ and $\delta \mathbf{r}_{j}^{\alpha}=\mathbf{r}_{j}^{\prime \alpha}-\mathbf{r}_{i}=\mathbf{r}_{i}^{\prime}+\alpha \mathbf{r}_{i j}^{\prime}-\mathbf{r}_{i}=$ $\mathbf{r}_{j}^{\prime}-(1-\alpha) \mathbf{r}_{i j}^{\prime}-\mathbf{r}_{i}$. Using the identity

$$
\int_{0}^{1}(a+\alpha b)(c+\alpha d) \mathrm{d} \alpha=\left(a+\frac{1}{2} b\right)\left(c+\frac{1}{2} d\right)+\frac{1}{12} b d,
$$


with $\delta \mathbf{r}_{i}^{\alpha}=\delta \mathbf{r}_{i}-\mathbf{r}_{i j}^{\prime}+\alpha \mathbf{r}_{i j}^{\prime}$ and $\delta \mathbf{r}_{j}^{\alpha}=\delta \mathbf{r}_{i}+\alpha \mathbf{r}_{i j}^{\prime}$, and integrating with respect to $\alpha$, it is obtained that

$$
\begin{aligned}
\delta^{\mathrm{Bo}} \mathcal{P}=-\sum_{i, j \in \mathfrak{S}} \int \frac{1}{4} \frac{\varphi_{i j}^{\prime}\left(r_{i j}\right)}{r_{i j}} \mathbf{r}_{i j} \otimes \mathbf{r}_{i j}\left(\partial_{\left(\mathbf{r}_{i}, \mathbf{r}_{j}\right)}^{2} f_{i j}^{(0), \mathrm{Bo}}:\left(\delta \mathbf{r}_{i}-\frac{1}{2} \mathbf{r}_{i j}^{\prime}, \delta \mathbf{r}_{i}+\frac{1}{2} \mathbf{r}_{i j}^{\prime}\right)^{\otimes 2}\right. \\
\quad-\partial_{\left(\mathbf{r}_{i}, \mathbf{r}_{j}\right)}^{2} f_{i j}^{(0), \mathrm{sy}}:\left(-\frac{1}{2} \mathbf{r}_{i j}, \frac{1}{2} \mathbf{r}_{i j}\right)^{\otimes 2} \\
\quad+\frac{1}{12} \partial_{\left(\mathbf{r}_{i}, \mathbf{r}_{j}\right)}^{2} f_{i j}^{(0), \mathrm{Bo}}:\left(\frac{1}{2} \mathbf{r}_{i j}^{\prime}, \frac{1}{2} \mathbf{r}_{i j}^{\prime}\right)^{\otimes 2} \\
\left.\quad-\frac{1}{12} \partial_{\left(\mathbf{r}_{i}, \mathbf{r}_{j}\right)}^{2} f_{i j}^{(0), \mathrm{sy}}:\left(\frac{1}{2} \mathbf{r}_{i j}, \frac{1}{2} \mathbf{r}_{i j}\right)^{\otimes 2}\right) \mathrm{d} \alpha \mathrm{d} \boldsymbol{c}_{i} \mathrm{~d} \mathbf{r}_{i j} \mathrm{~d} \boldsymbol{c}_{j}
\end{aligned}
$$

The dominant correction terms in $\delta^{\mathrm{Bo}} \mathcal{P}$ may then be split into $\delta^{\mathrm{Bo}} \mathcal{P}_{1}$ and $\delta^{\mathrm{Bo}} \mathcal{P}_{2}$ with

$$
\begin{aligned}
& \delta^{\mathrm{Bo}} \mathcal{P}_{1}=-\sum_{i, j \in \mathfrak{S}} \int \frac{1}{4} \frac{\varphi_{i j}^{\prime}\left(r_{i j}\right)}{r_{i j}} \mathbf{r}_{i j} \otimes \mathbf{r}_{i j} g_{i j} f_{i}^{(0)} f_{j}^{(0)}\left(\frac{\partial_{\mathbf{r}_{i}}^{2} n_{i}}{n_{i}}:\left(\left(\delta \mathbf{r}_{i}-\frac{1}{2} \mathbf{r}_{i j}^{\prime}\right)^{\otimes 2}-\left(-\frac{1}{2} \mathbf{r}_{i j}\right)^{\otimes 2}\right)\right. \\
& 2 \frac{\partial_{\mathbf{r}_{i}} n_{i}}{n_{i}} \cdot\left(\delta \mathbf{r}_{i}-\frac{1}{2} \mathbf{r}_{i j}^{\prime}\right) \frac{\partial_{\mathbf{r}_{j}} n_{j}}{n_{j}} \cdot\left(\delta \mathbf{r}_{i}+\frac{1}{2} \mathbf{r}_{i j}^{\prime}\right)-2 \frac{\partial_{\mathbf{r}_{i}} n_{i}}{n_{i}} \cdot\left(-\frac{1}{2} \mathbf{r}_{i j}\right) \frac{\partial_{\mathbf{r}_{j}} n_{j}}{n_{j}} \cdot\left(+\frac{1}{2} \mathbf{r}_{i j}\right) \\
& \left.+\frac{\partial_{\mathbf{r}_{j}}^{2} n_{j}}{n_{j}}:\left(\left(\delta \mathbf{r}_{i}+\frac{1}{2} \mathbf{r}_{i j}^{\prime}\right)^{\otimes 2}-\left(\frac{1}{2} \mathbf{r}_{i j}\right)^{\otimes 2}\right)\right) \mathrm{d} \boldsymbol{c}_{i} \mathrm{~d} \mathbf{r}_{i j} \mathrm{~d} \boldsymbol{c}_{j},
\end{aligned}
$$

and

$$
\begin{aligned}
& \delta^{\mathrm{Bo}} \mathcal{P}_{2}=-\sum_{i, j \in \mathfrak{S}} \int \frac{1}{48} \frac{\varphi_{i j}^{\prime}\left(r_{i j}\right)}{r_{i j}} \mathbf{r}_{i j} \otimes \mathbf{r}_{i j} g_{i j} f_{i}^{(0)} f_{j}^{(0)}\left(\frac{\partial_{\mathbf{r}_{i}}^{2} n_{i}}{n_{i}}:\left(\left(\frac{1}{2} \mathbf{r}_{i j}^{\prime}\right)^{\otimes 2}-\left(\frac{1}{2} \mathbf{r}_{i j}\right)^{\otimes 2}\right)\right. \\
& 2 \frac{\partial_{\mathbf{r}_{i}} n_{i}}{n_{i}} \cdot\left(\frac{1}{2} \mathbf{r}_{i j}^{\prime}\right) \frac{\partial_{\mathbf{r}_{j}} n_{j}}{n_{j}} \cdot\left(\frac{1}{2} \mathbf{r}_{i j}^{\prime}\right)-2 \frac{\partial_{\mathbf{r}_{i}} n_{i}}{n_{i}} \cdot\left(\frac{1}{2} \mathbf{r}_{i j}\right) \frac{\partial_{\mathbf{r}_{j}} n_{j}}{n_{j}} \cdot\left(\frac{1}{2} \mathbf{r}_{i j}\right) \\
& \left.+\frac{\partial_{\mathbf{r}_{j}}^{2} n_{j}}{n_{j}}:\left(\left(\frac{1}{2} \mathbf{r}_{i j}^{\prime}\right)^{\otimes 2}-\left(\frac{1}{2} \mathbf{r}_{i j}\right)^{\otimes 2}\right)\right) \mathrm{d} \boldsymbol{c}_{i} \mathrm{~d} \mathbf{r}_{i j} \mathrm{~d} \boldsymbol{c}_{j},
\end{aligned}
$$

and both contributions vanish from the integral relations (D.12) and the hard potential approximation that yields $\delta \mathbf{r}_{i}=0$ and $\mathbf{r}_{i j}^{\prime}=\mathbf{r}_{i j}$. The remaining corrections then necessarily involve derivatives of $\widetilde{f}_{i}^{(0)}$ or $\widetilde{f}_{j}^{(0)}$ and are thus negligible when assuming (6.19) and (6.20).

Proceeding as for the energy $\mathcal{E}$, the difference $\delta^{\mathrm{Bo}} \mathcal{Q}_{1}^{\mathrm{P}}$ in the first potential part of the heat flux $\mathcal{Q}_{1}^{\text {P }}$ is obtained in the form

$$
\delta^{\mathrm{Bo}} \mathcal{Q}_{1}^{\mathrm{P}}=\sum_{i, j \in \mathfrak{S}} \int \frac{1}{4} \varphi_{i j}\left(\boldsymbol{c}_{i}-\boldsymbol{v}\right)\left(\partial_{\left(\mathbf{r}_{i}, \mathbf{r}_{j}\right)}^{2} f_{i j}^{(0), \mathrm{Bo}}:\left(\delta \mathbf{r}_{i}, \delta \mathbf{r}_{j}\right)^{\otimes 2}-\partial_{\mathbf{r}_{j}}^{2} f_{i j}^{(0), \mathrm{sy}}: \mathbf{r}_{i j}^{\otimes 2}\right) \mathrm{d} \boldsymbol{c}_{i} \mathrm{~d} \mathbf{r}_{i j} \mathrm{~d} \boldsymbol{c}_{j},
$$

and all the main contributions associated with second order density derivatives vanish since they are odd with respect to the velocities $\boldsymbol{c}_{i}-\boldsymbol{v}$ and the remaining terms may then be estimated to be negligible as for $\delta^{\text {Bo } \mathcal{E}}$ in the regime under consideration (6.19)(6.20).

Proceeding as for the pressure tensor $\mathcal{P}$, the Bogoliubov correction $\delta^{\text {Bo }} \mathcal{Q}_{2}^{\text {P }}$ in the first potential part of the heat flux $\mathcal{Q}_{2}^{\mathrm{P}}$ is obtained in the form

$$
\begin{aligned}
\delta^{\mathrm{Bo}} \mathcal{Q}_{2}^{\mathrm{P}}=-\sum_{i, j \in \mathfrak{S}} \frac{1}{8} \int \frac{\varphi_{i j}^{\prime}\left(r_{i j}\right)}{r_{i j}} \mathbf{r}_{i j} \mathbf{r}_{i j} \cdot\left(\boldsymbol{c}_{i}-\boldsymbol{v}+\boldsymbol{c}_{j}-\boldsymbol{v}\right) \\
\quad \times\left(f_{i j}^{(0), \mathrm{Bo}}-f_{i j}^{(0), \mathrm{sy}}\right)\left(\mathbf{r}_{i}^{\alpha}, \mathbf{r}_{j}^{\alpha}\right) \mathrm{d} \alpha \mathrm{d} \boldsymbol{c}_{i} \mathrm{~d} \mathbf{r}_{i j} \mathrm{~d} \boldsymbol{c}_{j},
\end{aligned}
$$


where we have denoted for convenience $\mathbf{r}_{i}^{\alpha}=\mathbf{r}_{i}-(1-\alpha) \mathbf{r}_{i j}$ and $\mathbf{r}_{j}^{\alpha}=\mathbf{r}_{i}+\alpha \mathbf{r}_{i j}$ and have left implicit the dependence of $f_{i j}^{(0), \text { Bo }}-f_{i j}^{(0), \text { sy }}$ on the velocities $\boldsymbol{c}_{i}$ and $\boldsymbol{c}_{j}$. We may thus write that

$$
\begin{gathered}
\delta^{\mathrm{Bo}} \mathcal{Q}_{2}^{\mathrm{P}}=-\sum_{i, j \in \mathfrak{S}} \int \frac{1}{8} \frac{\varphi_{i j}^{\prime}\left(r_{i j}\right)}{r_{i j}} \mathbf{r}_{i j} \cdot\left(\boldsymbol{c}_{i}-\boldsymbol{v}+\boldsymbol{c}_{j}-\boldsymbol{v}\right)\left(\partial_{\left(\mathbf{r}_{i}, \mathbf{r}_{j}\right)}^{2} f_{i j}^{(0), \mathrm{Bo}}:\left(\delta \mathbf{r}_{i}^{\alpha}, \delta \mathbf{r}_{j}^{\alpha}\right)^{\otimes 2}\right. \\
\left.-\partial_{\left(\mathbf{r}_{i}, \mathbf{r}_{j}\right)}^{2} f_{i j}^{(0), \mathrm{sy}}:\left(-\mathbf{r}_{i j}+\alpha \mathbf{r}_{i j}, \alpha \mathbf{r}_{i j}\right)^{\otimes 2}\right) \mathrm{d} \alpha \mathrm{d} \boldsymbol{c}_{i} \mathrm{~d} \mathbf{r}_{i j} \mathrm{~d} \boldsymbol{c}_{j},
\end{gathered}
$$

where $\delta \mathbf{r}_{i}^{\alpha}=\mathbf{r}_{i}^{\prime \alpha}-\mathbf{r}_{i}=\mathbf{r}_{i}^{\prime}-(1-\alpha) \mathbf{r}_{i j}^{\prime}-\mathbf{r}_{i}$ and $\delta \mathbf{r}_{j}^{\alpha}=\mathbf{r}_{j}^{\prime \alpha}-\mathbf{r}_{i}=\mathbf{r}_{i}^{\prime}+\alpha \mathbf{r}_{i j}^{\prime}-\mathbf{r}_{i}=$ $\mathbf{r}_{j}^{\prime}-(1-\alpha) \mathbf{r}_{i j}^{\prime}-\mathbf{r}_{i}$. We may now proceed as for the pressure tensor using the identity $\int_{0}^{1}(a+\alpha b)(c+\alpha d) \mathrm{d} \alpha=\left(a+\frac{1}{2} b\right)\left(c+\frac{1}{2} d\right)+\frac{1}{12} b d$ in order to integrate with respect to $\alpha$, and it is obtained that

$$
\begin{aligned}
\delta^{\mathrm{Bo}} \mathcal{Q}_{2}^{\mathrm{P}}=-\sum_{i, j \in \mathfrak{S}} \frac{1}{8} \int \frac{\varphi_{i j}^{\prime}\left(r_{i j}\right)}{r_{i j}} \mathbf{r}_{i j} \cdot\left(\boldsymbol{c}_{i}-\boldsymbol{v}+\boldsymbol{c}_{j}-\boldsymbol{v}\right) \\
\quad\left(\partial_{\left(\mathbf{r}_{i}, \mathbf{r}_{j}\right)}^{2} f_{i j}^{(0), \mathrm{Bo}}:\left(\delta \mathbf{r}_{i}-\frac{1}{2} \mathbf{r}_{i j}^{\prime}, \delta \mathbf{r}_{i}+\frac{1}{2} \mathbf{r}_{i j}^{\prime}\right)^{\otimes 2}-\partial_{\left(\mathbf{r}_{i}, \mathbf{r}_{j}\right)}^{2} f_{i j}^{(0), \mathrm{sy}}:\left(-\frac{1}{2} \mathbf{r}_{i j}, \frac{1}{2} \mathbf{r}_{i j}\right)^{\otimes 2}\right. \\
\left.\quad+\frac{1}{12} \partial_{\left(\mathbf{r}_{i}, \mathbf{r}_{j}\right)}^{2} f_{i j}^{(0), \mathrm{Bo}}:\left(\frac{1}{2} \mathbf{r}_{i j}^{\prime}, \frac{1}{2} \mathbf{r}_{i j}^{\prime}\right)^{\otimes 2}-\frac{1}{12} \partial_{\left(\mathbf{r}_{i}, \mathbf{r}_{j}\right)}^{2} f_{i j}^{(0), \mathrm{sy}}:\left(\frac{1}{2} \mathbf{r}_{i j}, \frac{1}{2} \mathbf{r}_{i j}\right)^{\otimes 2}\right) \mathrm{d} \alpha \mathrm{d} \boldsymbol{c}_{i} \mathrm{~d} \mathbf{r}_{i j} \mathrm{~d} \boldsymbol{c}_{j} .
\end{aligned}
$$

The dominant terms with respect to the density gradients then vanish being odd with respect to velocities $\boldsymbol{c}_{i}-\boldsymbol{v}$ and $\boldsymbol{c}_{j}-\boldsymbol{v}$ and the remaining part involving the gradients of $\widetilde{f}_{i}^{(0)}$ are negligible in the regime under consideration (6.19)(6.20).

\section{Acknowledgments}

This work was supported by the ANR INSIDE project, grant ANR-19-CE05-0037-02 of the French Agence Nationale de la Recherche.

\section{References}

[1] J. W. Cahn, and J. E. Hilliard, Free energy of a non uniform system I, Interfacial Free Energy, J. Chem. Phys. 28, 258 (1958).

[2] D.M. Anderson, G.B. McFadden and A.A. Wheeler, Diffuse Interface Methods in Fluid Mechanics, Ann. Rev. Fluid Mech. 30, 139 (1998).

[3] J.S. Rowlinson and B. Widom, Molecular Theory of Capillarity (Dover publications, Mineola, NY 1989, 2002).

[4] J.D. van der Waals, Thermodynamische Theorie der Capillariteit in de Onderstelling van Continue Dichtheidsverandering Verhand. Kon. Akad. V Wetensch. Amst., (1893). (in Dutch; English translation in J. Stat. Phys. 20, 197 (1979)).

[5] Y. Rocard, Equations hydrodynamiques avec termes capillaires, Théorie de la tension superficielle, J. Phys. Radium 4, 533 (1933).

[6] P.G. de Gennes, Wetting: statics and dynamics, Rev. Mod. Phys. 57, 827 (1985).

[7] P. Seppecher, Moving contact line in the Cahn-Hilliard theory, Int. J. Eng. Sci. 34, 977 (1996). 
[8] E. Bretin, S. Masnou, and E. Oudet, Phase-field approximations of the Willmore functional and flow, Numer. Math. 131, 115 (2015).

[9] P. Gaillard, V. Giovangigli and L. Matuszewski, A Diffuse Interface Lox/Hydrogen Transcritical Flame Model, Combust. Theory Model. 20, 486 (2016).

[10] H.G. Lee, J. Yang, S. Kim and J. Kim, Modeling and simulation of droplet evaporation using a modified Cahn-Hilliard equation, App. Math. Comp. 390, 125591 (2021).

[11] D. Nayigizente, S. Ducruix, and T. Schmitt, Development of an interface thickening method for the direct numerical simulation of compressible liquid-vapor flows in the framework of the second gradient theory, Phys. Fluids 33, 052119 (2021).

[12] Korteweg, D.J., Sur la Forme que Prennent les Equations du Mouvement Fluide si l'on tient Compte de Forces Capillaires Causées par les Variations de Densité Considérables mais Continues et sur la Théorie de la Capillarité dans l'Hypothèse d'une Variations Continue de la Densité, Arch. Neerl. Sci Exactes 6, 1 (1901).

[13] J.E. Dunn and J. Serrin, On the thermomechanics of interstitial working, Archive Rat. Mech. Anal. 133, 95 (1985).

[14] S. Gavrilyuk and S. Shugrin, Media with equations of state that depend on derivatives, J. Appl. Mech. Techn. Phys. 37, 179 (1996).

[15] V. Giovangigli, A kinetic derivation of diffuse interface fluid models, Phys. Rev. E 102, 012110 (2020).

[16] J. W. Cahn, and J. E. Hilliard, Free Energy of a Non Uniform System II, Thermodynamic Basis, J. Chem. Phys. 30, 1121 (1959).

[17] V.N. Starovoitov, Model of the motion of a two-component liquid with allowance of capillarity forces, J. Applied Mech. Tech. Phys. 39, 891 (1997).

[18] J. Lowengrub and L. Truskinovsky, Quasi-incompressible Cahn-Hilliard fluids and topological transitions, Proc. Roy. Soc. Lond. 454, 2617 (1997).

[19] F. Falk, Cahn-Hilliard theory and irreversible thermodynamics, J. Non-Equil. Thermo. 17, 53 (1992).

[20] M. Verschueren, A Diffuse-Interface Model for the Structure Development in Flow (PhD, Eindhoven University, 1999).

[21] M. Heida, J. Málek and K.R. Rajagopal, On the development and generalizations of Cahn-Hilliard equations within a thermodynamic framework, Z. Angew. Math. Phys. 63, 145 (2012).

[22] Z. Guo and P. Lin, A thermodynamically consistent phase-field model for two-phase flows with thermocapillarity effects, J. Fluid Mech. 766, 226 (2015).

[23] J. Liu, G. Amberg, and M. Do-Quang, Diffuse interface method dor a compressible binary fluid, Phys. Rev. E 93, 013121 (2016).

[24] H.W. Alt and I. Pawlow, A mathematical model of dynamics of non-isothermal phase separation, Phys. D 59, 389 (1992). 
[25] S. Kjelstrup and D. Bedeaux, Non-Equilibrium Thermodynamics of Heterogeneous Systems (World Scientific Pub., Singapore, 2008).

[26] J.G. Kirkwood and F.P. Buff, The statistical theory of surface tension, J. Chem. Phys. 17, 338 (1949).

[27] S. Ono and S. Kondo, Molecular theory of surface tension in liquids, in: Encyclopedia of Physics, S. Flüdge ed., 10, 134 (1960).

[28] R. Evans, The nature of the liquid-vapour interface and other topics in the statistical mechanics of non-uniform, classical fluids, Adv. Phys. 28, 143 (1979).

[29] H.T.. Davis and L.E. Scriven, Stress and structure in fluid interfaces Adv. Chem. Phys., Vol. XLIX, I. Prigogine and S. A. Rice eds, 1982.

[30] A. Frezzotti and P. Barbante, Kinetic theory aspects of non-equilibrium liquid-vapor flows, Mech. Eng. Rev. 4, 16-00540 (2017).

[31] K. Aoki, Y. Sone, and T. Yamada, Numerical analysis of gas flows condensing on its plane condensed phase on the basis of kinetic theory, Phys. Fluids A 2, 1867 (1990).

[32] C. Cercignani, Rarefied Gas Dynamics (Cambridge university press, Cambridge, 2000).

[33] A. Frezzotti, Boundary conditions at the vapor-liquid interface, Phys. Fluids 23, 030609 (2011).

[34] L. de Sobrino, On a kinetic equation of a van der Waals gas, Can. J; Phys. 45, 363 (1967).

[35] M. Grmela, Kinetic equation approach to phase transitions, J. Stat. Phys. 3, 347 (1971).

[36] J. Karkheck and G. Stell, Mean field kinetic theories, J. Chem. Phys. 75, 1475 (1981).

[37] A. Frezzotti, L. Gibelli, and S. Lorenzani, Mean field kinetic theory description of evaporation of a fluid into vacuum, Phys. Fluids 17, 012102 (2005).

[38] K. Piechór, Non-local Korteweg stresses from kinetic theory point of view, Arch. Mech. 60, 23 (2008).

[39] E.S. Benilov and M.S. Benilov, Semiphenomenological model for gas-liquid phase transition, Phys. Rev. E 93, 032148 (2016).

[40] S. Takata and T. Noguchi, A Simple Kinetic Model for the Phase Transition of the van der Waals Fluid, J. Stat. Phys. 172, 880 (2018).

[41] A. Frezzotti, L. Gibelli, D.A. Lockerby, and J.E. Sprittles, Mean field kinetic theory approach to evaporation of a binary liquid into vacuum, Phys. Rev. Fluids 3, 054001 (2018).

[42] S. Takata, T. Matsumoto, A. Hirahara, and M. Hattori, Kinetic theory for a simple modeling of a phase transition: Dynamics out of local equilibrium, Phys. Rev. E 98, 052123 (2018). 
[43] A.C. Fowler, Phase transition in the Boltzmann-Vlasov equation, J. Stat. Phys. 174, 1011 (2019).

[44] E.S. Benilov and M.S. Benilov, The Enskog-Vlasov equation: a kinetic model describing gas, liquid, and solid, J. Stat. Mech. 10, 103205 (2019).

[45] A. Frezzotti and H. Struchtrup, Grad's 13 moments approximation for the EnskogVlasov equation, AIP Conference Proceedings 2132, 120007 (2019).

[46] S. Takata, T. Matsumoto, and M. Hattori, Kinetic model for the phase transition of a van der Waals fluid, Phys. Rev. E 103, 062110 (2021).

[47] P. Barbante and A. Frezzotti, A comparison of models for the evaporation of a Lennard-Jones fluid, Eur. J. Mech. B. Fluids 64, 69 (2017).

[48] Y. Rocard, Thermodynamique (Masson \& Compagnie, Paris, 1952).

[49] D.E. Bennett and C.F. Curtiss, Density effects on the transport coefficients of gaseous mixtures, J. Chem. Phys. 51, 2811 (1969).

[50] E.G.D. Cohen, L.S. Garcia-Colin and M.H. Ernst, Kinetic theory of moderately dense gas mixtures I, Physica 50, 177 (1970).

[51] Braun, E.; Flores, A.; García-Colín, L. S., On the nonequilibrium statistical mechanics of a binary mixture. I. The distribution functions, J. Stat. Phys. 8, 239 (1973).

[52] S. T. Choh and G.E. Uhlenbeck, The kinetic Theory of Phenomena in Dense Gases (PhD dissertation, University of Michigan, 1958).

[53] F. Andrews, On the solution of the BBGKY equations for a dense classical gas, J. Math. Phys. 6, 1496 (1965).

[54] L.S. García-Colín, M.S. Green, and F. Chaos, The Chapman-Enskog solution of the generalized Boltzmann equation, Physica 32, 450 (1966).

[55] R.A. Piccirelli, Some Properties of the Long-Time Values of the Probability Densities for Moderately Dense Gases, J. Math. Phys. 7, 922 (1966).

[56] S. Chapman and T.G. Cowling, The Mathematical Theory of Non-Uniform Gases (Cambridge University Press, Cambridge, 1970).

[57] J.H. Ferziger, H.G. Kaper, Mathematical Theory of Transport Processes in Gases (North Holland, Amsterdam, 1972).

[58] J.R. Dorfman and H. van Beijeren, The kinetic theory of gases, in: Statistical Mechanics, Part B, B.J. Berne, ed., Plenum Press, New York, 65 (1977).

[59] S.R. de Groot and P. Mazur, Non-Equilibrium Thermodynamics, (Dover publications, Mineola, 1984).

[60] Giovangigli V. and Matuszewski L., Mathematical modeling of supercritical multicomponent reactive fluids. Math. Mod. Meth. App. Sci. 23, 2193 (2013).

[61] J. Keizer, Statistical Thermodynamics of Nonequilibrium Processes (Springer Verlag, New York, 1987) 
[62] V. Giovangigli, Multicomponent Flow Modeling (Birkhaüser, Boston, 1999).

[63] E. Nagnibeda and E. Kustova, Non-Equilibrium Reacting Gas Flows (Springer Verlag, Berlin, 2009).

[64] M. Capitelli, D. Bruno, and A. Laricchiuta, Fundamental Aspects of Plasma Chemical Physics, Transport (Springer, Berlin, 2013).

[65] D. Jamet, Diffuse interface models in fluid mechanics, Researchgate, (2005), https://www.researchgate.net/profile/Didier_Jamet/publication/254424182.

[66] N.N. Bogolioubov, Problems of Dynamic Theory in Statistical Physics, State Technical Press, (1946). English traduction in : Studies in Statistical Mechanics, Volume 1, J. de Boer and G.E. Ulhenbeck eds, North Holland Pub. Company, (1962), and also in Report number: AEC-TR-3852, Argonne National Laboratory, (1960).

[67] M. Born and H.S. Green, A General Kinetic Theory of Liquids, (Cambridge University press, Cambridge, 1946).

[68] J.G. Kirkwood, The Statistical Mechanical Theory of Transport Processes, J. Chem. Phys. 14, 180 (1946).

[69] J. Yvon, La Théorie Statistique des Fluides et de l'Équation d'État Actualilés Scientifiques et Industrielles, (Hermann, Paris, 1935).

[70] E.M. Lifshitz and L.P. Pitaevskii, Physical Kinetics, Landau and Lifshitz course on theoretical physics Volume 10, (Elsevier, Oxford, 1981).

[71] R.F. Snider and C.F. Curtiss, Kinetic theory of moderately dense gases, Phys. Fluids 1, 122 (1958).

[72] D.K. Hoffman and C.F. Curtiss, Kinetic theory of dense gases. III. The generalized Enskog equation, Phys. Fluids 7, 1887 (1964).

[73] R.L. Stratonovich, The entropy of a system with a random number of particles, Sov. Phys. JETP 1, 254 (1955).

[74] R.E. Nettleton and M.S. Green, Expression in terms of molecular distribution functions for the entropy density in an infinite system, J. Chem. Phys. 29, 1365 (1958).

[75] Yu.L. Klimontovich, The Boltzmann H-theorem for an imperfect gas, Soviet Phys. 36, 78 (1973).

[76] G.A. Martynov, Liouville theorem and the problem of the increase of entropy, JETP 80, 1056 (1995).

[77] A. Singer, Maximum entropy formulation of the Kirkwood superposition approximation, J. Chem. Phys. 121, 3657 (2004).

[78] V. Giovangigli and L. Matuszewski, Supercritical fluid thermodynamics from equations of state, Physica D 241, 649 (2012).

[79] M.H. Ernst, Formal theory of transport coefficients to general order in the density, Physica 32, 209 (1966). 
[80] M.H. Ernst, Kinetic theory of moderately dense gas mixtures II, Symmetry relations for transport coefficients, Physica 50, 477 (1970).

[81] V. I. Kurochkin, S. F. Makarenko, and G. A. Tirskii, Transport coefficients and the Onsager relations in the kinetic theroy of dense gas mixtures, J. Appl. Mech. Tech. Phys. 25, 218 (1984).

[82] L. Waldmann, Transporterscheinungen in Gasen von mittlerem Druck, Handbuch der Physik 12, 295 (1958).

[83] J. Van de Ree, On the definition of the diffusion coefficients in reacting gases, Physica 36, 118 (1967).

[84] R. Fowler and E.A. Guggenheim, Statistical Thermodynamics (Cambridge university press, Cambridge, 1956).

[85] L.D. Landau and E.M. Lifshitz, Statistical Physics Landau and Lifshitz course on theoretical physics, Volume 5, Elsevier, Oxford, 1980).

[86] Diu, B., C. Guthmann, D. Lederer, and B. Roulet, Éléments de Physique Statistique (Hermann, Paris, 1989).

[87] D. Berthelot, Sur le mélange des gaz, Compte Rend. Acad. Sci. 126, 1703 (1898).

[88] J. Fisher and M. Methfessel, Born-Green-Yvon approach to the local densities of a dilute fluid at interfaces, Phys. Rev. A 22, 2836 (1980).

[89] T.R. Osborn and C.A. Croxton, Monotonic and oscillatory profiles at the free liquid surface for simple atomic fluids, Mol. Phys. 40, 1489 (1980).

[90] S. Benzoni-Gavage, R. Danchin, S. Descombes, and D. Jamet, Structure of Korteweg models and stability of diffuse interfaces, Interfaces Free Bound. 7, 371 (2005).

[91] B. Haspot, Existence of strang solutions for nonisothermal Korteweg system, Annales Math. Blaise Pascal 16, 431 (2009).

[92] D. Bresch, M. Gisclon, I. Lacroix-Violet, On Navier-Stokes-Korteweg and EulerKorteweg Systems: Application to Quantum Fluids Models, Arch. Rat. Mech. and Anal. 233, 975 (2019).

[93] V. Giovangigli, Higher Order Entropies, Arch. Rat. Mech. Anal. 187, 221 (2008).

[94] V. Giovangigli, Higher Order Entropies for Compressible Fluid Models, Math. Mod. Meth. Appl. Sci. 19, 67 (2009).

[95] V. Giovangigli, Persistence of Boltzmann Entropy in Fluid Models, Disc. Cont. Dyn. Syst. 24, 95 (2009).

[96] O. Redlich and J. N. S. Kwong, On the thermodynamics of solutions. V An equation of state. Fugacities of gaseous solutions, Chem. Reviews, 44, 233 (1949).

[97] V. Giovangigli, L. Matuszewski, and F. Dupoirieux, Detailed modeling of transcritical planar $\mathrm{H}_{2}-\mathrm{O}_{2}-\mathrm{N}_{2}$ flames, Comb. Theory Mod. 15, 141 (2011). 
[98] P. Gaillard, V. Giovangigli and L. Matuszewski, Multicomponent Transport in High Pressure Flows, in High Pressure Flows for Propulsion Applications, J. Bellan ed., Progress in Astronautics and Aeronautics, 260, American Institute of Aeronautics \& Astronautics, 485 2020).

[99] A.N. Gorban and I.V. Karlin, Beyond Navier-Stokes equations: Capillary of ideal fluids, Contemp. Phys. 58, 70 (2017).

[100] A.N. Gorban and I.V. Karlin, Hilbert's sixth problem: Exact and approximate hydrodynamic manifolds for kinetic equations, Bull. Amer. Math. Soc. 51, 187 (2014).

[101] M. Slemrod, Hilbert's sixth problem and the failure of the Boltzmann to Euler limit, Phil. Trans. R. Soc. A 376, 20170222 (2018).

[102] M.S. Green, Surface integral from for three-body collision in the Boltzmann equation, Phys. Rev. 136, A905 (1964).

[103] F. Chaos and L.S. García-Colín, Density expansions of the transport coefficients for a moderately dense gas, Phys. Fluids 9, 382 (1966). 